Portland State University

PDXScholar

5-4-1992

\title{
Brainstem Auditory Evoked Potentials in Anuran Amphibians
}

Marc Brandon Carey

Portland State University

Follow this and additional works at: https://pdxscholar.library.pdx.edu/open_access_etds

Part of the Biology Commons, and the Physiology Commons Let us know how access to this document benefits you.

Recommended Citation

Carey, Marc Brandon, "Brainstem Auditory Evoked Potentials in Anuran Amphibians" (1992). Dissertations and Theses. Paper 4267.

https://doi.org/10.15760/etd.6151

This Thesis is brought to you for free and open access. It has been accepted for inclusion in Dissertations and Theses by an authorized administrator of PDXScholar. Please contact us if we can make this document more accessible: pdxscholar@pdx.edu. 
AN ABSTRACT OF THE THESIS OF Marc Brandon Carey for the Master of Science in Biology presented May 4, 1992.

Title: Brainstem Auditory Evoked Potentials in Anuran Amphibians

APPROVED BY MEMBERS OF THE THESIS COMMITTEE:

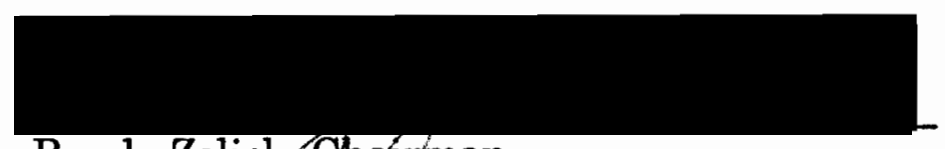

Randy Zelick,Chaírman

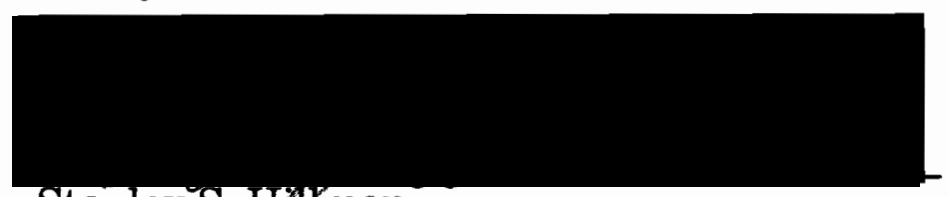

Stanley S. Hillman

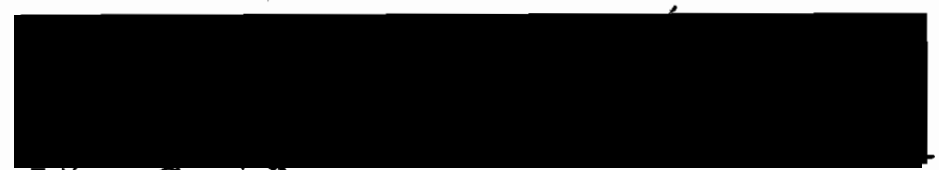

Lárry Crayshaw

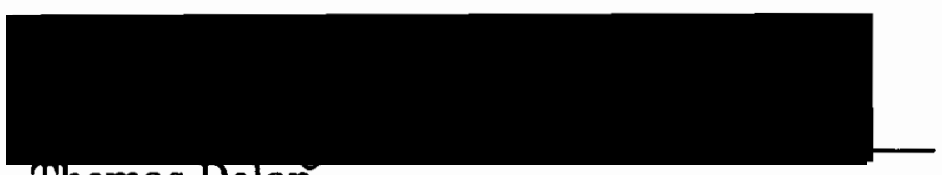

Thomas Dolan

In this study, I looked at the effects of sound level, temperature and dehydration/hypernatremia on the brainstem auditory evoked potential (BAEP) of four species of anuran amphibians (Rana pipiens, Rana catesbeiana, Bufo americanus and Bufo terrestris). The BAEP was used because it allowed me to monitor both the peripheral and central aspects of auditory nervous function simultaneously and over a long period of time. 
The BAEP was recorded using a pair of electrodes implanted in the cranial space immediately superior to the brainstem and inserted into the dorsal lymphatic sac, respectively. The acoustic stimulus was one cycle of a $1-\mathrm{kHz}$ sinusoid presented at a rate of $10 / \mathrm{s}$ at various sound levels. We averaged the BAEP over $1024(20 \mathrm{~ms})$ recorded samples.

Thresholds determined using the BAEP were comparable to those found using other neurophysiological techniques and 5-20 dB higher than those measured behaviorally. I-II interpeak latency was significantly less and BAEP threshold was significantly lower for the ranids compared to the bufonids (Mann-Whitney U test, $\mathrm{p}<.01$ ). Absolute peak latencies increased linearly while the interpeak latencies remain unchanged with decreasing sound level, which may support the theory that a traveling wave is associated with the amphibian papilla.

Decreasing temperature induced a nonlinear increase in the BAEP threshold of Rana pipiens. This nonlinear increase in BAEP threshold correlated with an increased III-IV interpeak latency. It is suggested that the increased latency is associated with an "attenuation" of the signal between peaks III and IV and results in the BAEP threshold increase. Rana pipiens acclimated to $14^{\circ} \mathrm{C}$ had a marked increase in the BAEP threshold at temperatures above $20^{\circ} \mathrm{C}$ compared with frogs acclimated to $21^{\circ} \mathrm{C}$. A decrease in the membrane fluidity of the sensory epithelium and of peripheral neurons due to a lower acclimation temperature may decrease the sensitivity of the voltage sensitive ion channels, resulting in the BAEP threshold shift.

As Rana pipiens and Bufo americanus were dehydrated to their "critical activity point" (CAP) there was no change in any of the parameters of 
the BAEP. Once CAP was reached for Rana pipiens, all of the BAEP parameters increased markedly. For Bufo americanus, only III-IV interpeak latency showed any increase once CAP was reached.

Animals were injected with $2 \mathrm{M} \mathrm{NaCl}$ to induce hypernatremia without water loss. The peripheral peaks (I and II) of the BAEP initially decreased in latency and then progressively increased. There was no change in peaks III and IV. Very few animals reached their CAP, which was probably due to the rapidity of plasma sodium concentration increase.

The BAEP is an excellent technique to study the auditory and nervous systems of anurans because of its versatility and relative ease of use. It allows monitoring over an extended period of time compared with more invasive techniques which usually have a short preparation viability. BAEP implants in anurans may last up to six weeks. 


\title{
BRAINSTEM AUDITORY EVOKED POTENTIALS \\ IN ANURAN AMPHIBIANS
}

\author{
by \\ MARC BRANDON CAREY
}

A thesis submitted in partial fulfillment of the requirements for the degree of

\section{MASTER OF SCIENCE in \\ BIOLOGY}

Portland State University 
TO THE OFFICE OF GRADUATE STUDIES:

The members of the Committee approve the thesis of Marc Brandon Carey presented May 4, 1992.

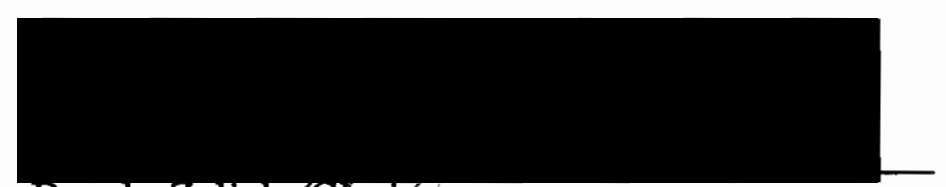

Randy Zelick,Chairman

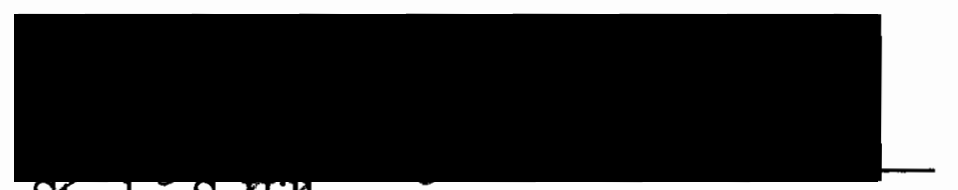

Stanley S. Ayuman

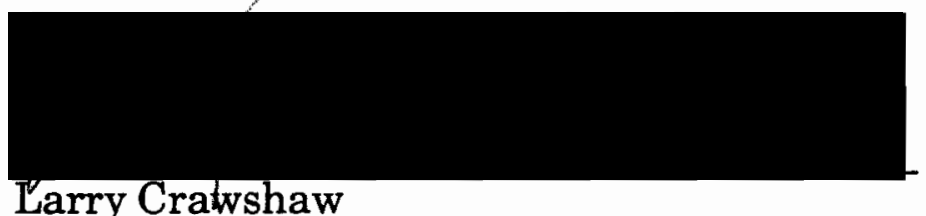

Larry Cratwshaw

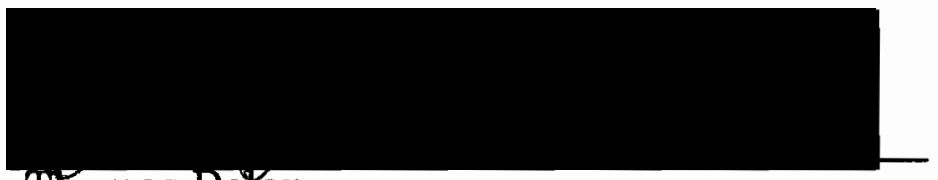

Tȟomas Dơan

\section{APPROVED:}

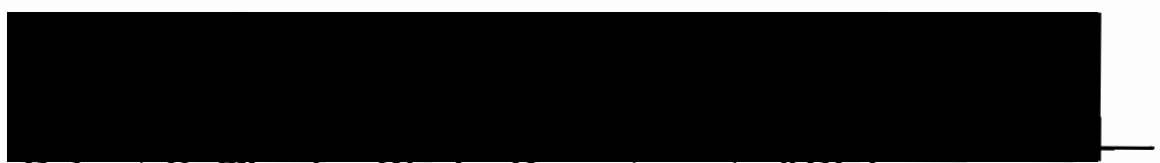

Robert U.' 'Iinnin, Chair, Department of Biology

C. William Savery, Interim Vice pyovost for Graduate Studies and Research 


\section{ACKNOWLEDGEMENTS}

I would like to thank all of the little people who helped make this project possible:

To Randy Zelick for the incredible amount of time, money and energy he infused into this project. It was through his guidance and support that I was able to get the best out of my graduate experience. May he one day find the light again and return to the little froggies. They miss him very much.

To Debbie Duffield who gave me the opportunity to enter the graduate program and show her what I could do.

To Stan Hillman for his guidance through the turbulent portion of my thesis and the use of his old squash racket.

To Larry Crawshaw for his contributions to the temperature portion of my thesis. I would also like to thank him for his tutelage of squash lore.

To Richard Forbes who made working for the sheep project a bearable experience.

To Jim Stapleton for the incredible amount of time and energy he has put into the lab and my project to make life easier.

To Zelick's Zoological Zealots who made it easy for me to complete the research for my thesis.

Most importantly, to my wife, Shannon, who gave all the love and support I needed to complete the task. None of this would be the same without her. 


\section{TABLE OF CONTENTS}

PAGE

ACKNOWLEDGEMENTS ............................................................................ ii

LIST OF TABLES......................................................................................

LIST OF FIGURES .....................................................................................

GENERAL INTRODUCTION.................................................................... ix

\section{CHAPTER}

I PARAMETERIC ANALYSIS OF THE ANURAN BRAINSTEM AUDITORY EVOKED POTENTIAL ................................................. 1

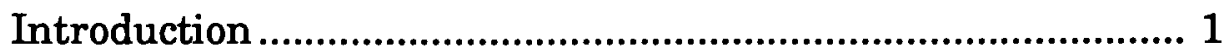

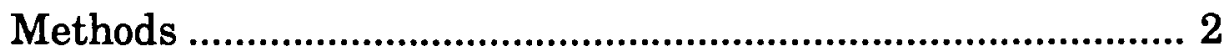

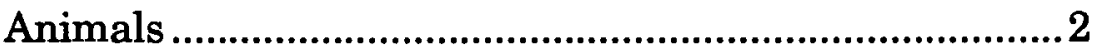

Electrode implantation ..............................................2

Stimulation and Recording ............................................ 3

Sound level and threshold.............................................. 6

Drug effects........................................................................

Data transformation and statistical analysis ..................

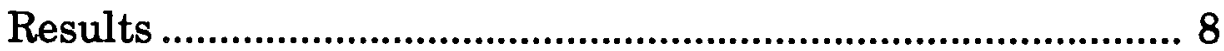

Waveform morphology ...................................................

Sound level and Threshold ............................................10

Drug effects........................................................................ 20

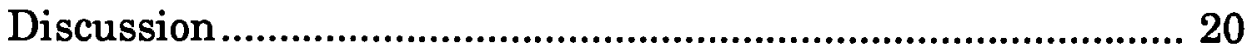

Waveform morphology ................................................20

Sound level and Threshold .............................................22

Drug effects.......................................................................27 
II EFFECTS OF TEMPERATURE ON THE ANURAN

BRAINSTEM AUDITORY EVOKED POTENTIAL...................... 29

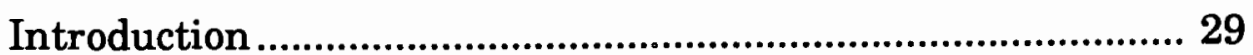

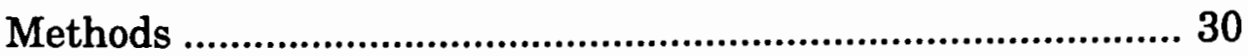

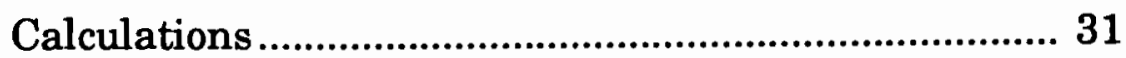

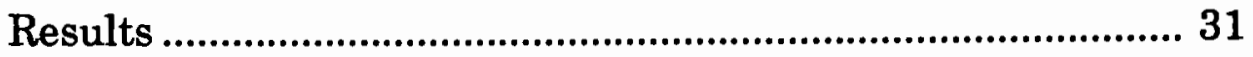

Temperature effects on the BAEP................................ 31

Effects of acclimation on the BAEP............................... 39

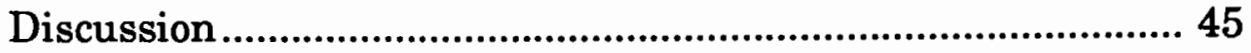

Temperature effects on the BAEP.................................45

Effects of acclimation on the BAEP...............................50

III EFFECTS OF DEHYDRATION AND HYPERNATREMIA ON THE BRAINSTEM AUDITORY EVOKED POTENTIAL OF THREE SPECIES OF ANURANS ...............................................53

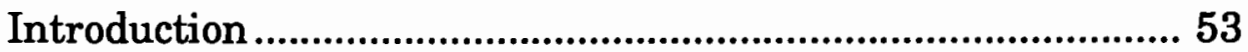

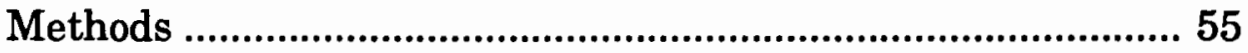

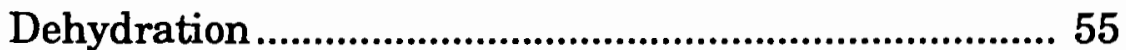

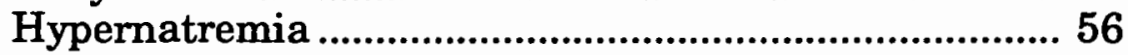

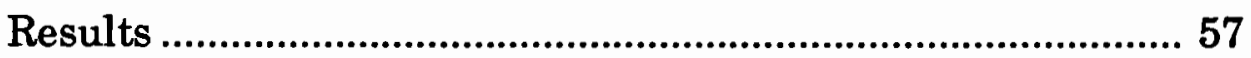

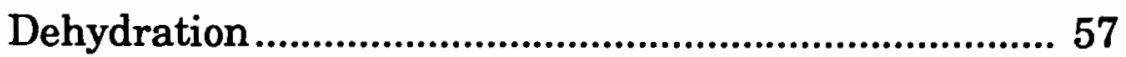

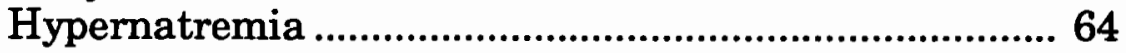

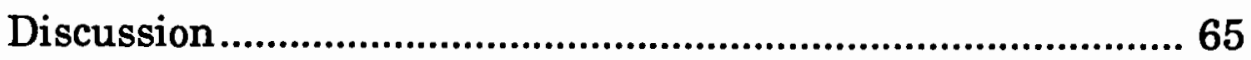

IV GENERAL DISCUSSION ...................................................6

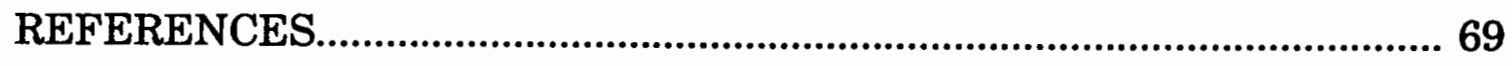




\section{LIST OF TABLES}

TABLE

PAGE

I Mean ( $\mathrm{n}=5$, per specie) signal-to-noise ratios with standard deviations for three species of anurans at different test stimulus levels (dB SPL) ..................................... 12

II Averages and standard deviation (s.d.) for selected parameters of the brainstem auditory evoked potential in Rana pipiens $(n=4)$ 14 


\section{LIST OF FIGURES}

FIGURE

PAGE

1

2

3

4

5

6

7

8

9

10

11

12

Stimulus waveform used ........................................................... 4

Equipment arrangement for obtaining frog BAEPS ................5

Typical BAEPs for three species of anurans............................9

Typical BAEP change in response to stimulus level $(\mathrm{dB}$

SPL)

Distributions of BAEP thresholds for two species of Rana

and Bufo

Absolute peak latencies versus sound level for four BAEP

peaks in three species of anurans

Interpeak latencies versus sound level for three BAEP

interpeaks in three species of anurans

A comparison of the peak I and IV amplitudes for $R$.

pipiens, $B$. americanus and $B$. terrestris versus sound

level

Effects of TMS anesthetic on the BAEP

BAEP change with temperature

BAEP threshold response to changes in temperature 34

Comparison of changes in BAEP threshold, peak I

latency, and three interpeak latencies with temperature 35

Temperature dependence of BAEP threshold shift

compared with divergence of interpeak latencies 
14 Peak I (A) and IV (B) amplitudes for R. pipiens in response to changes in temperature 38

15 BAEP threshold response vs. temperature for frogs acclimated to 14 and $21^{\circ} \mathrm{C}$ 40

16 Peak I and interpeak (I-II, II-III and III-IV) latency change to an increase in temperature for $R$. pipiens acclimated to 14 and $21^{\circ} \mathrm{C}$ 42 A comparison of the average expected BAEP threshold shift calculated from the peak I latency difference between $R$. pipiens acclimated to 14 and $21^{\circ} \mathrm{C}$ and the average BAEP threshold difference for the same frogs at six different ranges of test temperatures. Peak I (A) and IV (B) amplitudes to change in temperature for $R$. pipiens acclimated to 14 and $21^{\circ} \mathrm{C}$ 46 Effect of dehydration on the BAEP of one Rana pipiens 58 Effect of dehydration on the BAEP of one Bufo americanus 59 Mean BAEP threshold and 95\% confidence intervals in response to changes in dehydration percentage for Rana pipiens (A) and Bufo americanus (B). 61 A comparison of the responses of the peak I latency and the I-II, II-III and III-IV interpeak latencies to increased water loss for Rana pipiens and Bufo americanus 62 A comparison of the peak I (A) and IV (B) mean amplitudes for Rana pipiens and Bufo americanus to increases in the dehydration percentage 


\section{GENERAL INTRODUCTION}

\section{ANURAN AUDITORY SYSTEM}

Numerous facets of the anuran (frog and toad) auditory system have been researched, ranging from behavioral to physiological studies. The anuran auditory system is excellent for auditory research, since it is simple relative to the mammalian cochlea yet very highly adapted to detect, in many cases, complex conspecific vocalizations essential for social interactions. Furthermore, when compared with other vertebrates, anurans possess many methodological conveniences which simplify experimentation.

The ability of the anuran auditory system to process complex sounds allows for elaborate acoustic communication. The basic unit of acoustic communication for these animals is the call. There are five to seven different categories of calls identified, each of which has a specific purpose. The most common are the advertisement, territorial, release (to prevent amplexus or mating) and warning (Blair, 1958; Schiotz, 1973; Straughan, 1973) calls. Each of the call types may be distinguished within a species by differences in spectral composition and/or amplitude modulation characteristics such as pulse number and pulse repetition rate (Brzoska, et al., 1977).

Of the different types of calls used by anurans, the mating call is the most studied because it is usually the most conspicuous and has significance as a species isolating mechanism (Blair, 1958; Gerhardt, 1974; Gerhardt and Doherty, 1988; Wilczynski, et al., 1984). Recognition of species-specific mating calls is necessary for the reproductive success of the anuran 
populations. For example, Gerhardt (1974) found that frequency composition of the mating call in the green tree frog (Hyla cinerea) is the most important factor for the attraction of females of the same species. Gerhardt analyzed the mating call and found that it had a bimodal frequency spectrum with modes at $900-1100 \mathrm{~Hz}$ and $2700-3300 \mathrm{~Hz}$. A synthetic mating call composed of only two tones centered at the same frequencies as the spectral modes of the natural mating call is as attractive to female green treefrogs as the natural call. Any spectral variation in the synthetic call is less effective at attracting females.

For species that are closely related and sympatric, mating call recognition is crucial in maintaining reproductive isolation to prevent the production of non-viable offspring. Gerhardt and Mudry (1980) (Gerhardt and Mudry, 1980)describe a sympatric situation for the treefrogs, Hyla cinerea and Hyla gratiosa. Female $H$. cinerea prefer mating calls produced by conspecific males over those calls produced by male $H$. gratiosa, but it is interesting that this selectivity is not stable with temperature. At temperatures below $20^{\circ} \mathrm{C}$, the female $H$. cinerea instead prefer calls produced by the male $H$. gratiosa. The reason for this change in mating call preference is that the best excitatory frequency for the low-frequency portion of the bimodal audiogram for female $H$. cinerea decreases from approximately $900 \mathrm{~Hz}$ to $600 \mathrm{~Hz}$ at cooler temperatures, which is closer to the frequency spectrum peak of the mating call produced by the male $H$. gratiosa. A similar circumstance has been described in the sympatric species $H$. versicolor and $H$. chrysoscelis (Rose, et al., 1985).

The anuran inner ear is relatively simple compared to the mammalian inner ear. Unlike most mammals, anurans lack an outer ear, and the 
tympanic membrane is flush with the external surface of the animal. The middle ear cavity is present in the anuran, but the method by which the acoustic stimulus is transduced from the tympanic membrane to the oval window of the inner ear cavity is not the same as in mammals. Sound is conducted via three pathways in the mammalian middle ear: (1) bone conduction, (2) air in the middle ear cavity, and (3) across the middle ear cavity by way of the ossicular chain (malleus, incus and stapes) (for a recent review see Yost and Nielsen, 1985). Two major pathways for sound conduction have been extensively studied in the anurans: (1) the opercular complex and (2) the columella and tympanum (Lombard and Straughan, 1974). The opercular complex is comprised of the opercular muscle which arises from the anterodorsal surface of the suprascapula and inserts on the dorsal surface of the operculum (Wever, 1985). Low frequency sounds are received by the forelimbs of the anurans and are propagated along the opercular muscle and transferred to the operculum of the inner ear cavity. High frequency sounds are received by the tympanum. The mechanical vibration of the tympanic membrane is transmitted by the columella to the oval window of the inner ear cavity.

The generic function of the components in the middle ear cavities of all animals is to amplify the sound pressure delivered from the tympanic membrane to the oval window (Yost and Nielsen, 1985). This amplification is necessary to offset the attenuation (about $30 \mathrm{~dB}$ ) of the acoustic signal due to the impedance difference between the air and the fluid in the inner ear. In mammals, the arrangement of the three middle ear bones and the area ratio of the tympanum to oval window largely compensate for the impedance difference. The area at the head of the malleus, which is attached to the 
tympanic membrane, is $\mathbf{1 7}$ times greater than the area at the stapes

footplate, which is attached to the oval window. The difference in the areas of the tympanum and oval window corresponds to a 17 times greater sound pressure difference. The three bones are arranged such that they work as a lever system, which further increases the pressure 1.3 times. The total pressure increase is 22 times the initial sound pressure level, which corresponds to only a loss of $3 \mathrm{~dB}$ due to the impedance difference (Yost and Nielsen, 1985).

The anuran middle ear has only one middle ear bone, the columella, which acts to transduce the mechanical energy from the tympanic membrane to the oval window. There is a similar area differential between the tympanum and oval window, but the columella does not act as a lever (Wever, 1985). This results in a smaller amplification of the acoustic stimulus as compared to the mammalian condition and is part of the reason anurans are less sensitive to sound than most mammals.

There are even larger differences between mammals and frogs when considering the inner ear. The frog ear, both left and right, contains two separate auditory endorgans, the amphibian and basilar papillae.

The amphibian papilla in the anuran inner ear is analogous to the mammalian cochlea. As in the mammalian cochlea, the amphibian papilla is tonopically organized with the hair cells sensitive to the high frequency stimuli located on the basal portions and the low frequency sensitive hair cells located on the apical portions of the papilla (Lewis, 1984). The human cochlea has range of sensitivity from $6 \mathrm{~Hz}$ to $>50 \mathrm{kHz}$ (Bekesy, 1960; Corso, 1963), while the amphibian papilla is most sensitive to frequencies ranging from 100 to $1500 \mathrm{~Hz}$ (Feng, et al., 1975; Lewis, et al., 1982b). The 
mammalian cochlea uses a traveling wave mechanism, which is supported by the basilar membrane, to resolve complex acoustic signals into their spectral components. There has been no direct evidence for the presence of a traveling wave in the amphibian papilla, however, there has been some indirect evidence using neurophysiological techniques. The tectorial membrane, which overlies the sensory epithelium of the amphibian papillae, may be the structure which supports the traveling wave (Hillery and Narins, 1984).

The basilar papilla is a much simpler auditory endorgan than the amphibian papilla and it is most sensitive to a very narrow frequency range, above $1000 \mathrm{~Hz}$ (Wilczynski, et al., 1984). Although the range of frequency sensitivity for the amphibian papilla is similar for most species of anurans, the frequency range for the basilar papilla differs among species (Feng, et al., 1975; Lewis, et al., 1982a) and may even different between the sexes of the same specie (Wilczynski, et al., 1984). It has been found that the range of frequency sensitivity of the basilar papilla often coincides with at least one of the dominant spectral components of the mating call (Frishkopf, et al., 1968). In the spring peeper, which has only a single spectral peak in its advertisement call, the dominant frequency is found to be centered in the range of most sensitivity for the basilar papilla of the female (Wilczynski, et al., 1984).

Once the mechanical signal is transduced to modulations of neurotransmitter by the auditory endorgans (amphibian and basilar papillae), the VIIIth cranial nerve communicates spike rate patterns to the Dorsal Medullary Nucleus (DMN) (van Stokkum and Gielen, 1989; Zakon, 1983). There are three distinct VIIIth nerve fiber types which arise from the 
auditory portion of the inner ear (Feng, et al., 1975). The different types are distinguished based on their frequency sensitivity and on the presence or absence of two-tone inhibition. Frequency sensitivity is measured typically, by generating a frequency-threshold curve, or tuning curve, which plots the neural excitation threshold as a function of stimulus tone-burst frequency. Two-tone inhibition is the suppression of the response of one single-unit (a single neuron of a nerve bundle) to an excitatory tone by the simultaneous addition of a second tone of lower or higher frequency and appropriate intensity, outside the initial single-unit's excitatory tuning curve (Feng, et al., 1975). The three types of fibers are: low-frequency-sensitive with two-tone inhibition, mid-frequency-sensitive with no two-tone inhibition and highfrequency-sensitive with no two-tone inhibition. The high-frequencysensitive fibers are thought to arise from the basilar papilla and the other two types from the amphibian papilla.

The VIIIth cranial nerve inserts at the level of the brain stem on the $\mathrm{DMN}$, carrying both vestibular and auditory information. The DMN acts as a relay to higher brain centers but is also involved in the neural processing of the behaviorally important temporal attributes of the acoustic stimulus (Hall and Feng, 1991).

The outputs of the DMN are numerous (Wilczynski and Capranica, 1984). There is a strong commissural projection to the contralateral DMN, as well as projections to the contralateral and ipsilateral torus semicircularis. The main projection of the DMN is bilateral to the superior olivary nucleus (SON).

The SON is the last nucleus of the ascending auditory pathway in the brainstem. Unlike the mammalian brain, the anuran SON is not divisible 
into the medial and lateral nuclei. There is increased binaural interaction at this level with one third of the neurons exhibiting two-tone suppression. Unlike the neurons in the VIIIth nerve, those single-units which show suppression are not only tuned to the frog's low frequency range, but to the entire range of sensitivity (Wilczynski and Capranica, 1984).

The SON sends bilateral projections to a midbrain laminated structure, torus semicircularis (TS). It is at the level of the TS that we see the first central nervous system evidence of tonotopicity. High frequency responses predominate in the rostral TS and low frequency responses in the caudal TS (Wilczynski and Capranica, 1984). Above the TS, the afferent pathway becomes more complex, but further projections will not be discussed since the focus of this thesis centers on the brainstem nuclei.

The anatomy and physiology of the anuran auditory system is relatively simple, which makes it easier to study than reptilean or mammalian models. The fact that the anurans are strongly dependent on acoustic communication in their behavioral ecology has allowed us to extensively study the anuran auditory system and to create models to better understand acoustic communication for all animals.

\section{BRAINSTEM AUDITORY EVOKED POTENTIALS}

Brainstem auditory evoked potentials (BAEPs) are microvolt potentials recorded from electrodes located near the active tissue in response to a periodic click or tone burst. In this study, BAEPs will refer to the short latency potentials which are within $10 \mathrm{~ms}$ of an acoustic stimulus onset. Successive recorded 10-ms potentials were averaged over a number of click presentations to cancel random potentials and improve signal-to-noise ratio. 
I will refer to the brainstem auditory evoked potential (BAEP) as a singular potential for convenience, although in all cases each BAEP is the average of voltage records obtained following multiple rapidly successive stimulus presentations. The BAEP is comprised of a series of peaks which are sequential in time and correspond to synchronous neural activity at different levels of the auditory pathway (Jacobson, 1985)

To elicit and record a BAEP, it is necessary to provide a proper acoustic stimulus. There are several important parameters for creating the proper acoustic stimulus: Waveform shape, stimulus rate, duration, rise/fall time, and the amplitude spectrum of the stimulus (Gorga, et al., 1985).

The two most commonly used temporal waveforms are clicks and tone bursts. Acoustic clicks are uni- or bi-directional pulses (compression and/or rarefaction) sudden onsets and decays. The frequency spectrum for a click is broad and the spectral range is determined by the duration of the click. Tone bursts are more complex. They are usually created using a waveform such as a pure tone and an envelope to control the amplitude of the tone. A pure tone is gated in bursts based with critical parameters being the rise/fall time and duration of the envelope.

There are two factors which determine the proper stimulus to use in achieving synchronous firing of neural generators and to elicit a frequency specific response (Durrant, 1988). To receive the best synchronous firing of the neural generators, the rise time of the stimulus must be as brief as possible. On one hand, generation of brief signals is limited by the bandpass characteristics of real-world acoustic drivers. On the other hand, were it possible to achieve a true delta function (instantaneous rise/fall time) the resulting spectrum would be very broad. Depending on the purpose of the 
BAEP measurements, it necessary to compromise between rise/fall time and spectral purity to achieve the best results.

The polarity of the acoustic stimulus may have an effect on the BAEP. The electrical polarity of the initial rising edge of the stimulus waveform specifies whether the acoustic transducer produces an initial condensational or rarefactional pressure wave. A rarefactional (negative) pressure will cause the tympanum to be drawn away from the middle ear cavity while condensation (positive pressure) will drive it in. For humans, ring doves and catfish, a negative polarity stimulus may elicit an increased latency for the BAEP peaks relative to a positive polarity stimulus (Borg and Lofqvist, 1982; Corwin, et al., 1982). To correct for the polarity induced differences and to cancel cochlear microphonic and artifacts, clinical BAEP testing is conducted by alternating the polarity of the acoustic stimulus.

The final stimulus parameter is the stimulus rate. The effect of stimulus rate on the BAEP has been thoroughly studied in the mammals (Don, et al., 1977; Freeman, et al., 1991; van Olphen, et al., 1979). In general, if the stimulus rate is increased above $20 \mathrm{clicks} / \mathrm{s}$, then the early components of the BAEP are diminished in amplitude and the latencies to the various peaks are increased. The recommended delivery rate for the greatest resolution is under 12/s (Schwartz and Berry, 1985).

$\mathrm{BAEPs}$ are small relative to other electrical activity throughout the body. To be able to analyze the BAEP, it is necessary to reduce the signal-tonoise ratio between the wanted potential and the ambient electrical activity as much as possible. This reduction is accomplished in five ways: (1) bandpass filtering the recorded signal, (2) artifact rejection, (3) electrode 
placement, (4) common-mode rejection, and (5) signal averaging (Schwartz and Berry, 1985).

The filter range for the bandpass filtering is based on the frequency range for the BAEP. The lower limit usually set to $100 \mathrm{~Hz}$ to filter out the 60 $\mathrm{Hz}$ noise from the $\mathrm{AC}$ power lines and the upper limit is set to twice the highest frequency of interest, typically $3000 \mathrm{~Hz}$ (Glattke, 1983).

There are two important considerations when placing the electrodes; electrode-type and impedances. When a BAEP recording is made, there typically are three electrodes used; active, reference, and ground. The active electrode is placed near or within the active neural tissue. The reference electrode is placed in the body/animal where little or no relevant activity is expected. The ground electrode is coupled, ultimately, to a true earth ground point (Glattke, 1983). Impedance is the effective resistance between the electrode surface and its contact. For BAEP recording, it is necessary to minimize the impedance between the electrodes and the subject and to match the impedances between the active and reference electrodes. The amount of impedance has a direct effect on the amount of noise recorded. If the impedances of the active and reference electrodes are mismatched, there will be a difference in the amplitude of the noise between the electrodes and this difference will be increased by the differential amplifier (Glattke, 1983).

The initial amplification of the BAEP is completed by a differential amplifier. The electrodes are connected to the differential amplifier based on their placement, inverting (reference), noninverting (active) and common (ground) inputs. Electrical signals which are similarly recorded from the active and reference electrodes (e. g. $60 \mathrm{~Hz}$ noise) will cancel before leaving the amplifier, due to the inversion and addition of the reference signal to the 
active signal. This is common-mode rejection (Glattke, 1983). The singleended output of the differential amplifier is then routed to a signal averager.

The most powerful tool to increase the signal-to-noise ratio for BAEP detection is computer-aided signal averaging. Signal averaging is the summation, and division of a number of recording samples by the number of samples, which diminishes electrode voltage fluctuations which occur randomly relative to the time of the stimulus. For my experiments an artifact reject device was additionally placed in the circuit to reject any signal larger than a prescribed amplitude. This prevents large spurious signals like muscle potentials from skewing the averaged response. The number of recordings necessary to generate a BAEP depends on the electrode position. An increase in the number of the recordings is necessary if the electrodes are further removed from the active tissue, such as scalp recordings used in human BAEP work. For best results in humans, at least 2000 samples are recommended (Schwartz and Berry, 1985). For anurans, only 1024 samples were required for a BAEP recording, since the impedance between the electrodes and the active tissue was presumably less for the anuran due to electrode position.

The recovered BAEP is made up of a series of peaks which correspond to synchronous neural generators in the peripheral and lower portions of the central nervous system. Many studies have been performed on cats and humans to determine which nuclei are responsible for the various peaks (Achor and Starr, 1980; Moller, et al., 1982; Moore, 1987). For the human BAEP, seven peaks have been identified and those nuclei responsible for their presence determined. Peak I exclusively originates from the distal part of the VIIIth nerve. Peak II originates mostly from the distal parts of the VIIIth 
nerve. Peak III is mainly generated by the cochlear nucleus. The neural generator for peak IV is primarily the superior olivary complex, with activity from the cochlear nucleus and lateral lemniscus. Peaks V, VI, and VII all seem to arise from the inferior colliculus. It is important to note that with peaks IV and beyond, the neural generators responsible for the peak production are complex and more than one neural generator is probably involved in the formation of the peak (Moller and Janetta, 1985).

Complete analysis of the BAEP is accomplished by measuring four parameters: the absolute peak latencies, interpeak latencies, amplitudes of the various peaks, and the overall waveform morphology of the BAEP (Jacobson, 1985).

The absolute latency of a peak in a BAEP is the most constant measurement of the four parameters used to analyze BAEPs. Absolute peak latency is a reflection of the conduction time between the arrival of the acoustic stimulus and the peak of interest (Durrant, 1988). Some factors which may have an impact on the absolute latency of a peak in the BAEP are nerve conduction velocity, synaptic transmission and traveling wave time in the inner ear.

The interpeak latency is the difference in absolute latency between two peaks (Durrant, 1988). Interpeak latency is a measurement of the conduction time from one neural generator to another. This is a helpful measurement to localize which portions of the neural pathway are involved in causing discovered aberrations in the BAEP.

The amplitude of a BAEP waveform is variable at best and is difficult to draw conclusions from, although general trends may be described. There are three methods for determining peak amplitude: measuring from baseline 
to peak, peak to peak (from positive to negative), and area under a peak. The first two measurements are the easiest but are not accurate descriptions for the real peak amplitude. Measurement of the area under a peak would in theory be the most accurate, but in practice it is difficult to determine the onset and termination of a peak. The ratio of peak $\mathrm{I}$ and $\mathrm{V}$ amplitudes are often used in clinical diagnosis in humans, since the ratio is relatively constant for normal BAEPs (Schwartz and Berry, 1985).

Waveform morphology is a qualitative analysis and is used for a variety of purposes. Based on the experience of an individual researcher, waveform morphology may provide intuitive information for further analysis (Schwartz and Berry, 1985).

The BAEP may be used in a number of ways to answer questions about nervous system and auditory function. Physiological stresses which may have an effect on the nervous system can be monitored using BAEPs. In the diagnosis of audiological disorders, the BAEP may be used to localize aberrations in the auditory pathway based on changes in peak amplitude and latency.

An important feature of the BAEP is that the electrodes may be noninvasive or can be implanted in such a way that it is not harmful to the animal. Unlike other neurophysiological techniques, recordings may be made over a number of days without detriment to the animal. This is very powerful, especially for those studies which may require that experimental conditions be maintained over an extended period of time to observe successive change in the nervous or auditory systems.

BAEPs may also be used to estimate auditory thresholds in those organisms which are incapable of behaviorally responding to an acoustic 
stimulus (Elberling and Don, 1987; Lasky and Yang, 1986; Sohmer, et al., 1989). Thresholds predicted by BAEP analysis, as in most neurophysiological determinations, are higher than those determined behaviorally.

Most of the BAEP research has been focused on mammals. Recently, there have been studies which have concentrated on understanding the BAEPs in non-mammalian vertebrates (Corwin, et al., 1982; Seaman, 1991; Strain, et al., 1987). One study created an audiogram for the seagull using BAEPs and also found that masking with white noise obliterates the BAEP (Counter, 1985).

The purpose of this thesis is to use the BAEP technique so that we may further understand the auditory and nervous systems of the anuran amphibian. The first section will be focused on establishing the normal parameters for the latencies and amplitudes of the various peaks in response to changes in acoustic stimulus intensities and BAEP threshold. The second section will concentrate on the effects of temperature and temperature acclimation on the BAEP. The final section will be centered on the effects of dehydration and hypernatremia on the BAEP. 


\section{CHAPTER I}

\section{PARAMETERIC ANALYSIS OF THE ANURAN BRAINSTEM AUDITORY EVOKED POTENTIAL}

\section{INTRODUCTION}

The auditory nervous system has been extensively studied in the anurans (Capranica, 1976; Pettigrew, et al., 1981; Wilczynski and Capranica, 1984). Most of these studies used either single- or multi-unit recordings of specific nerve fibers or nuclei along the auditory pathway.

Corwin, Bullock and Schweitzer (1982) were the first to describe brainstem auditory evoked potentials (BAEPs) in anurans. The purpose of their study was to create new methods of research in the non-mammalian vertebrates to answer questions which could not be answered by conventional methods. Unfortunately, since then there has been little use of the BAEPs in the anurans. Finally Seaman (1991) used BAEPs in a limited fashion to provide more information about VIIIth nerve activity in the frog, Rana catesbeiana.

Frogs and toads are not readily trained thus it has been difficult to obtain behavioral measures of auditory threshold. Some success has been obtained in one species, Rana catesbeiana, (Megela-Simmons, 1988; MegelaSimmons, et al., 1985), where behavioral threshold was determined to be about $10 \mathrm{~dB}$ lower than those determined with neurophysiological techniques 
(Penna, et al., 1990; Zelick and Narins, 1985). There has been limited use of the BAEP in determining threshold in anurans (Corwin, et al., 1982).

The purpose of the present study is to explore the BAEP in further detail for three species of anurans, Bufo terrestris, Bufo americanus and Rana pipiens. I will compare changes in all BAEP parameters in response to stimulus sound level and examine the factors influencing BAEP threshold. Finally, I will look at the effects of two drugs, d-tubocurarine chloride and tricaine methanosulfonate (TMS) on the anuran BAEP. The d-tubocurarine chloride is a paralytic agent while TMS is used as a general anesthetic for fish and anurans.

\section{METHODS}

\section{Animals}

Rana pipiens, Bufo americanus and Bufo terrestris (mean mass \pm standard deviation (s.d.) $=28.3 \pm 5.7,30.9 \pm 3.5$, and $26.9 \pm 5.5$, respectively) were purchased from commercial suppliers. Juvenile Rana catesbeiana, used for BAEP threshold analysis, were collected locally. All species were given liberal access to water and were fed Tenebria larvae once per week, except during the week prior to experimentation.

\section{Electrode implantation}

Before electrode implantation, each frog was anesthetized (Tricaine methanosulfonate [TMS], $\approx 3 \mathrm{mM}$ ), then placed dorsal side up on a layer of ice, to provide additional anesthesia and to slow blood flow. An electrode pair consisted of a 30 and a $40 \mathrm{~mm}$ length of seven-stranded stainless steel wire (diameter $=.005 \mathrm{~cm})$ coated with teflon (diameter $=.018 \mathrm{~cm} ;$ A-M Systems, 
7935). One wire (active electrode; $30 \mathrm{~mm}$ ) was positioned in the animal's cranium, via a $1 \mathrm{~mm}$ hole drilled in the dorsal aspect of the frontoparietal bone. The second wire (reference electrode; $40 \mathrm{~mm}$ ) was inserted caudally into the dorsal lymphatic sac. The inserted end of each wire had $1 \mathrm{~mm}$ of the teflon coating removed.

Both electrodes of a pair were fixed to the bony surface of the cranium with cyanoacrylate adhesive (Nexaband Opthalmic; Tri-point medical or Histoacryl blau; B. Braun Melsungen AG) and an additional covering of dental cement.

The animals were allowed 48 hours post surgical recovery before recordings were attempted.

\section{Stimulation and Recording}

All tests were performed in a double-walled sound isolated chamber (IAC 1200 series). The acoustic stimulus presented to the animal was a single-cycle of a 1-kHz sinusoid (Krohn-Hite 2100) at a repetition rate of 10/sec (Figure 1). This rate was found to be optimal for resolution of early BAEP peaks. The frequency spectrum of the acoustic stimulus was sufficient to stimulate both the amphibian and basilar papillae given their frequency of tuning in the species used (Wever, 1985). The stimulus was manually stepattenuated (HP 350B) and current boosted (NAD 3020A amplifier), to drive a free-field loudspeaker (Radio Shack Minimus 7.0). The speaker was hung from the sound chamber ceiling $0.5 \mathrm{~m}$ directly above a recording arena (for stimulus and recording equipment arrangement, see Figure 2). The overhead speaker arrangement stimulated both tympanic membranes equally regardless of the frog's orientation in the cage. The arena consisted of a plywood chamber $(81 \times 80 \times 73 \mathrm{~cm})$, the inner surface of which 


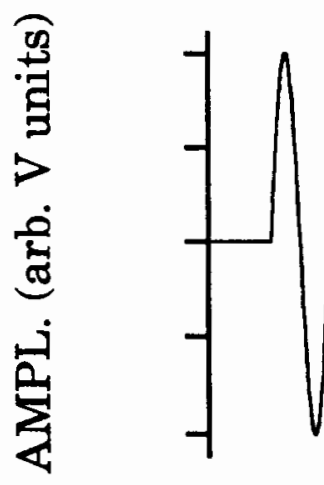

$\mathbf{A}$
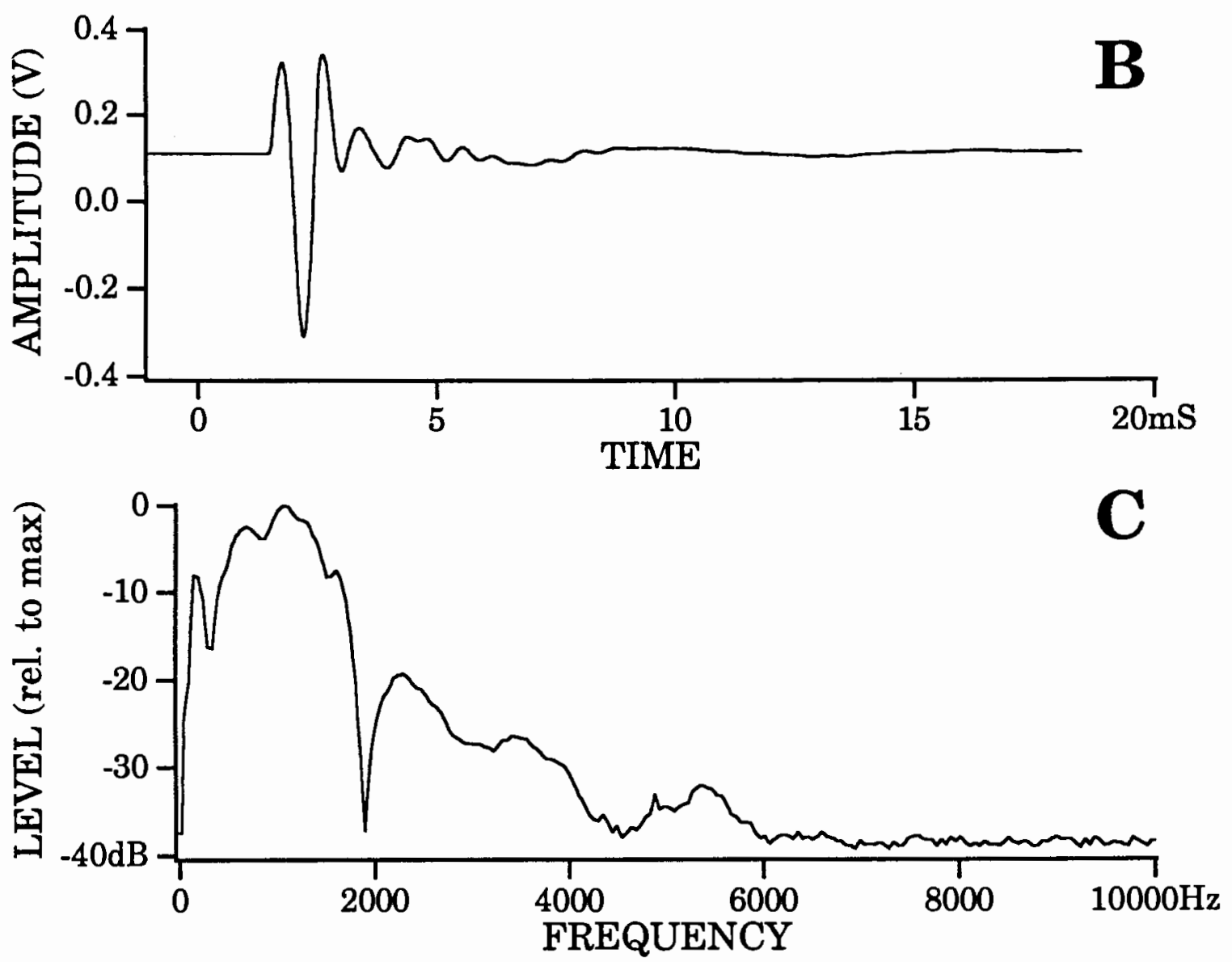

Figure 1. Stimulus waveform used. (A) Electrical signal delivered to loudspeaker. (B) Resulting acoustic signal received by pressure microphone at position of animal's ear. (C) FFT of the microphone signal. The spectrum of the acoustic signal was appropriate to stimulate both amphibian and basilar papillae VIIIn neurons. 


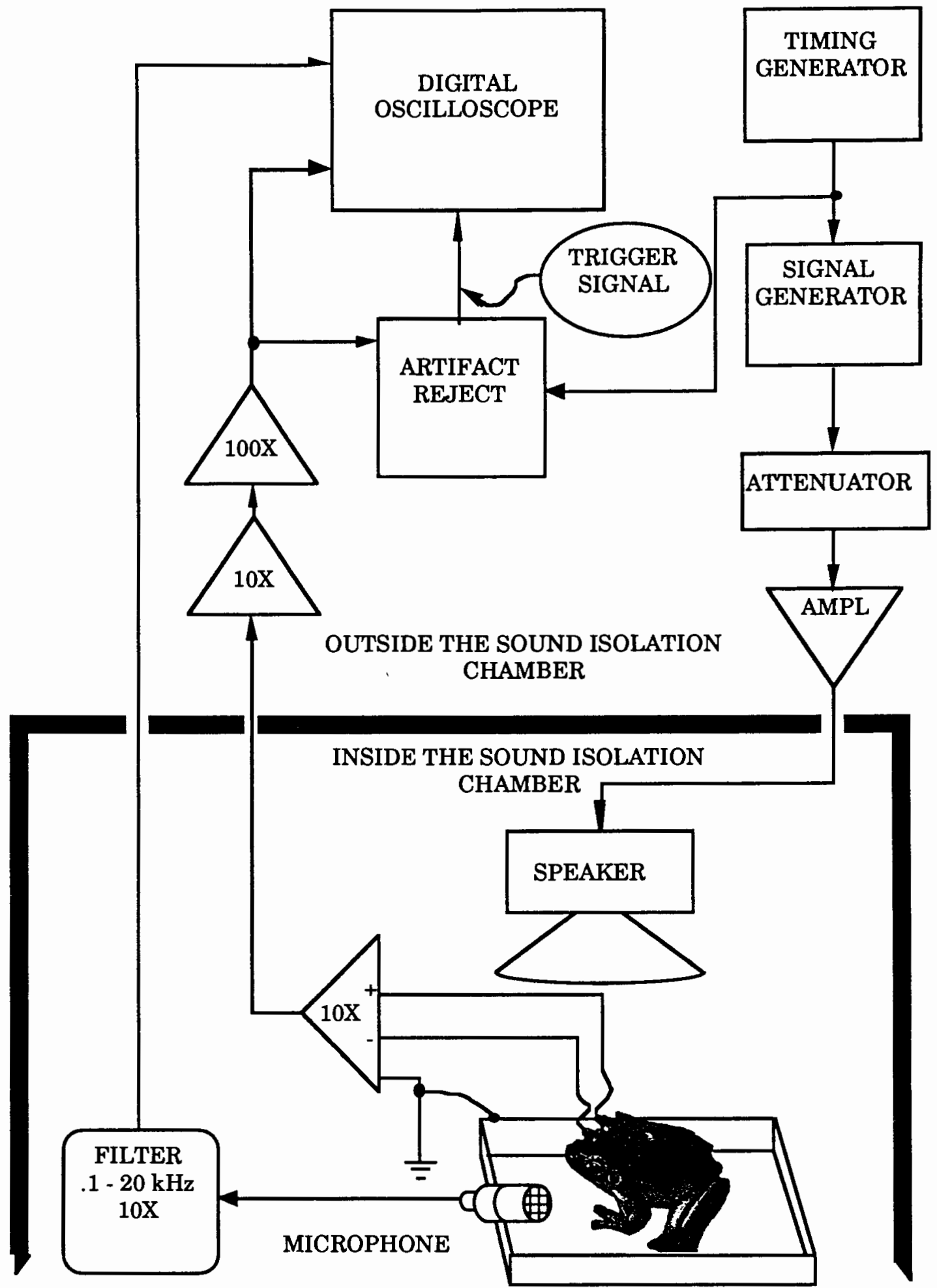

Figure 2. Equipment arrangement for obtaining frog BAEPs. See text for additional description. 
was filled with $10 \mathrm{~cm}$ of fiberglass insulation material (held in place with hardware cloth; $2.5 \mathrm{~cm}^{2}$ wire grid) to prevent acoustic reflection. A separate hardware cloth platform $73.5 \mathrm{~cm}$ from the floor supported a copper screen cage for the test animal.

The active versus reference electrode signal was differentially amplified (Grass P15; recording bandwidth: $100 \mathrm{~Hz}-3 \mathrm{kHz} ; 10 \mathrm{x}$ gain) and then single-end amplified with two additional AC amplifiers (HP 465A at 20 $\mathrm{dB}$ and $40 \mathrm{~dB}$ gain, respectively). The amplified signal was averaged using a digital oscilloscope and stored for subsequent analysis (1024 sweeps; Tek DSA601A, 14 bit equivalent amplitude precision). The oscilloscope was triggered on each stimulus (single-period sinusoid) but only if the brain electrode signal was below a threshold level. The threshold level was set to pass typical brain signals but fail on much larger muscle-generated artifacts produced if the animal moved. This artifact rejection was performed by a custom-built circuit.

During testing, sound level was monitored at the position of the frog (1/2" measurement mic and preamplifier; ACO Pacific 7200 PS9200 with 100$20 \mathrm{kHz}$ bandpass filter; Krohn-Hite 3700 ). Stimulus sound levels were converted to $\mathrm{dB}$ SPL peak equivalent by calibration against a continuous tone measurement (Bruel and Kjaer, 2203 sound level meter; ACO Pacific 7200 1/2-inch microphone).

\section{Sound level and threshold}

To simplify experimentation, sound levels used for latency and amplitude measurements corresponded with the $20,30,40,50$ and $60 \mathrm{~dB}$ settings of the step-attenuator, which were $87,78,69,59$ and $49 \mathrm{~dB}$ SPL, respectively. 
A reference BAEP was recorded for each test animal at $87 \mathrm{~dB}$ SPL. To determine threshold, sound pressure was reduced in $10 \mathrm{~dB}$ steps and at each step a 1024-sweep average was obtained. When the sound stimulus no longer produced any discernible peaks, the stimulus was increased $5 \mathrm{~dB}$, then decreased in $2 \mathrm{~dB}$ steps until the BAEP disappeared. Threshold was defined as the average of the lowest sound level at which a BAEP peak could be detected by visual inspection, and the highest sound level at which it was not visible.

Signal-to-noise ratio was determined by dividing the maximum peak amplitude of the BAEP for a given sound level by the average amplitude of the background noise.

\section{Drug effects}

The effects of $d$-tubocurarine chloride and Tricaine Methanosulfonate (TMS) on the BAEP were investigated with the following protocol: The BAEP was recorded for each frog immediately before drug administration. Dtubocurarine chloride was injected (i. m., $20 \mu \mathrm{g} / \mathrm{g}$ of bm), and a second BAEP recorded after 15 minutes. Alternatively, a frog was placed in a $3 \mathrm{mM}$ TMS bath and a BAEP recorded ( every 5 min.) until the frog no longer showed an eye blink reflex.

\section{Data transformation and statistical analysis}

When combining BAEP amplitude data from different frogs, the data were normalized to the percent of maximum amplitude. Individual animals had quite different BAEP maxima, and since I was interested only in the percent change in amplitude as a function of various stimulus parameters, each of the amplitude measurements for one animal (peak I and peak IV) 
were divided by the maximum respective peak I and IV amplitudes obtained for that animal under any stimulus condition. The maximum thus represented the best amplitude performance for that animal. The percent changes in amplitude were averaged across frogs, thereby reducing variability.

Linear best fit lines were added to the latency (absolute and interpeaks) versus sound level figures to help visualize trends.

Means were plotted with standard deviation (s.d.) or with $95 \%$ confidence intervals. In all cases the data obtained represent a single data set, thus standard deviation rather than standard error is the appropriate statisitcal measure. In most cases, the sample size (number of animals tested) was too small to justify the use of confidence intervals. When comparisons between data sets were made, however, confidence intervals were plotted. To better discern error bars on plots where means from different species overlap, the cap lengths for the respective error bars were made different in size, the widest was for $B$. terrestris and decreasing in size with $B$. americanus and $R$. pipiens in that order.

To test for significant differences between populations (data sets), the Mann-Whitney U-statistic was applied. This non-parametric equivalent to the Student's T-test is the most appropriate for my data.

\section{RESULTS}

\section{Waveform morphology}

Typical BAEPs for Rana pipiens, Bufo terrestris, and Bufo americanus are shown in figure 3 ( $A, B$, and $C$ respectively). The BAEPs are similar for each of the species, and all show at least four peaks. The major difference in 

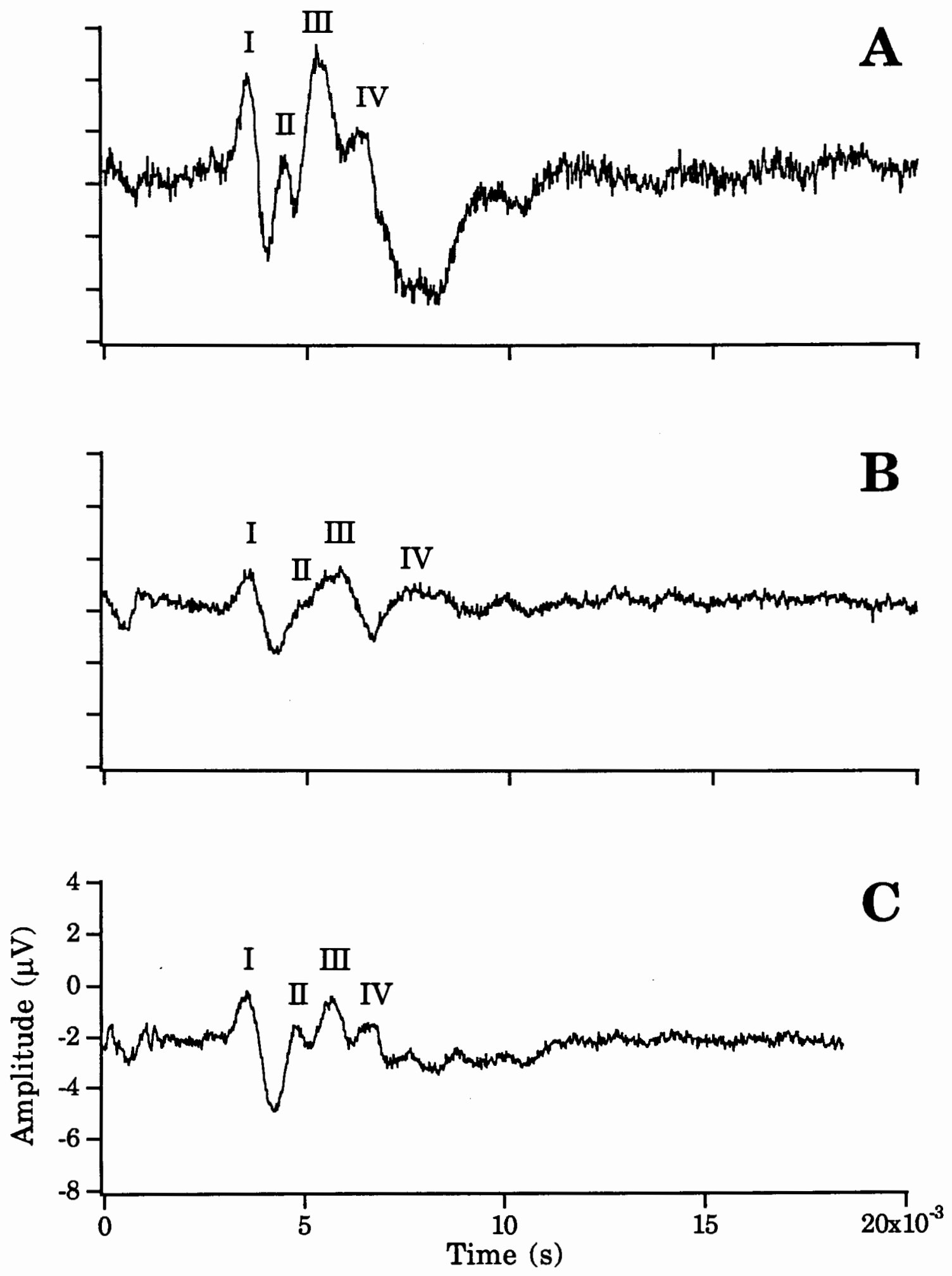

Figure 3. Typical BAEPs for three species of anurans. (A) Rana pipiens, (B) Bufo terrestris, (C) Bufo americanus. Major peaks of interest (I-IV) are labeled. 
the waveform morphology between the two genera is in peak II. For $R$. pipiens, peak II is separate from peak III, while for B. americanus and particularly for $B$. terrestris, peak II is less distinct and seems to blend into peak III. This difference is more readily apparent when the I-II interpeak latencies of the three species are compared.

\section{Sound level and Threshold}

A typical change in BAEP with stimulus sound level for $R$. pipiens is shown in figure 4. As the sound level of the acoustic stimulus was decreased, the overall latencies of the peaks increased and the amplitudes decreased.

The average signal-to-noise ratio was better for the $R$. pipiens than for the toads (Table I). As expected, the average signal-to-noise ratio declined as the sound level of the acoustic stimulus was decreased.

I determined a BAEP threshold as the average between the minimum sound level necessary to achieve a BAEP (Figure 4, $38 \mathrm{~dB}$ SPL) and the maximum sound level at which there was no BAEP (Figure 4, $35 \mathrm{~dB}$ SPL). The distribution of BAEP thresholds for $32 R$. pipiens , $26 R$. catesbeiana, 16 B. americanus and $11 B$. terrestris, is shown in figure 5 . The mean thresholds ( $\pm 95 \%$ confidence intervals) were: $36.1 \pm 1.44 \mathrm{~dB}$ SPL, $37.3 \pm 2.99 \mathrm{~dB}$ SPL, 45.6 $\pm 2.24 \mathrm{~dB}$ SPL and $50.6 \pm 4.57 \mathrm{~dB}$ SPL. The BAEP thresholds for the Ranid species were significantly lower than that for the Bufonids (MannWhitney $\mathrm{U}$ test, $\mathrm{p}<.01$ ). I tested the stability of BAEP threshold with repeated measures of individuals within a day and across multiple days (3-5). The mean and s.d. for the BAEP threshold of an individual within a day was $37.4 \pm 1.2 \mathrm{~dB}$ and across multiple days $38.1 \pm 1.6 \mathrm{~dB}$ (Table II).

Before a species comparison of latency data could be made, the latency data had to be temperature corrected. Rana pipiens data were normalized 

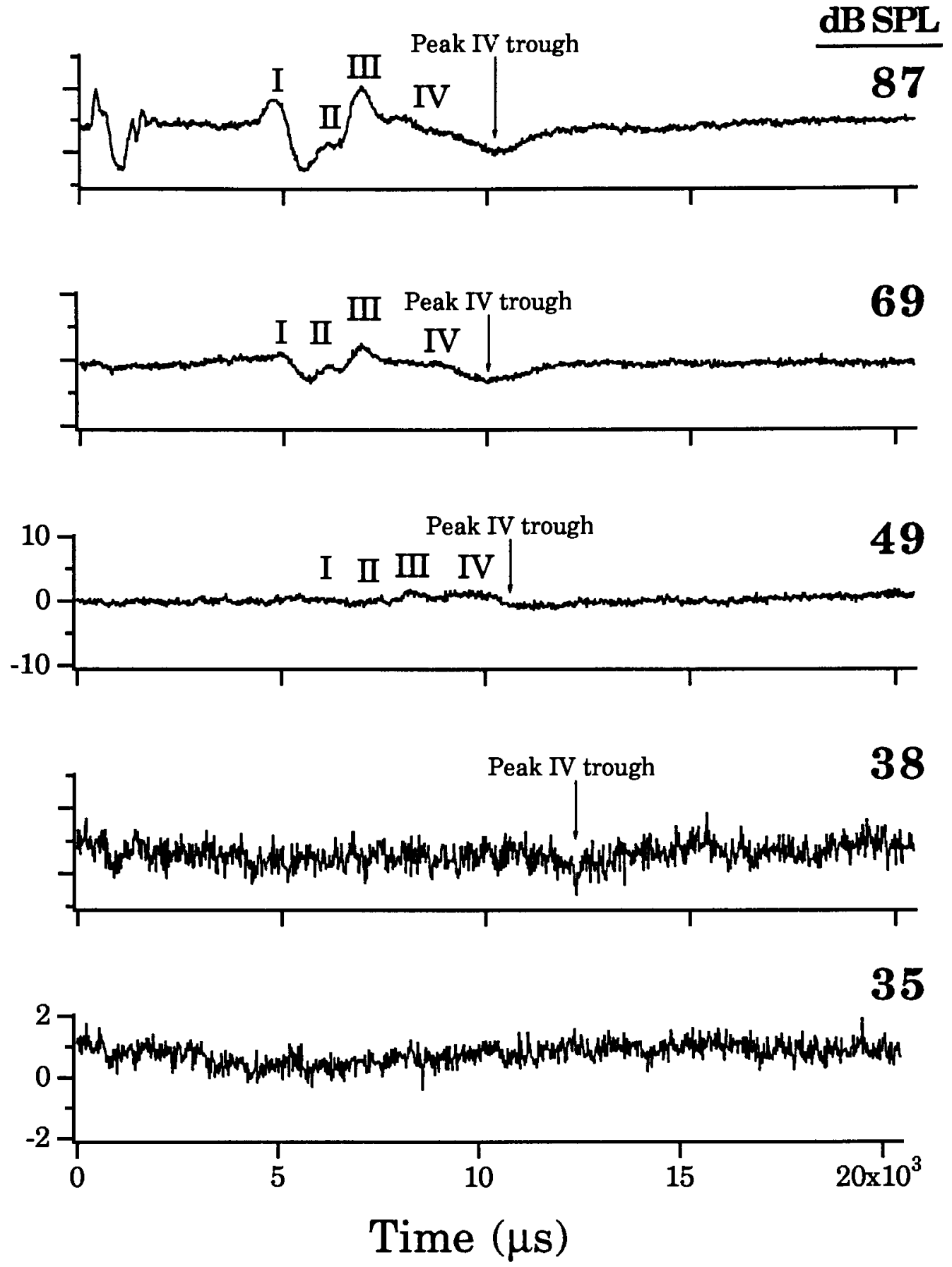

Figure 4. Typical BAEP change in response to stimulus level (dB SPL). In this series, threshold was between 38 and $35 \mathrm{~dB}$ SPL. (Threshold was determined as the average between the minimum sound level necessary to record ABR activity and the maximum sound level where $A B R$ activity was not present). Note the expanded y-axis scale for the 38 and 35 SPL traces. Each characteristic peak (positive) of the ABR is labeled (I, II, III, IV). The presence of the peak IV trough is a most sensitive indicator of BAEP activity. 
TABLE I

MEAN (N = 5, PER SPECIE) SIGNAL-TO-NOISE RATIOS WITH STANDARD DEVIATIONS FOR THREE SPECIES OF ANURANS AT DIFFERENT TEST STIMULUS LEVELS (dB SPL)

Rana pipiens

Signal to noise ratio:

Signal to noise ratio:

Signal to noise ratio:

\author{
$7.4 \pm 2.19$
}

87

78

$\underline{69}$

$\underline{59}$

$\underline{49}$
$4.4 \pm 1.59 \quad 3.6 \pm 1.59$
$4.0 \pm 2.10$
$5.0 \pm 0.40$
Bufo americanus

87

78

69

$\underline{59}$
Bufo terrestris

$\begin{array}{llll}87 & 78 & 69 & 59\end{array}$ 

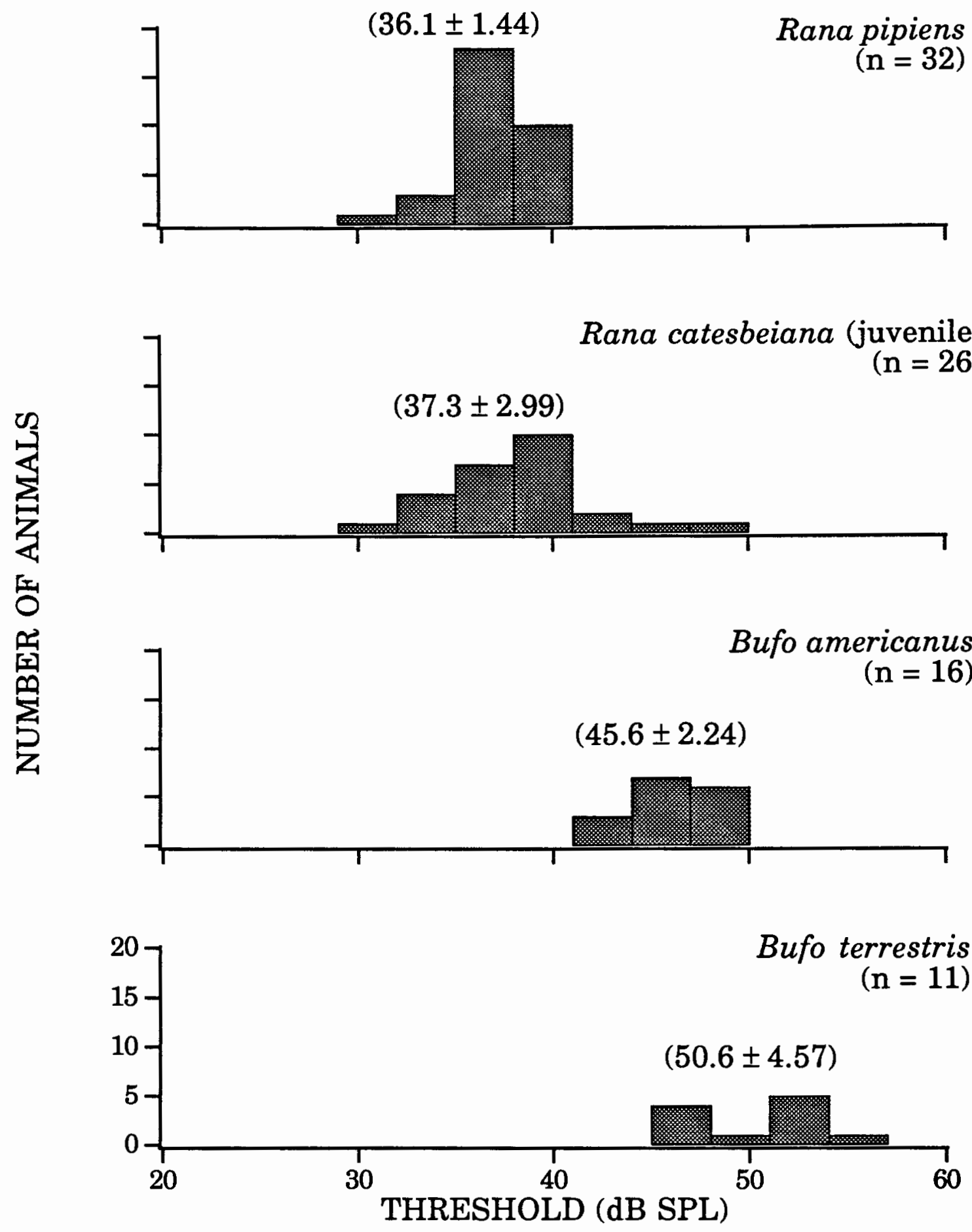

Figure 5. Distributions of BAEP thresholds for two species of Rana and $B u f o$. Bufonid BAEP thresholds were significantly higher than BAEP thresholds for Ranids (Mann-Whitney U test, $\mathrm{p}<.001$ ). Mean BAEP thresholds and 95\% confidence intervals are given above each distribution. Juvenile $R$. catesbeiana had a wider distribution of thresholds compared to adults of other species. 
TABLE II

AVERAGES AND STANDARD DEVIATION (S.D.) FOR SELECTED PARAMETERS OF THE BRAINSTEM AUDITORY EVOKED POTENTIAL IN RANA PIPIENS (N=4)

Error Within a Day

Average

37.4

\begin{tabular}{|c|c|}
\hline dB SPL & Average \\
\hline 87 & 2.55 \\
\hline 78 & 2.77 \\
\hline 69 & 3.00 \\
\hline 59 & 3.06 \\
\hline 49 & 3.51 \\
\hline
\end{tabular}

\begin{tabular}{cc} 
dB SPL & Average \\
\hline 87 & 1.21 \\
78 & 1.24 \\
69 & 1.27 \\
59 & 1.34 \\
49 & 1.38
\end{tabular}

dB SPL

87

78

69

59

49

dB SPL

87

78

69

59

49

dB SPL Average

87

78

69

59

49

$\frac{\mathrm{dB} \text { SPL }}{87} \quad \frac{\text { Average }}{81.7}$

78

69

59

49
0.89

0.96

1.08

1.14

1.53

Average

1.80

1.80

1.80

1.69

2.01

95.8

82.6

56.8

42.2

32.4

81.7

89.8

78.0

59.3

52.6 s.d.

1.15

$\frac{\text { s.d. }}{0.04}$

0.05

0.08

0.04

0.08

I-II Interpeak Latency (ms)

s.d.

0.06

0.05

0.22

0.14

0.14

dB SPL

Error Across Days

II-III Interpeak Latency (ms)

\begin{tabular}{lcccc}
\hline s.d & dB SPL & Average & s.d \\
0.04 & 87 & 0.94 & 0.09 \\
0.06 & 78 & 0.96 & 0.07 \\
0.09 & 69 & 1.05 & 0.12 \\
0.11 & 59 & 1.11 & 0.10 \\
0.34 & 49 & 1.21 & 0.09
\end{tabular}

III-IV Interpeak Latency (ms) s.d.

0.19

0.14

dB SPL

0.10

0.23

87

49

0.16

Peak I Amplitude (\% of max.)

s.d.

6.0

17.6

12.3

16.6

dB SPL

8.9

49

Peak IV Amplitude (\% of max.)

s.d.

11.6

dB SPL

87

78

10.8

15.5

17.8

\begin{tabular}{cc} 
Average & s.d. \\
\hline 1.87 & 0.24 \\
1.81 & 0.18 \\
1.86 & 0.14 \\
1.82 & 0.21 \\
2.08 & 0.22
\end{tabular}

Average

98.0

77.0

51.7

37.4

33.7

Average

82.0

92.5

82.4

58.7

53.1

s.d.

0.24

0.18

0.14

0.22

s.d.

3.4

13.5

10.4

6.7

11.9 s.d.

10.6

8.4

14.3

12.8

20.3
87

78

69

59

49

78

69

59

87

78

69

59

69

s.d.

0.11

0.04

0.15

0.34

0.04

s.d.

0.10

0.08

0.12

0.10

0.16

s.d.

0.09

0.12

0.10

0.09

59

49 
according to the mean recording temperature for the bufonid species; the frog data were recorded at an average temperature of $21^{\circ} \mathrm{C}$ and the toad data were recorded at an average temperature of $23^{\circ} \mathrm{C}$. Based on our temperature experiments (below), this difference in temperature may have had a significant effect on the comparison of latencies between species. To perform the normalization, I subtracted (if the temperature of the frog was below $23^{\circ} \mathrm{C}$ ) or added (if the temperature of the frog was above $23^{\circ} \mathrm{C}$ ) the latency increment which corresponded to the difference between the two temperatures for the Ranids.

The average absolute latencies (peaks I, II, III, IV) in response to five different sound levels for $R$. pipiens, four different sound levels for $B$. americanus and four different sound levels for B. terrestris are shown in figures 6. A similar plot of interpeak latency is given in figure 7. I plotted best linear fit lines and 95\% confidence intervals for each specie for all of the latency versus sound level plots. As the sound level of the stimulus was decreased ( $10 \mathrm{~dB}$ increments), all of the absolute peak latencies increased linearly (Figure 6A-C), while interpeak latencies remained constant (Figure 7A-C). All three species responded in a similar fashion when the sound level of the stimulus was decreased. Preliminary data for $R$. pipiens (not shown) may suggest that below $49 \mathrm{~dB}$ SPL absolute peak latencies increase in a nonlinear (exponentially) fashion as the sound level is decreased.

Interpeak latencies remained constant, within experimental error over the sound levels used for each of the species (Figure 7A-C). Between genera, there were significant differences at 69,78 and $87 \mathrm{~dB}$ SPL for I-II interpeak latencies (Mann-Whitney U-test, p<.01). Rana pipiens had a shorter I-II interpeak latency than the bufonid species. This difference between the 

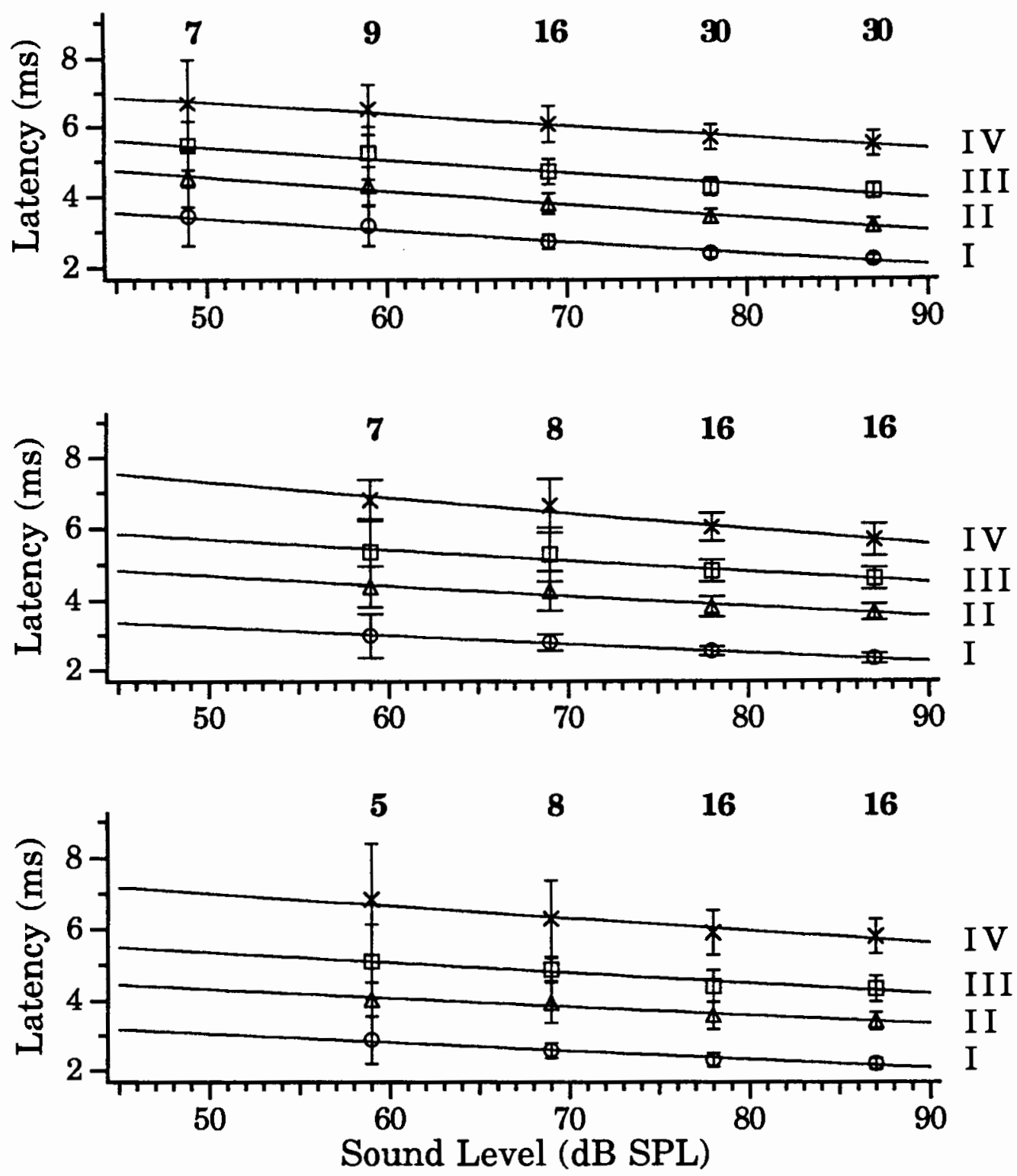

Figure 6. Absolute peak latencies versus sound level for four BAEP peaks in three species of anurans. Unfilled circles = peak I; unfilled triangles = peak II; Unfilled squares = peak III; "X" = peak IV.

Points are means with $95 \%$ confidence intervals. Number of animals tested are indicated above each test sound level (in bold) for all species. Linear fits for each curve are:

(A) Rana pipiens, peak I: $\mathrm{y}=5.03-0.033 \mathrm{x}$; peak II: $\mathrm{y}=6.44-0.038 \mathrm{x}$; peak III: $\mathrm{y}=7.22-0.036 \mathrm{x}$; peak IV: $\mathrm{y}=8.40-0.034 \mathrm{x}$.

(B) Bufo americanus, peak I: $\mathrm{y}=4.5-0.025 \mathrm{x}$; peak II: $\mathrm{y}=6.12-0.029 \mathrm{x}$; peak III: $\mathrm{y}=7.19-0.030 \mathrm{x}$; peak IV: $\mathrm{y}=9.49-0.044 \mathrm{x}$.

(C) Bufo terrestris, peak I: $\mathrm{y}=4.24-0.024 \mathrm{x}$; peak II: $\mathrm{y}=5.53-0.024 \mathrm{x}$; peak III: $\mathrm{y}=6.78-0.029 \mathrm{x}$; peak IV: $\mathrm{y}=8.72-0.034 \mathrm{x}$. 

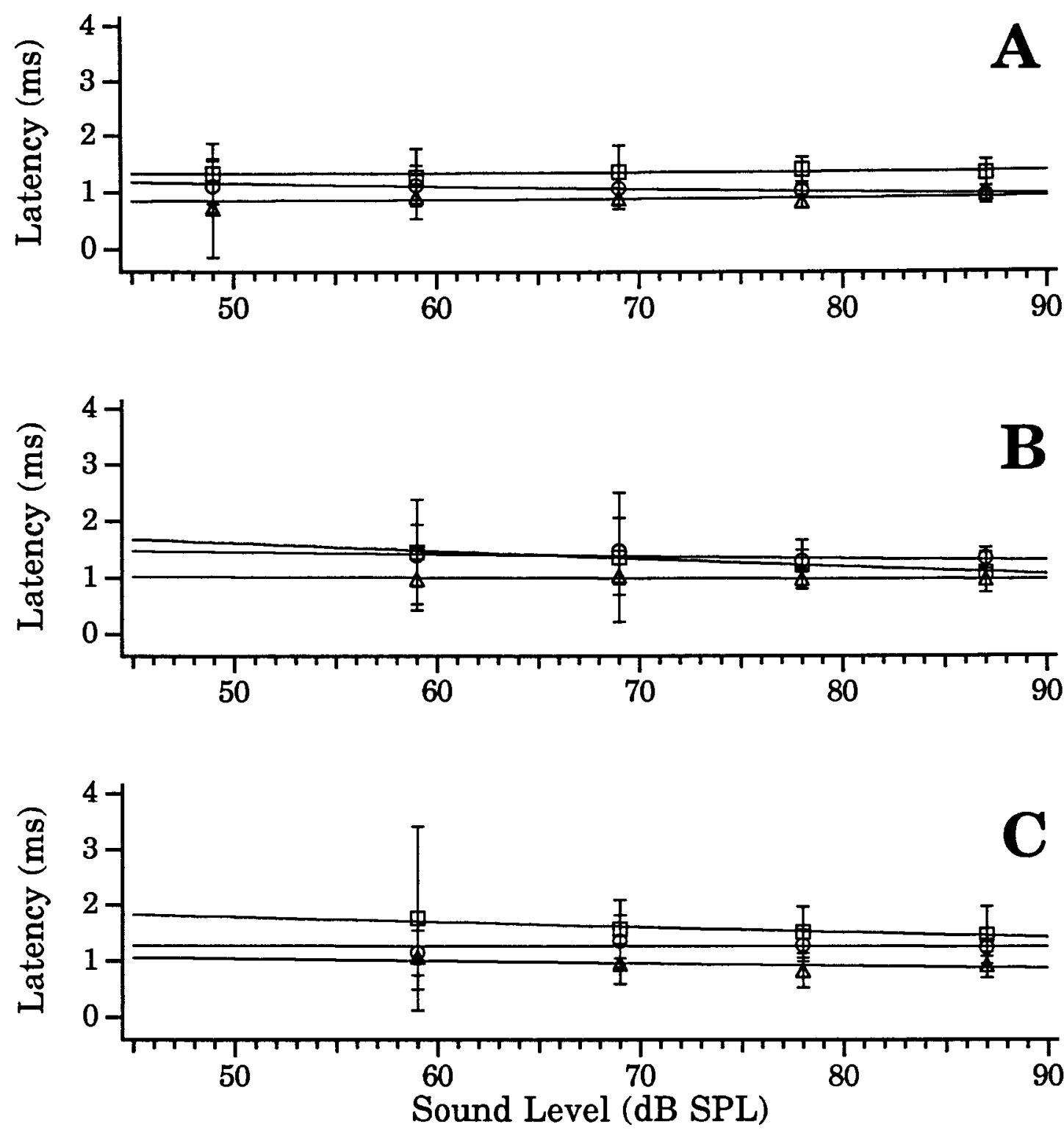

Figure 7. Interpeak latencies versus sound level for three BAEP interpeaks in three species of anurans. Unfilled circles = I-II interpeak; Unfilled triangles = II-III interpeak; Unfilled squares = III-IV interpeak. Points are means with 95\% confidence intervals. Number of animals for each sound level is the same as in figure 6 . The linear fits for each response are:

(A) Rana pipiens, I-II interpeak: $\mathrm{y}=1.39-0.005 \mathrm{x}$; II-III interpeak: $\mathrm{y}=0.77+0.001 \mathrm{x}$; III-IV interpeak: $\mathrm{y}=1.27+0.001 \mathrm{x}$.

(B) Bufo americanus, I-II interpeak: $\mathrm{y}=1.64-0.004 \mathrm{x}$; II-III interpeak: $\mathrm{y}=1.07-0.001 \mathrm{x}$; III-IV interpeak: $\mathrm{y}=2.29-0.014 \mathrm{x}$.

(C) Bufo terrestris, I-II interpeak: $\mathrm{y}=1.28+0.000 \mathrm{x}$; II-III interpeak: $y=1.25+0.004 x$; III-IV interpeak: $y=2.24-0.009 x$. 
species was mentioned earlier when the comparison of the waveform morphologies was made (Figure 3). At the lower sound levels, the I-II interpeak latencies for all species were not significantly different.

To analyze the change in amplitude of BAEP peaks in response to sound level change I used peaks I and IV, since they were the most prominent and stable of the four peaks. These peaks were also selected based on their points of origin in the nervous system. Peak I has been shown to originate from the VIIIth nerve (Seaman, 1991) and therefore represents the peripheral portion of the nervous response. Based on the latency of peak IV compared with latencies reported for auditory active nuclei (Feng, 1991; Fuzessery and Feng, 1983), I estimate this peak to originate in the brain stem, possibly in the superior olivary nucleus and therefore represents a CNS evoked response. The effects of sound level change on the amplitudes of peak I and peak IV of the BAEP for $R$. pipiens, B. americanus and B. terrestris are shown in figure 8.

For all species tested, the amplitude of peak I decreased with decreasing sound level (Figure $8 \mathrm{~A}$ and $\mathrm{C}$ ). Peak I and IV amplitudes were normalized to percent of maximum amplitude. Peak I amplitude for each of the anurans changed at similar proportional rates with the sound level of the acoustic stimulus.

While peak I amplitude did not saturate at the highest test sound levels used, there is some indication that peak IV amplitude did saturate above $75 \mathrm{~dB}$ SPL (Figure $8 \mathrm{~B}, \mathrm{D}$ ). Peak IV amplitude for the ranid was greater than the bufonids for all test sound levels. 

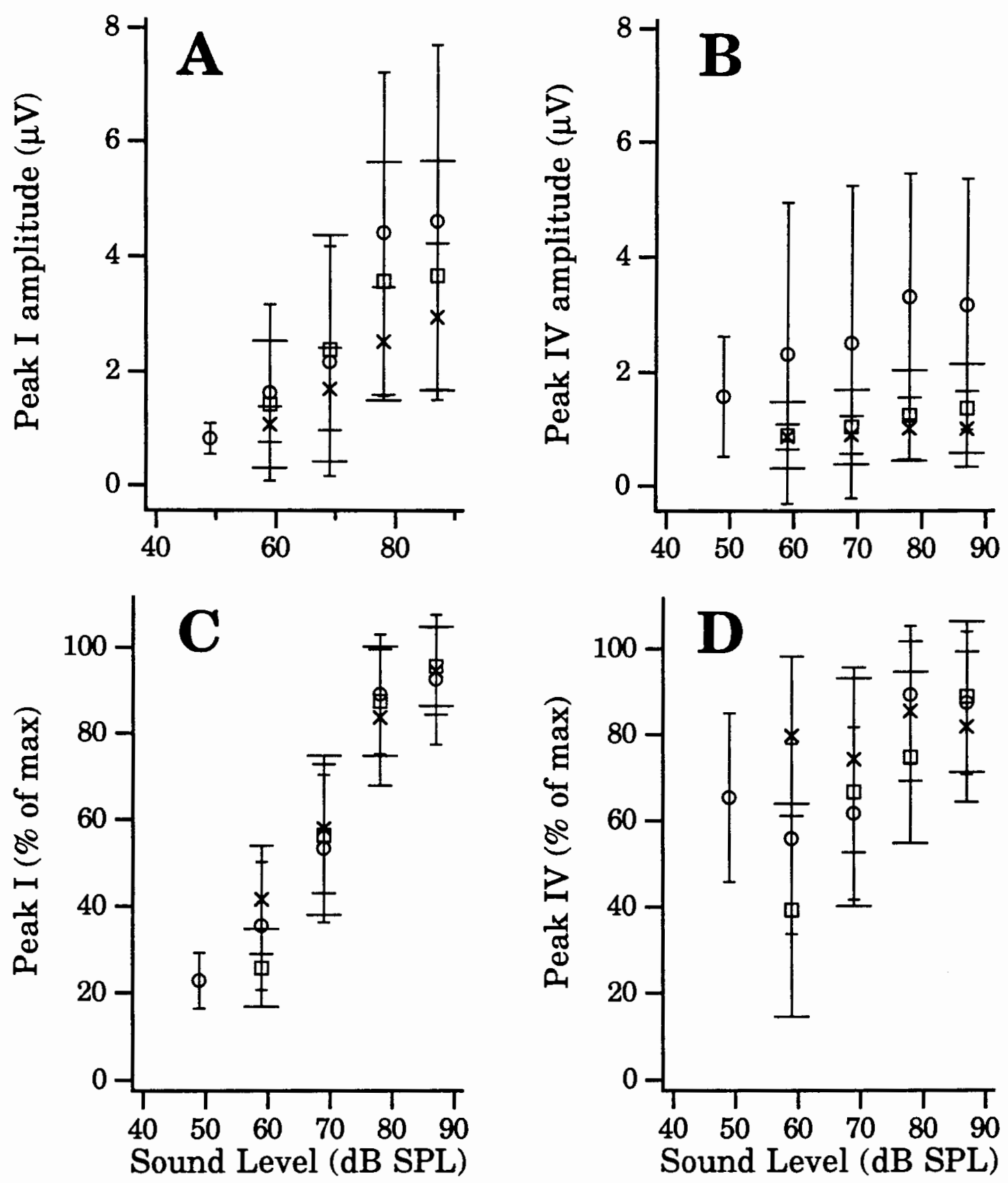

Figure 8. A comparison of the peak I and IV amplitudes for $R$. pipiens, $B$. Americanus and $B$. terrestris versus sound level.

Points are means with standard deviations. Number of animals for each test sound level is the same as in figure 6. For all panels (A-D), Rana pipiens: Unfilled circles; Bufo terrestris: Unfilled squares; Bufo americanus: X's.(A) The peak I amplitudes for all species tested. (B) The peak IV amplitudes for all species tested. (C) The percent of maximum amplitude for peak I amplitudes for all species tested. (D) The percent of maximum amplitude for peak IV amplitudes for all species tested. 


\section{Drug effects}

BAEPs were recorded both before and after frogs were injected with dtubocurarine chloride. D-tubocurarine chloride reversibly blocks synaptic transmission in the efferent nervous system by occupying postsynaptic receptors for the neurotransmitter acetylcholine (Kuffler, et al., 1984). There was no significant difference in peak I and the three interpeak latencies between the two groups ( $p<.05)$. There was a slight but significant decrease in BAEP threshold of $1.66 \pm 0.78$ (s.d.) $\mathrm{dB}$ ( $\mathrm{p}<.01$ ), however.

The anesthetic TMS had an impact on the overall waveform morphology of the BAEP (Figure 9). As the time of exposure to the TMS was progressively increased, all four peaks decreased in amplitude and increased in latency. Figure 9B shows the BAEP at the point where all the peaks begin to become affected. At this exposure time, peaks III and IV are more reduced than peaks I and II. With further exposure (Figure 9C) the CNS peaks III and IV nearly had disappeared while peak I remained prominent. At this final exposure level the frog was unconscious (no eye blink reflex). This response of the BAEP to the dose of the TMS suggests that the TMS affects the central nervous system before the peripheral nervous system.

\section{DISCUSSION}

\section{Waveform morphology}

A typical anuran BAEP has four peaks. The neural generators responsible for the production of the peaks have not been found, but rather predicted based on comparing the absolute latencies of the peaks with the latencies of various neural generators already shown in previous studies. As in the mammalian BAEP, peaks I and II, which range from $2.0-5.0 \mathrm{~ms}$ and 

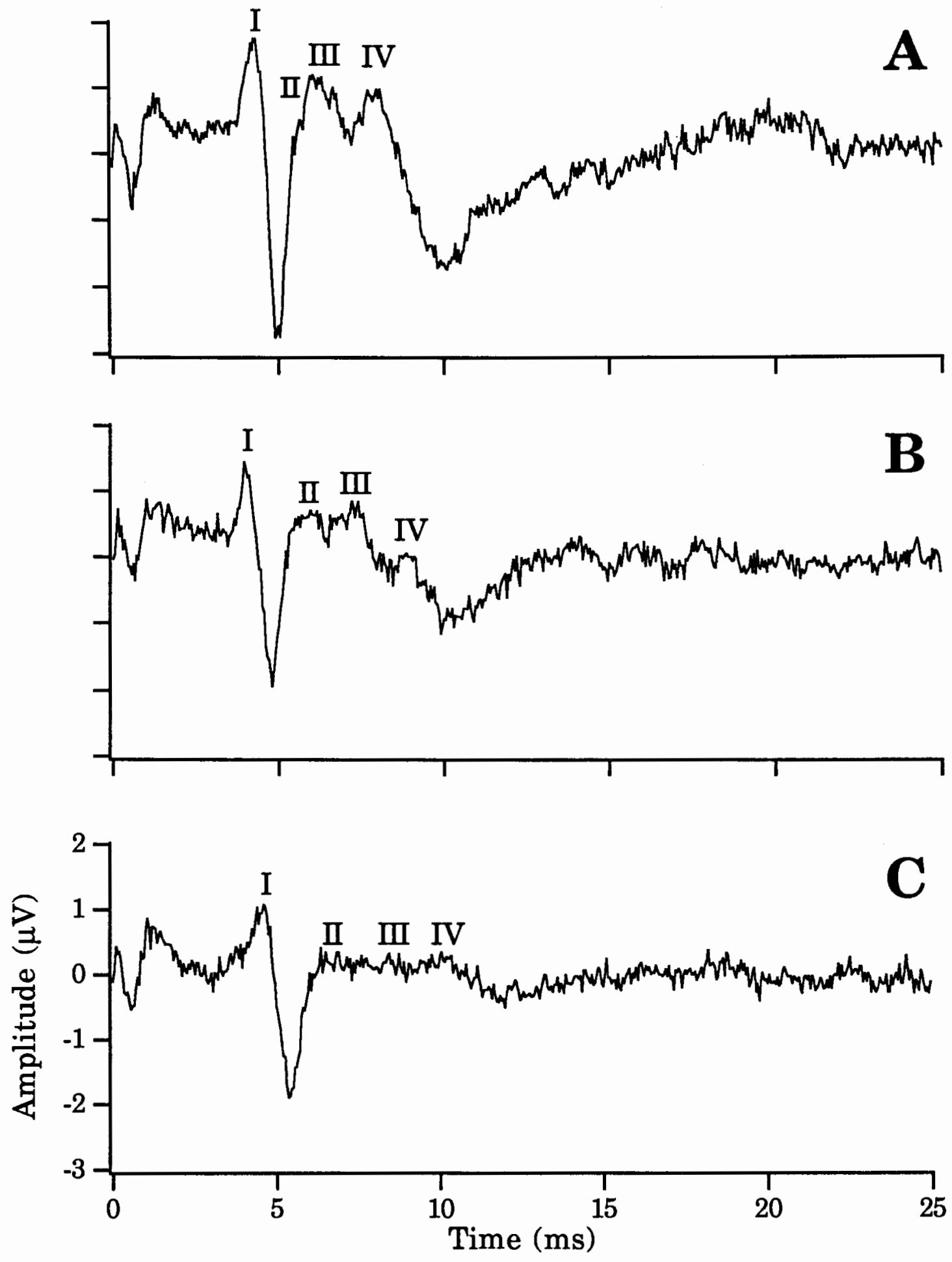

Figure 9. Effects of TMS anesthetic on the BAEP. (A) 0 minutes exposure; (B) 5 minutes exposure; (C) 10 minutes exposure. The more central (nervous system) peaks, III and IV, are affected before the peripheral peaks, I and II. 
3.2 - $6.5 \mathrm{~ms}$ in latency, respectively, seem to arise from different portions of the VIIIth nerve (Seaman, 1991). Peak III, which ranges from 4.2 - $7.5 \mathrm{~ms}$ in latency, is comparable to latencies reported for the DMN (Fuzessery and Feng, 1983). Peak IV, 5.7-9.0 ms, is probably SON and not TS, since the latencies reported for TS activity, using acute tungsten electrode multi-unit recordings, are greater than $10 \mathrm{~ms}$ (Hubl and Schneider, 1979a).

Ranid BAEPs differ from bufonid BAEPs with respect to peak II latency. When comparing waveform morphologies, peak II for bufonids was not isolated, but rather had merged with the more prominent peak III. A comparison of the I-II interpeak latencies between the genera shows that peak II is further from peak I (increased latency) for the bufonids than for the ranids.

There are two hypotheses which may explain the I-II interpeak difference between the genera. The first would assume that the neural circuitry is the same for both genera and therefore the neural generator for peak II is different for Rana and Bufo. The second hypothesis would require that the neural generators for peak II are the same for both genera, but instead there is a change in the pathway between peaks I and II causing a delay in the signal. These hypothesis require that peaks I and III have the same origin and features in both genera. this is likely since peaks I and III are very prominent in the BAEP, suggesting they arise from specific centers which are highly synchronous in nature. Since the II-III interpeak latency is the same for both genera, I favor the hypothesis of increased delay.

\section{Sound level and Threshold}

BAEP thresholds for the ranids were significantly lower than the BAEP thresholds in bufonids. The mean difference between genera was 
approximately $10 \mathrm{~dB}$ and while this is a substantial amount, it is less than the $30 \mathrm{~dB}$ difference reported by Wever (1985) using an inner ear gross potential technique. This difference is most likely a function of the different recording techniques used by Wever and those used in the present study. Unfortunately, the reference and criterion used to describe threshold in the Wever study are difficult to interpret and do not permit reconciling the discrepancy.

A small part of the BAEP threshold difference between the ranids and bufonids may be explained by a smaller signal-to-noise ratio for the bufonids, resulting in an apparent increase in the BAEP threshold. While the noise levels for the ranids and the bufonids were similar in amplitude, the amplitudes of the bufonid peaks were markedly lower than the amplitudes found in the ranids. In particular, Peak IV amplitude for the bufonids was $\sim 40 \%$ of that found for the ranids. Since BAEP threshold is based on the presence of the various peaks, the smaller amplitude of the bufonid peaks may have resulted in higher thresholds.

There are at least two possible explanations for the smaller amplitudes associated with the bufonids. The first is that the effect reveals a true performance difference between the bufonid and ranid auditory systems, manifested as a lack of sensitivity. There is anatomical evidence for a true performance difference. The area of the tympanum for a $B$. americanus is $\approx 20 \mathrm{~mm}^{2}$ (Wever, 1985), while the area of the tympanum is much larger $(6.25 \mathrm{x})$ in a ranid of comparable size. I mentioned before that the effective sound pressure transmitted to the inner ear from the tympanum is a function of the ratio of the tympanum to the oval window areas. Although there are no reported measurements of oval window size for these animals, if the ranid 
and bufonid oval windows are similar in size, the relatively larger ranid tympanum will yield more efficient sound coupling to the inner ear $(\approx 16 \mathrm{~dB}$ more sensitive). A second explanation for larger BAEP peaks in ranids involves differences in skin surface the external surface of ranids is wetter than bufonids which may have allowed for better contact with the common of the differential amplifier, thus producing better common mode rejection and a superior apparent signal-to-noise ratio. The common electrical connection was made through the copper screen cage in which the animal sat during testing. A wetter ventral skin surface could have provided a lower resistance (impedance) path. I did not make impedance measurements for these animals to check analyze this affect.

In comparing the BAEP thresholds in the present study with others found in the literature for $R$. catesbeiana, BAEP threshold was 5 - $20 \mathrm{~dB}$ less sensitive than behavioral threshold (Megela-Simmons, et al., 1985) and approximately the same as that found by other neurophysiological techniques using tone bursts (Lombard, et al., 1982).

Since there is a narrow and predictable difference between BAEP thresholds and thresholds obtained using other methods, the relative ease of acquiring BAEPs make it a better method with which to determine thresholds for anurans, provided frequency-specific measures are not required. Although behavioral thresholds can be repeated in the same animal over an extended period of time, it takes a lot of time and effort to train the animals to behaviorally respond to a stimulus and this training is not guaranteed to hold once the animal has been placed under a physiological stress. The limitations of using more invasive neural recordings are (1) a shorter recording period is possible with acute preparations, (2) the more 
intensive surgery required adds trauma and lower yields of good preparations, (3) typically a small subset of auditory system function may be sampled and (4) the time required to process a larger number of animals is much greater.

The $R$. catesbeiana used for BAEP threshold determination were in varying stages of juvenile development, while adults were used from the other species. The different stage of development could account for the greater range of thresholds found for the $R$. catesbeiana relative to the other species, since the auditory system is still developing during the first two years post-metamorphosis (Capranica, 1976). The BAEP would thus be a useful technique for assessing developmental changes in auditory system performance.

There is a degree of error associated with subjective determination of BAEP threshold. Presence of the BAEP waveform at the lower sound levels is difficult to determine with the attendance of background noise. Only with considerable practice at interpreting BAEP recordings at varying sound levels, may a researcher develop the intuition necessary to precisely determine BAEP threshold. The BAEP threshold determination error in this study was $\pm 1.2 \mathrm{~dB}$ (at $37.4 \mathrm{~dB}$ SPL) within a day and $\pm 1.6 \mathrm{~dB}$ (at $38.1 \mathrm{~dB}$ SPL) across multiple days (3-5). This is quite good and within the $3 \mathrm{~dB}$ limit accepted for other neurophysiological determinations of threshold. Further refinement could be obtained by cross-checking threshold with another researcher equally trained in the technique.

Latency changes with to decreases in acoustic stimulus sound level were the same for all species tested. All absolute peak latencies increased linearly with decreasing stimulus sound level, while the three interpeak 
latencies remained relatively unchanged across all test sound levels.

Preliminary data suggests that at lower sound levels ( $<49 \mathrm{~dB})$ absolute peak latencies may increase nonlinearly (not shown).

Over a wide range of test sound levels, absolute peak latencies increased linearly while interpeak latencies for the most part remained stable. This suggests that stimulus sound level had little effect on signal conduction within the nervous system. The peak II, III, IV absolute latencies did change with sound level, but this is certainly a reflection of the peak I effect. Why should a decreased sound level increase latency to peak I, but not increase the interpeak latencies? Indeed, why is there a latency change at all due to test level? A delay is also apparent in mammalian BAEP responses to decreases in sound level of the stimulus. In mammals, the primary reason for the latency shift due to sound level is a change in the spectral quality of the click as its level changes (Don and Eggermont, 1978). As a click sound level decreases, the frequency which is most dominant in the click decreases. Based on the tonotopic organization of the basilar membrane, this shift in the spectrum of the click results in the traveling wave propagating further along the basilar membrane before peak excitation of the sensory epithelium occurs. The lengthening of the traveling wave propagation time thus results in an evoked potential peak occurring at a longer delay. A traveling wave phenomenon is also likely in the amphibian papilla of frogs, since the phasefrequency relationship in the frog papilla is similar to the relationship observed in the mammalian cochlea (Hillery and Narins, 1984). Therefore I presume the latency change in frogs is of the same nature as mammals and due to level-dependent spectral changes in the test stimulus. Such an effect 
depends on a traveling wave and is thus restricted to the periphery. It is not a general nervous system effect.

Another way to consider the present data is that the presence of latency shifts support the notion of a traveling wave in anurans. With this in mind, it would be useful to produce spectrally-filtered BAEP stimuli which selectively stimulate only the basilar papilla. There is no evidence for a traveling wave in the basilar papilla thus no level-latency dependence should be observed in a BAEP of exclusively basilar papilla origin.

The amplitudes of peaks I and IV vary between individuals and across species to a much greater extent than equivalent latency data. When the data are normalized, using the percent of maximum amplitude, I found that the amplitudes of both peaks responded in similar fashion to stimulus sound level changes for all three species tested.

As the sound level of the acoustic stimulus is increased, the amplitude of peak IV saturates (reaches maximum) before peak I amplitude. This would mean that at sound pressures about $75 \mathrm{~dB}$ SPL, changes in peak I amplitude have no effect on peak IV amplitude. If amplitude is a reflection of the amount of neural activity occurring, then central auditory activity is at its maximum above $75 \mathrm{~dB}$ SPL and at sound levels lower than $75 \mathrm{~dB}$ SPL not all auditory neurons are active.

\section{Drug effects}

D-tubocurarine chloride (DTC) had no significant effect on the anuran BAEP. This would allow for interesting future experiments. There have been many questions raised as to the function of the efferent innervation of the inner ear. It is thought that the efferent innervation may be involved in protection of the auditory apparatus from overstimulation and the anuran 
auditory system is potentially an excellent model to address this question. The amphibian papilla has efferent innervation while the basilar papilla does not (Ronken, 1990; Wilczynski and Capranica, 1984). One can selectively record BAEPs which are received from the amphibian or basilar papilla afferent innervation, using acoustic clicks spectrally filtered such that they can only excite either endorgan. One could then expose two groups of frogs, a control and an experimental group which is injected with the DTC, to high levels of noise. The DTC will reversibly block the efferent nerves and if there is a difference in the threshold shifts of the amphibian papilla associated BAEP between the control and DTC-injected frogs, we can attribute that difference to the efferent innervation.

The anesthetic TMS had a profound effect on the entire BAEP, but especially on the more central peaks, confirming its efficacy as an anesthetic. If the anesthetic is used during BAEP implantation or at any other time, the animal should be allowed to recover over an extended period of time ( $>6 \mathrm{hrs}$.), so that there will be no lingering effects of the TMS on the BAEP. 


\section{CHAPTER II}

\section{EFFECTS OF TEMPERATURE ON THE ANURAN BRAINSTEM AUDITORY EVOKED POTENTIAL}

\section{INTRODUCTION}

There are a number environmental factors that may have an effect on the calling behavior and hearing of anurans (Zweifel, 1968). Of these factors, temperature is the one most often studied (Hubl and Schneider, 1979b; Mohneke and Schneider, 1979; Rose, et al., 1985). Being an ectotherm, the anuran is unable to metabolically regulate its body temperature. Since the temperature of the surroundings may vary greatly, the body temperature of an anuran may vary as well.

There are several reports which indicate temperature coupling of mating call dominant frequency preference and dominant frequency produced. For example, in $H$. versicolor, females prefer calls with temporal properties similar to those produced by a conspecific male at about the same body temperature as their own (Gerhardt, 1978). For $H$. versicolor and $H$. chrysoscelis, temperature coupling of call frequency and hearing selectivity is temporally processed in the auditory midbrain (torus semicircularis) (Rose, et al., 1985).

Temperature effects on the threshold of hearing in many anurans have also been studied (Hubl, et al., 1977; Hubl and Schneider, 1979b; Mohneke and Schneider, 1979). In these studies, multi-unit activity at the level of the torus semicircularis (TS) was measured at five selected temperatures. 
Threshold was defined as the sound pressure at which neuronal responses were barely recordable. They found that as the temperature of the animal is decreased the sound pressure necessary to achieve threshold of activity, as defined, increases. The threshold of activity for the TS increases in a nonlinear fashion as the body temperature of the animal decreases. The latency of response to the acoustic stimuli for the TS was also measured and was found to increase in a nonlinear fashion as the temperature of the animal decreases.

In the current study, I used the BAEP to monitor changes in threshold and in the latencies of the four BAEP peaks in response to changes in temperature. I used two sets of frogs (Rana pipiens) acclimated to different temperatures to determine if temperature acclimation has an influence on BAEP threshold. An attempt is made to correlate the nonlinear shift in BAEP threshold in response to a decrease in temperature with similar shifts in the other parameters of the BAEP.

\section{METHODS}

Frogs were acclimated at either $21^{\circ} \mathrm{C}$ or $14^{\circ} \mathrm{C}$ for at least three weeks. Following acclimation, each animal was implanted with an BAEP recording electrode pair (see above) and additionally with a type $\mathrm{T}$ thermocouple (copper-constantin) held in place using the same procedure as that used for the BAEP electrode wires. After the surgical procedure (ca. 30 minutes), the animal was transferred back to the appropriate acclimation chamber for a minimum of 48 hours. Immediately before recording, $7 \mathrm{~m}$ of copperconstantin lead wire was soldered to the short thermocouple previously affixed to the frog. The lead wires were routed out of the sound isolated 
chamber thus permitting remote brain temperature monitoring during testing (Omega HH-25TC digital thermometer). Test frogs were transiently warmed and cooled by placing them on a copper manifold through which water at the required temperature was pumped (Forma Scientific 2095 water bath). Each frog was cooled from $21^{\circ} \mathrm{C}$ to an individual-dependent minimum, then heated to an individual-dependent maximum, and finally cooled to the starting (ambient) temperature. The BAEP was recorded at $87 \mathrm{~dB}$ SPL, 78 $\mathrm{dB}$ SPL, and threshold SPL at approximately $1^{\circ} \mathrm{C}$ intervals.

\section{Calculations}

BAEP threshold shift was calculated as the difference between ambient temperature $\left(21^{\circ} \mathrm{C}\right)$ BAEP threshold $(=33.7 \mathrm{~dB}$ SPL) and BAEP threshold at other temperatures. The divergences between the interpeak latencies were calculated by subtracting the II-III interpeak latency from IIIIV and I-II interpeak latencies obtained at similar temperatures.

\section{RESULTS}

\section{Temperature effects on the BAEP}

Nine Rana pipiens were acclimated to $21^{\circ} \mathrm{C}$ for at least three weeks. During an experimental test, the acclimated frogs were transiently warmed from $6^{\circ} \mathrm{C}$ to $26^{\circ} \mathrm{C}$ at a rate of $0.2^{\circ} \mathrm{C} /$ per minute. Figure 10 displays representative BAEPs for $R$. pipiens at six different temperatures. While $R$. pipiens was warmed, the duration of the BAEP condensed as the latencies to the peaks and the breadths of the individual peaks of the BAEP decreased in time. 

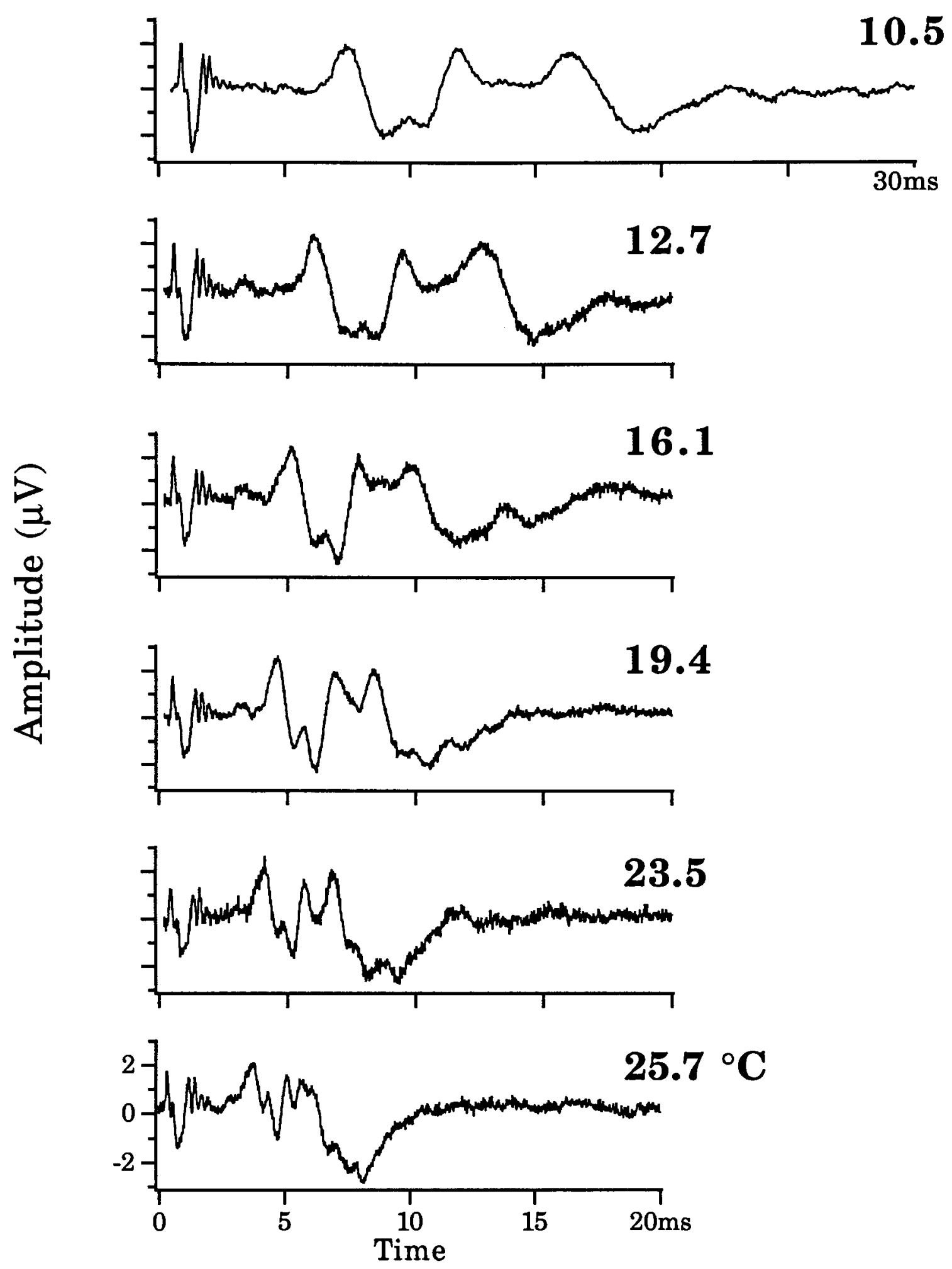

Figure 10. BAEP change with temperature. The BAEP of one frog shown at six selected temperatures (at right of each panel). The BAEP recorded at $10.5{ }^{\circ} \mathrm{C}$ (Top BAEP) has an extended time axis. 
As the frogs were transiently warmed, BAEPs were recorded at $87 \mathrm{~dB}$ SPL and threshold determined approximately every $1.0^{\circ} \mathrm{C}$. Figure 11 shows the effects of temperature on the BAEP threshold for frogs acclimated to $21^{\circ} \mathrm{C}$. For most frogs, BAEP threshold was stable between 20 and $26^{\circ} \mathrm{C}$ (Figure 11, B-E are examples). Below this temperature range, the BAEP threshold increased markedly as the temperature was decreased. The "transition temperature" at which a frog changed from a more stable to a temperature-dependent BAEP threshold was different for each frog. Transition temperature was estimated by applying linear best fit lines to the relatively more stable region and the increasing region of the thresholdtemperature curve. The temperature at which the lines intersected was the transition temperature. The transition temperature ranged from 16 to $23^{\circ} \mathrm{C}$ with the average transition temperature of $19.9 \pm 0.8$ (s.d.) ${ }^{\circ} \mathrm{C}$.

The peak I latency and the interpeak latencies differ in their response to the increase in temperature (Figure 12). Peak I latency changed exponentially with temperature. And unlike what was seen in the BAEP threshold response to temperature, peak I latency did not remain stable across the temperature range $\left(20-26^{\circ} \mathrm{C}\right)$ in which the average BAEP threshold was stable (Figure 12, A (inset)).

The I-II, II-III and III-IV interpeak latencies increased with decreasing temperature (Figure 12, B). III-IV interpeak latency responded differently to changes in temperature then was seen in the I-II and II-III interpeak latencies. All three of the interpeak latencies remained equal in the same temperature range as the BAEP threshold was stable $\left(20-26^{\circ} \mathrm{C}\right)$. The III-IV interpeak latency diverged from the earlier interpeak latencies at temperatures below $20^{\circ} \mathrm{C}$. Below $20^{\circ} \mathrm{C}$, III-IV interpeak latency increased at 

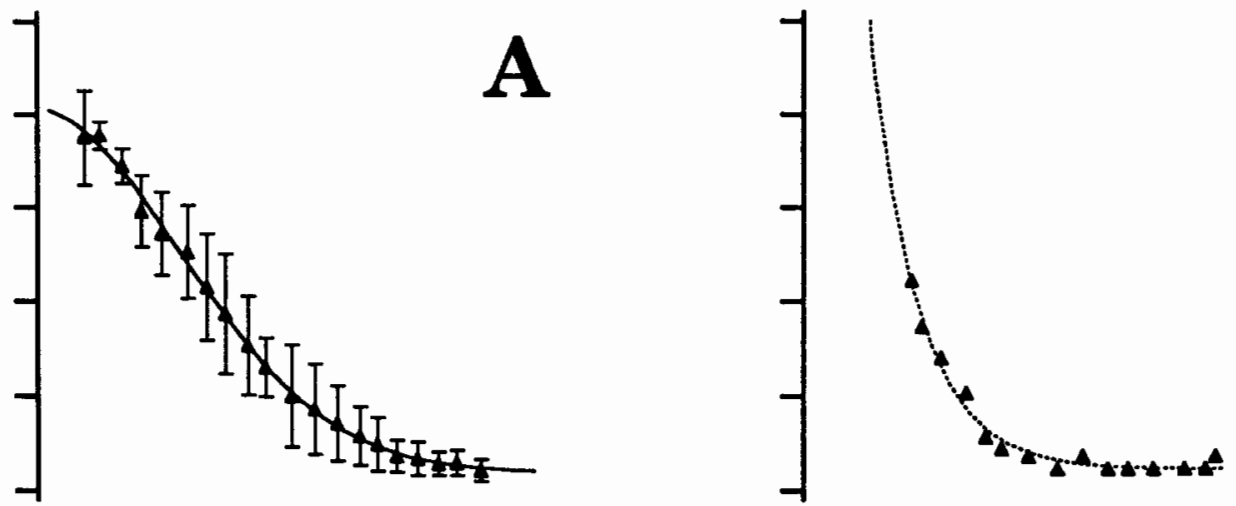

B
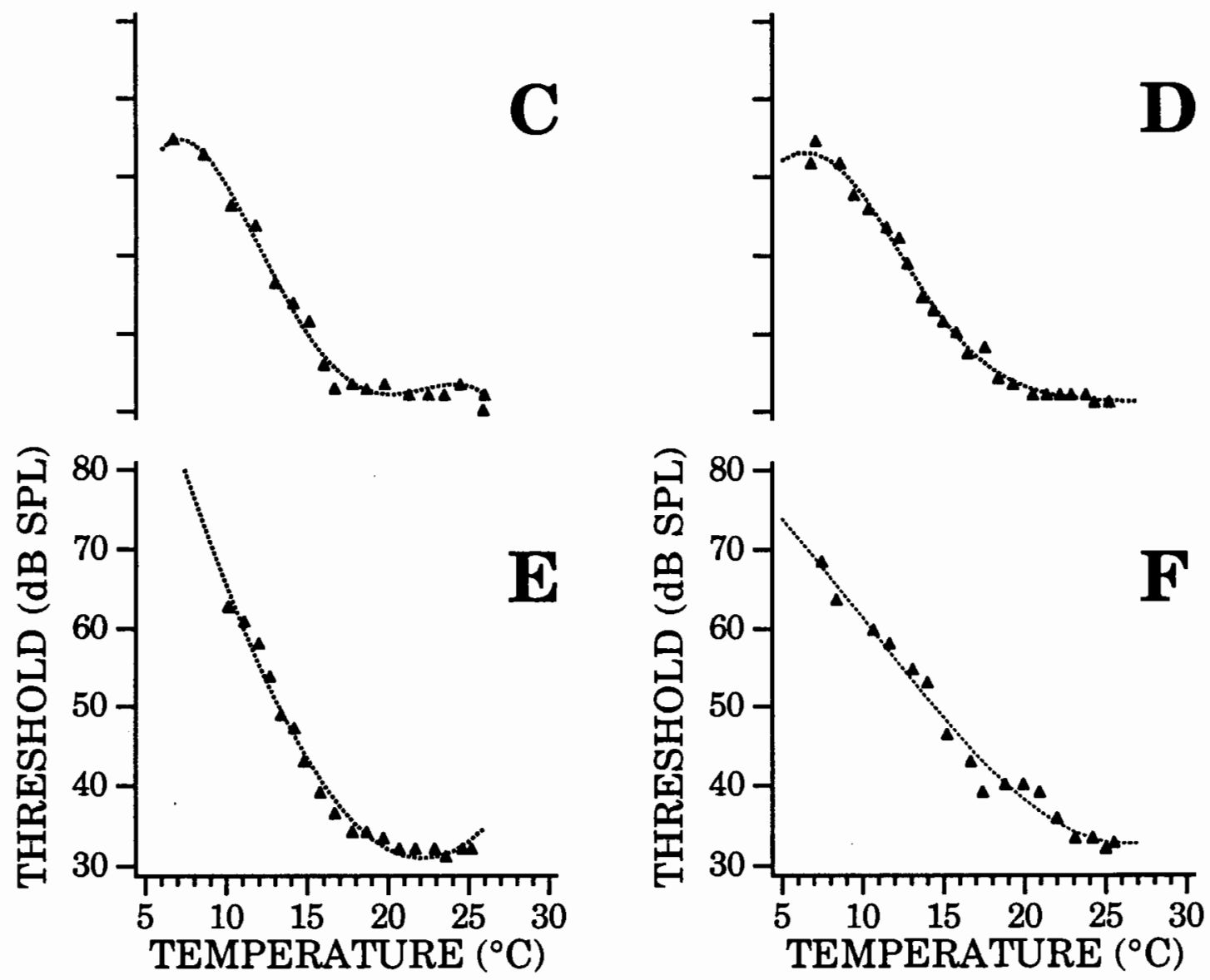

Figure 11. BAEP threshold response to changes in temperature.

(A) Average BAEP threshold for Rana pipiens acclimated to $21^{\circ} \mathrm{C}$ in response to a change in temperature $(n=9)$. Error bars indicate s.d. for the temperatures. Gaussian line fit to show trend with temperature. Note that average BAEP threshold was stable at temperatures above $20^{\circ} \mathrm{C}$. Below $20^{\circ} \mathrm{C}$, mean BAEP threshold increased markedly. (B-F) BAEP thresholds for five selected frogs acclimated to $21^{\circ} \mathrm{C}$ in response to a change in temperature. Each of the frogs showed the similiar effect of temperature on the BAEP threshold shown in A. 


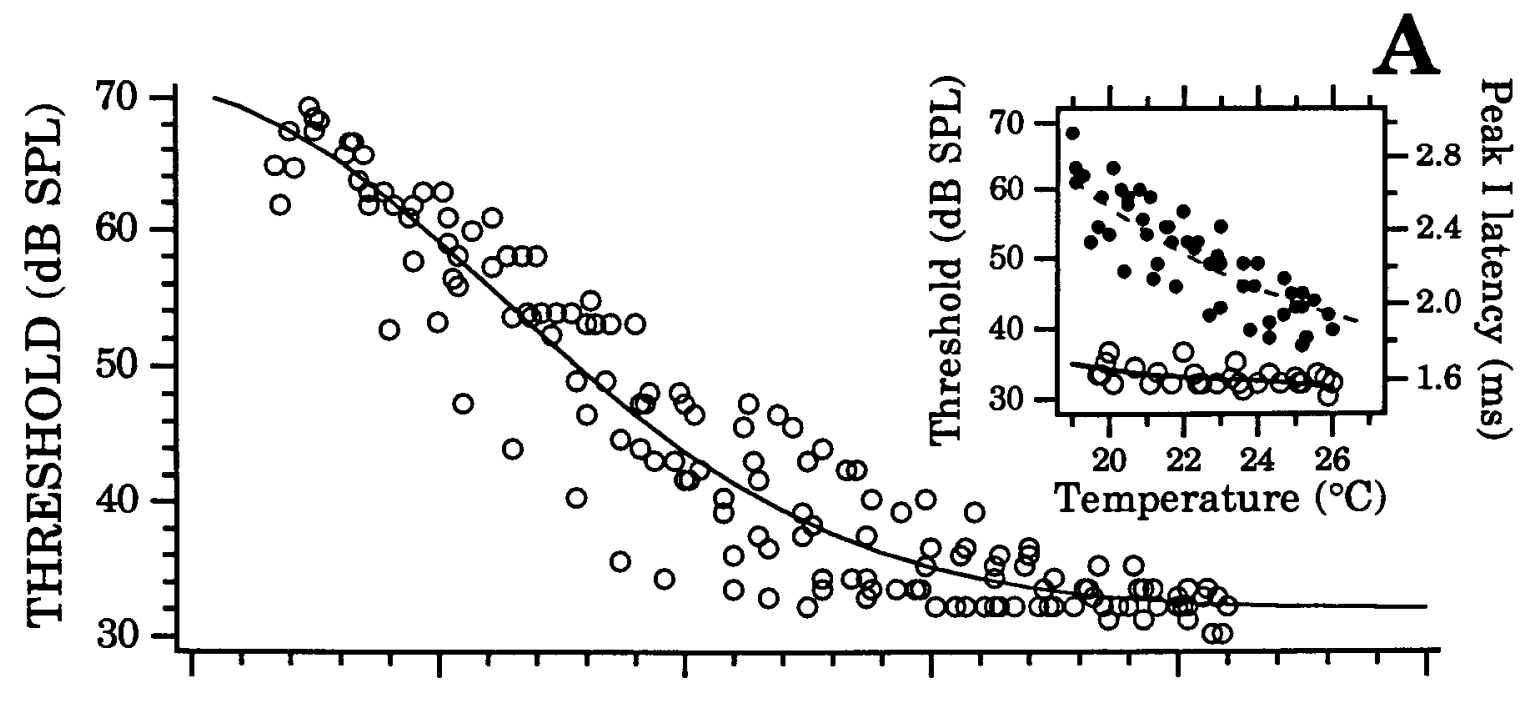

告

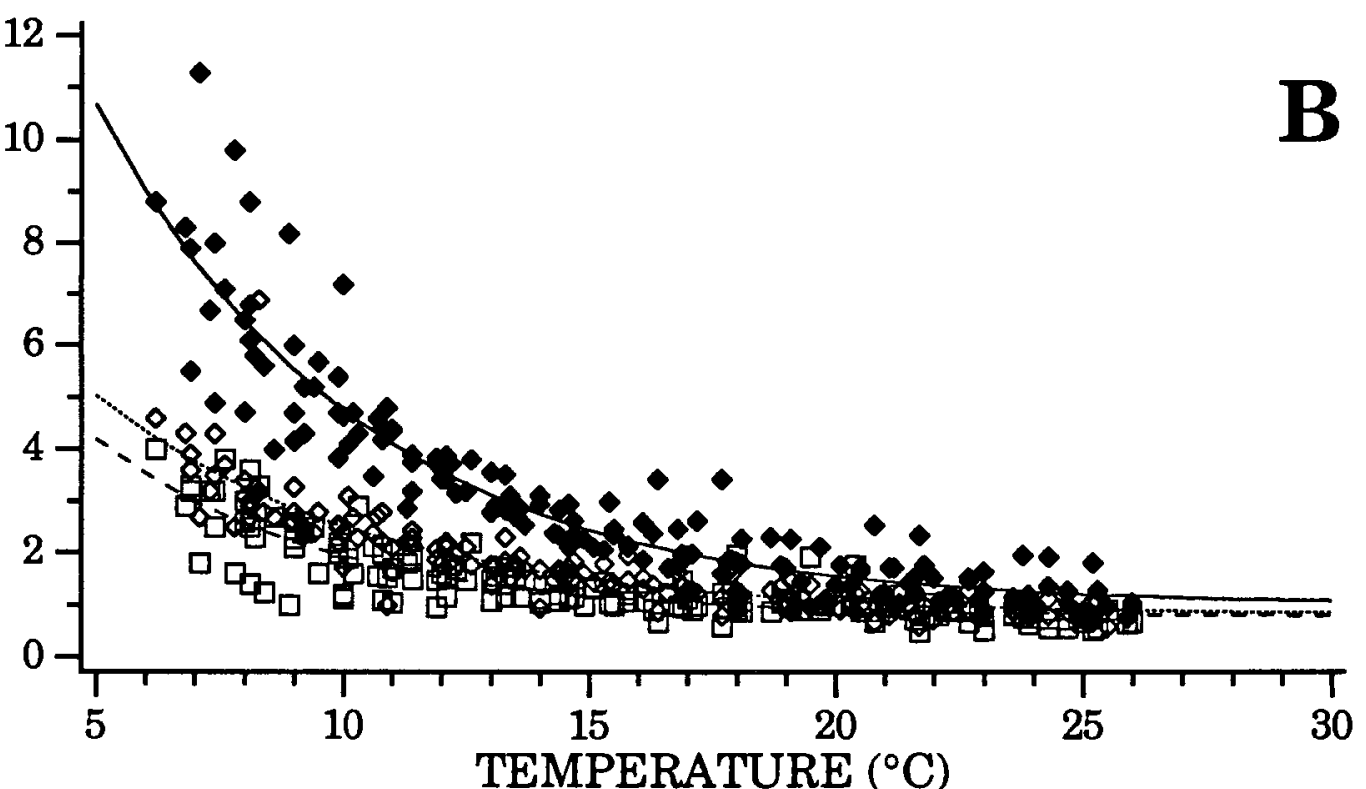

Figure 12. Comparison of changes in BAEP threshold, peak I latency, and three interpeak latencies with temperature. (A) Scatterplot showing the change in BAEP threshold with temperature for all frogs studied. A gaussian fit line is included to show trend. The inset is a comparison of the BAEP threshold (unfilled circles) and peak I latency (filled circles) change with temperature in the temperature range in which the BAEP threshold was stable. (B) Scatterplots showing the change in different interpeak latencies (I-II; unfilled diamonds, II-III; unfilled squares, III-IV; filled diamonds) with temperature. Exponential fit lines are included to show trend. The different interpeaks are equal in latency at temperatures above $20^{\circ} \mathrm{C}$. Below $20^{\circ} \mathrm{C}$, III-IV interpeak latency increased at a higher rate than the earlier interpeaks. I-II and II-III interpeak latencies increased at an average rate of 0.21 and $0.16 \mathrm{~ms} /{ }^{\circ} \mathrm{C}$, while III-IV interpeak latency increased at an average rate of $0.40 \mathrm{~ms} /{ }^{\circ} \mathrm{C}$. 
an average rate of $0.40 \mathrm{~ms} /{ }^{\circ} \mathrm{C}$, while I-II and II-III interpeak latencies increased at a rate of 0.21 and $0.16 \mathrm{~ms} /{ }^{\circ} \mathrm{C}$, respectively. The divergence of the III-IV interpeak latency from the earlier interpeak latencies at temperatures below $20^{\circ} \mathrm{C}$ was similar to the shift in the BAEP threshold in the same temperature range (Figure 13).

Peaks I and IV were used to analyze the effects of temperature on peak amplitude in $R$. pipiens, since they were the most stable of the four and they allowed us to compare the responses of the peripheral and central portions of the nervous system to changes in temperature. Peak I and IV amplitude data were normalized to the percent of maximum amplitude for each of the peaks . For each frog, the peak I and peak IV amplitudes were divided by the maximum peak I and peak IV amplitudes, respectively, attained by each frog at any temperature.

The amplitudes of peak I and IV responded slightly differently to temperature change (Figure 14). The amplitude of peak I increased as the frogs were warmed, reaching a maximum at $13^{\circ} \mathrm{C}$ and maintaining that maximum amplitude through about $19^{\circ} \mathrm{C}$. As the frogs were warmed above $19^{\circ} \mathrm{C}$, the amplitude of peak I decreased, but not to the amplitudes seen at the lower temperatures (Figure 14, A). The amplitude of peak IV increased as the frogs were warmed, also reaching a maximum around $13^{\circ} \mathrm{C}$. There was no apparent declining trend at higher temperatures, however (Figure 14, B). Peak I and IV amplitudes were highly variable and the description of trends were based on visual perception of the data.

The response of the peak amplitudes differed from the response of the BAEP threshold to the increase in temperature, even though BAEP threshold is based on the amplitude of the BAEP waveform and more specifically on the 


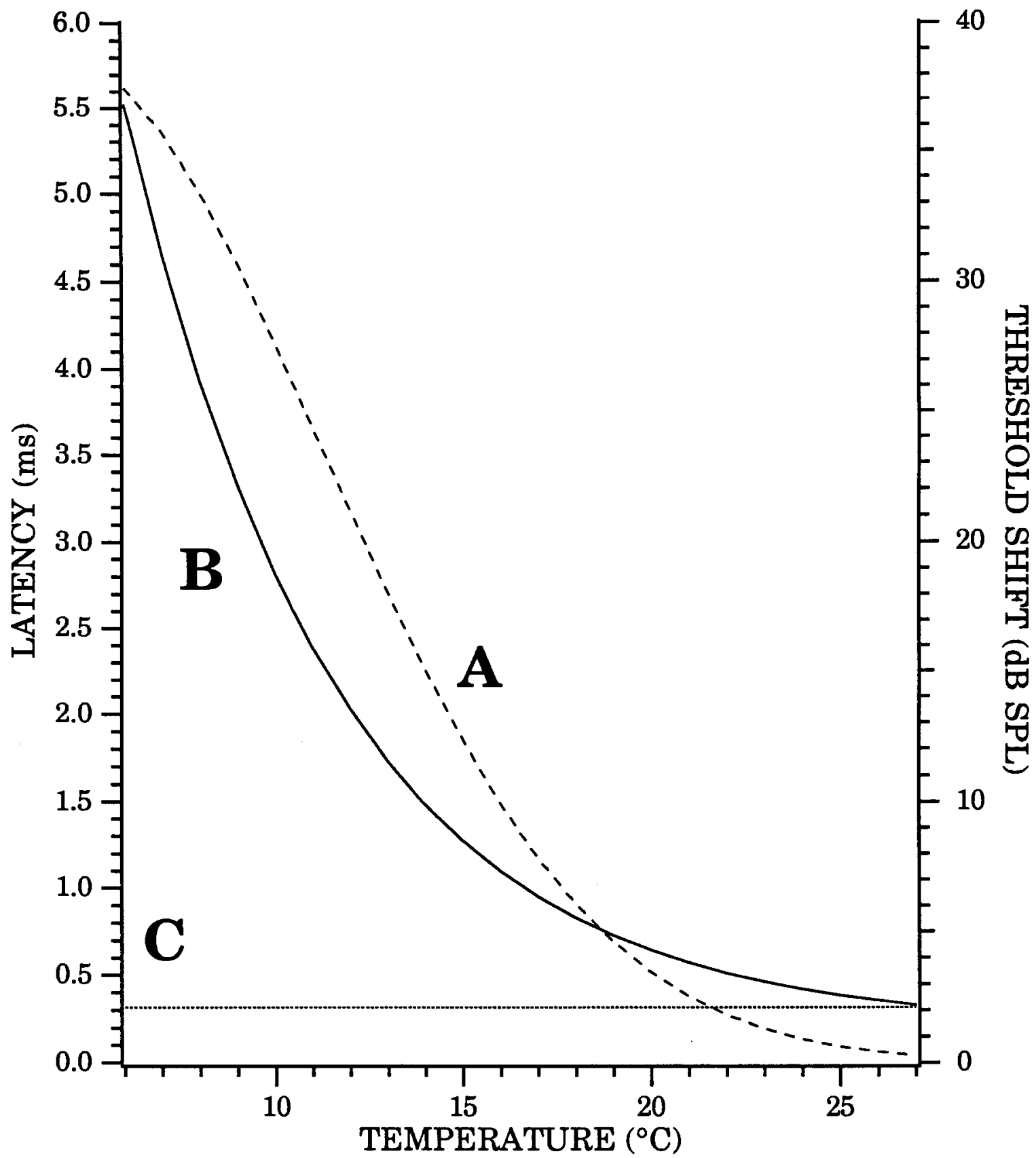

Figure 13. Temperature dependence of BAEP threshold shift compared with divergence of interpeak latencies. (A) BAEP threshold shift; dashed-line, (B) III-IV interpeak latency divergence from II-III interpeak latency; solid-line, (C) I-II interpeak latency divergence from II-III interpeak latency; dotted-line. A gaussian curve fit was applied to both interpeak divergence and BAEP threshold shift data to compare the trends. Note that the change in the BAEP threshold shift with temperature was similar to that seen in the divergence of III-IV interpeak latency from the earlier interpeak latencies. 

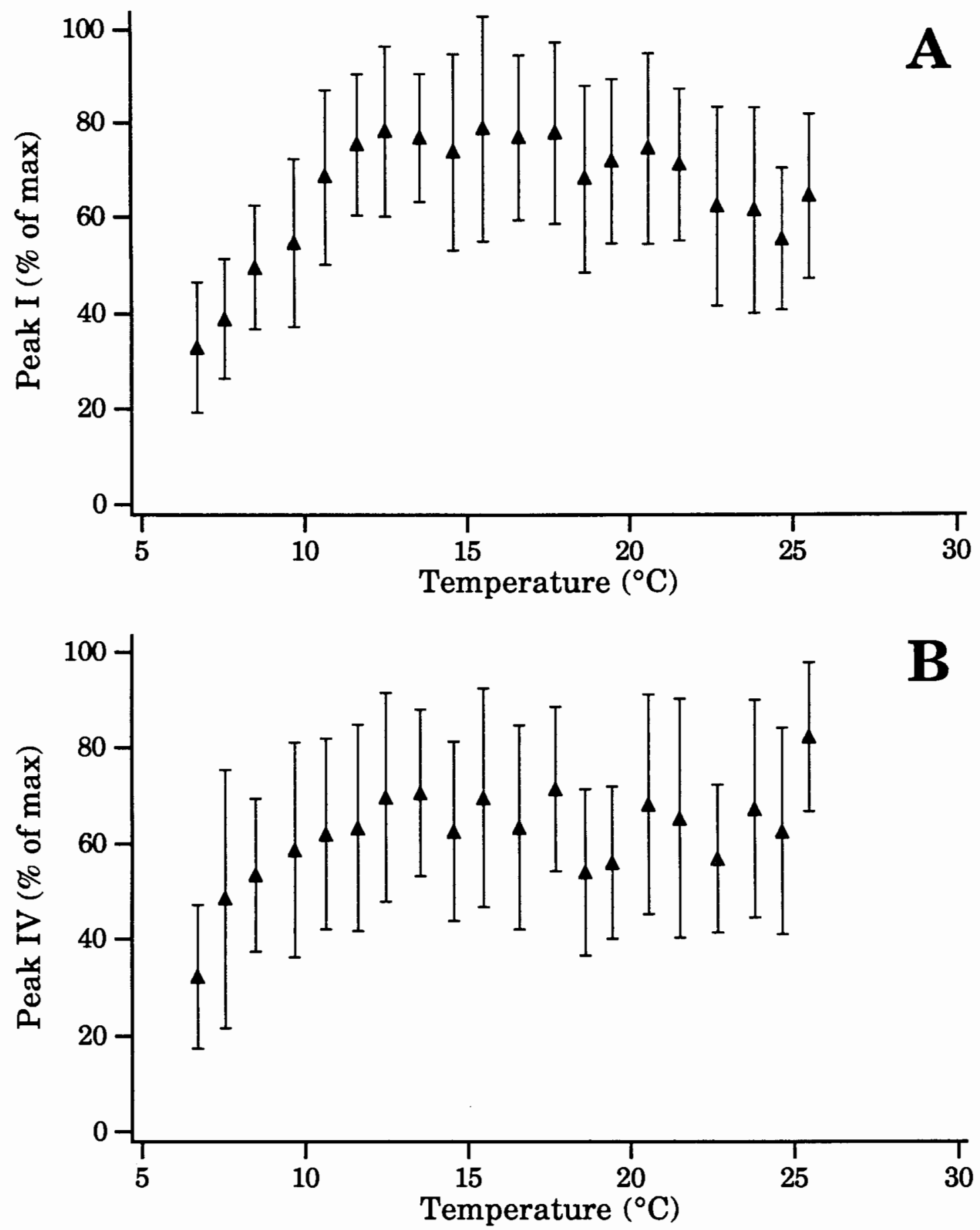

Figure 14. Peak I (A) and IV (B) amplitudes for $R$. pipiens in response to changes in temperature. Amplitude data were normalized to percent of maximum amplitude. Points are means and ( \pm s.d.). 
presence or absence of peak IV. Peak IV amplitude was stable in the higher temperatures as was the BAEP threshold. But as the temperature was lowered below $20^{\circ} \mathrm{C}$, the BAEP threshold increased while the amplitude of peak IV remained stable until the temperature had dropped below $13^{\circ} \mathrm{C}$, where it began to decrease in amplitude. This discrepancy could be a result of the difference in minimum sound level ( $75 \mathrm{~dB}$ SPL) necessary for the saturation of peak IV amplitude and the test sound level (87 dB SPL). A temperature-dependent decrease in the peak IV amplitude would not be detected, using a test sound level of $87 \mathrm{~dB}$ SPL) until the temperature effect on peak IV amplitude overcame the excess saturation of peak IV.

\section{Effects of acclimation on the BAEP}

Nineteen Rana pipiens were acclimated to $14^{\circ} \mathrm{C}$ (ten frogs) and $21^{\circ} \mathrm{C}$ (nine frogs) for at least three weeks. Following the cooling of each acclimated frog to its individual-dependent minimum temperature, frogs were transiently warmed from 6 to $26^{\circ} \mathrm{C}$ at a rate of $0.2^{\circ} \mathrm{C} / \mathrm{min}$. During transient warming, BAEPs were recorded in response to $87 \mathrm{~dB}$ SPL acoustic clicks, and BAEP threshold determined. Acoustic measurements were repeated for every $1{ }^{\circ} \mathrm{C}$ temperature change. Waveform morphology was similar between the different acclimated frogs.

BAEP threshold response to increasing temperature was different for frog groups acclimated to 14 and $21^{\circ} \mathrm{C}$ (Figure 15). While both groups followed the same trend of having a relatively stable threshold above $20^{\circ} \mathrm{C}$ and a monotonically increasing threshold as temperature was lowered below $20^{\circ} \mathrm{C}$, the BAEP threshold for the $14^{\circ} \mathrm{C}$ acclimated frogs was higher at temperatures above $15{ }^{\circ} \mathrm{C}$ compared with frogs acclimated to $21^{\circ} \mathrm{C}$. The difference between BAEP threshold for the two groups increased as the 

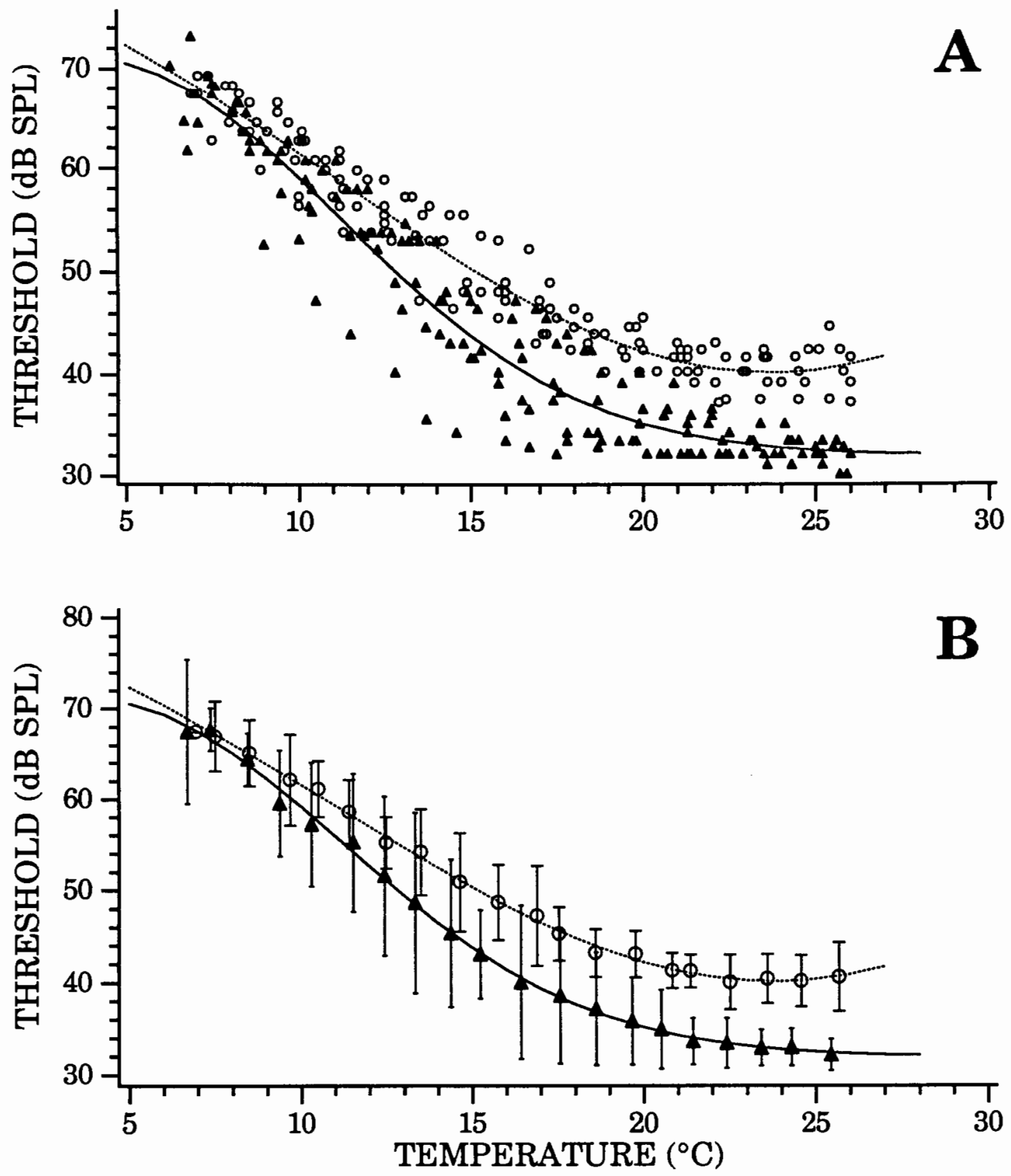

Figure 15. BAEP threshold response vs. temperature for frogs acclimated to 14 and $21^{\circ} \mathrm{C}$. (A) Scatterplot of the change in BAEP threshold to an increase in temperature. Unfilled circles: frogs acclimated to $14^{\circ} \mathrm{C}$. Filled triangles: frogs acclimated to $21^{\circ} \mathrm{C}$. A best fit line (gaussian) was plotted to show the different trends of BAEP threshold response to an increase in temperature for the different acclimated frogs. (B) Plot showing the change in average BAEP threshold \pm (95\% confidence intervals) for frogs acclimated to 14 and $21^{\circ} \mathrm{C}$ in response to an increase in temperature. The lines are the same as those computed for $\mathrm{A}$. 
temperature was increased above $15^{\circ} \mathrm{C}$ with the maximum difference (8-10 $\mathrm{dB}$ SPL) occurring in the relatively stable region above $20^{\circ} \mathrm{C}$.

There was also a difference in the peak I latency for frogs acclimated to 14 and $21^{\circ} \mathrm{C}$ (Figure $16 \mathrm{~A}$ ). At all test temperatures above $15^{\circ} \mathrm{C}$; with the peak I latencies for frogs acclimated to $14^{\circ} \mathrm{C}$ were higher than for frogs acclimated to $21^{\circ} \mathrm{C}$. The difference between the peak I latency became less apparent as the temperature of the frogs were decreased. The difference in the BAEP thresholds disappeared in a similar fashion.

The III-IV interpeak latency for $21^{\circ} \mathrm{C}$ and $14{ }^{\circ} \mathrm{C}$ acclimated frogs was the same, and remained the same for transient warming. This suggests that central auditory pathways in $14{ }^{\circ} \mathrm{C}$ acclimated frogs perform at the same level as $21{ }^{\circ} \mathrm{C}$ acclimated frogs (Figure $\left.16 \mathrm{~B}-\mathrm{D}\right)$.

Since both the BAEP threshold and the peak I latency showed a shift due to temperature acclimation, I decided to compare the measured threshold difference between the 14 and $21^{\circ} \mathrm{C}$ acclimated frogs and the expected threshold difference corresponding to the peak I latency difference between the two groups of frogs. The purpose of this analysis was to see if the peak I latency difference between the $14^{\circ} \mathrm{C}$ and $21^{\circ} \mathrm{C}$ acclimated frogs could account for the observed threshold shift. If so, a solely peripheral mechanism is sufficient to account for the acclimation-induced threshold shift.

I determined the expected BAEP threshold shift from the peak I latency difference for six temperature ranges (Figure $17 \mathrm{C}$ ) by first determining a corrected test sound level. The corrected test sound level was the standard test sound level (87 dB SPL) minus the threshold shift which had occurred due to the transient cooling of the frog, relative to the ambient $\left(21^{\circ} \mathrm{C}\right)$ threshold. For example, a frog at $19^{\circ} \mathrm{C}$ has a threshold shift of $2.0 \mathrm{~dB}$ 

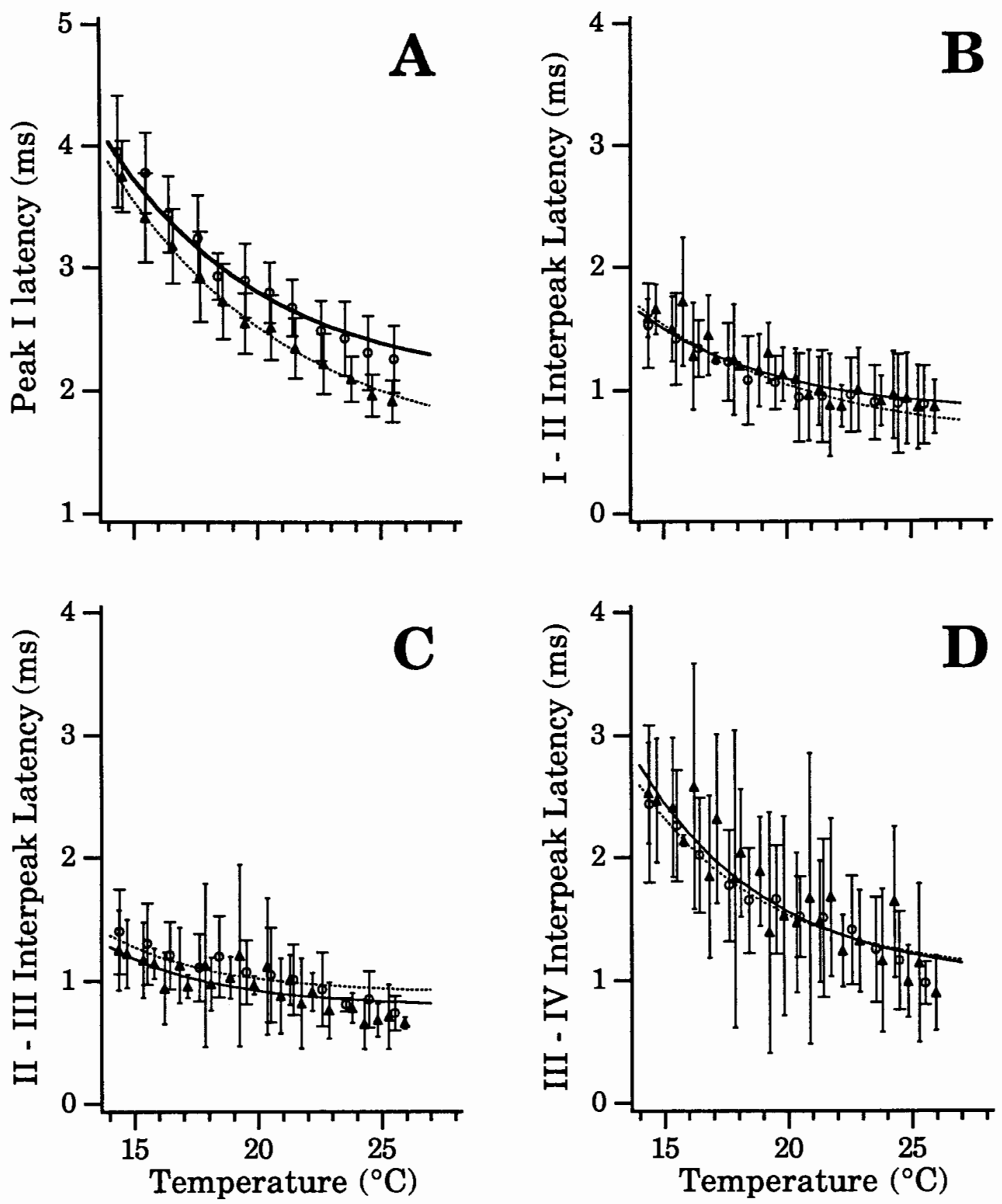

Figure 16. Peak I and interpeak (I-II, II-III and III-IV) latency change to an increase in temperature for $R$. pipiens acclimated to 14 and $21{ }^{\circ} \mathrm{C}$. Unfilled circles; frogs acclimated to $14^{\circ} \mathrm{C}$. Filled triangles; frogs acclimated to $21^{\circ} \mathrm{C}$. Points are means $\pm 95 \%$ confidence intervals. (A) Peak I latency. A best line fit (exponential) plotted to show trends. (B) I-II interpeak latency comparison. (C) II-III interpeak latency comparison. (D) III-IV interpeak comparison. 


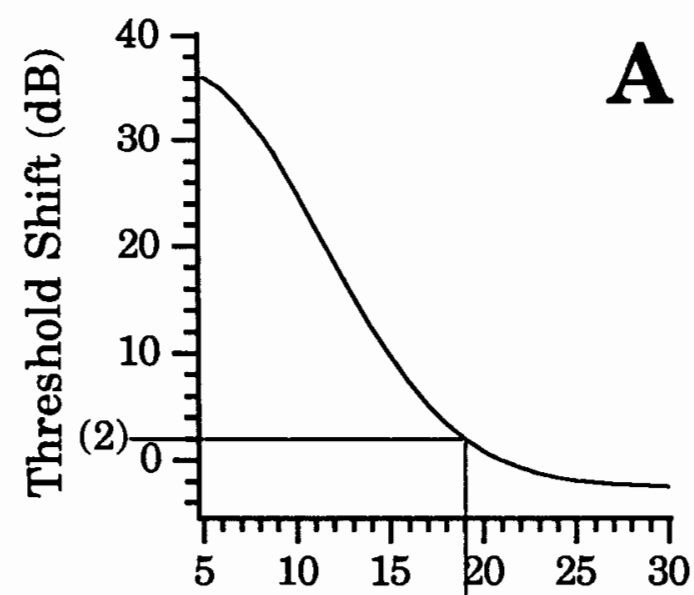

(1)

Temperature $\left({ }^{\circ} \mathrm{C}\right)$

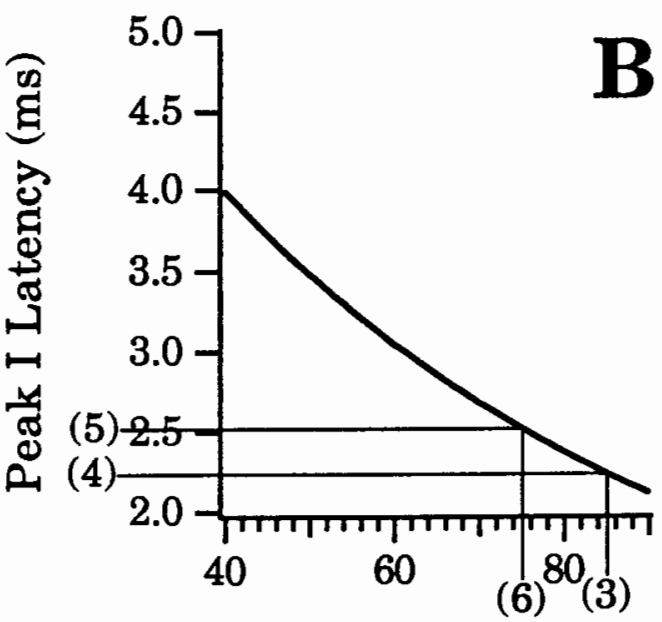

Sound Level (dB SPL)

Expected Threshold Shift

$\square$ Measured Threshold Shift

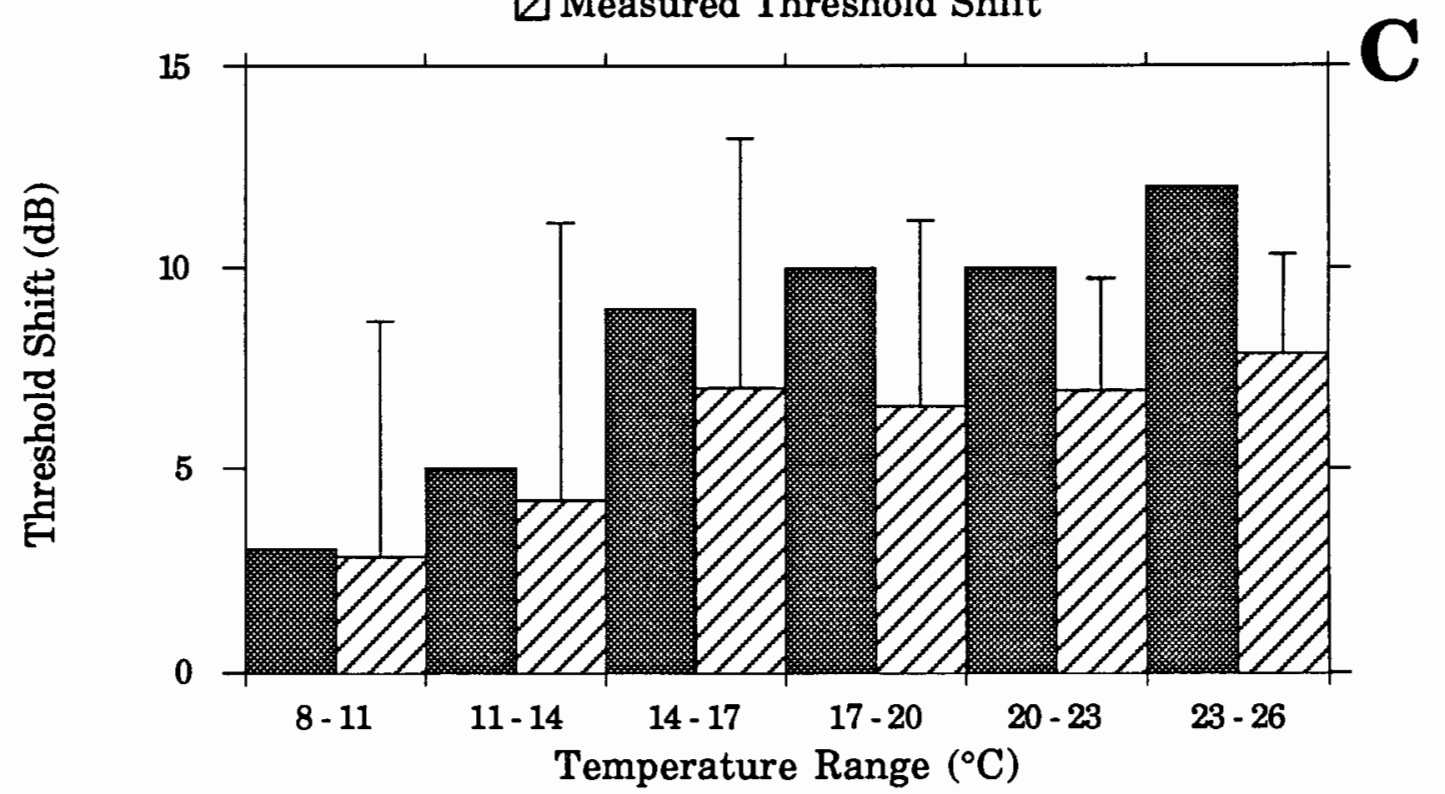

Figure 17. A comparison of the average expected BAEP threshold shift calculated from the peak I latency difference between $R$. pipiens acclimated to 14 and $21^{\circ} \mathrm{C}$ and the average BAEP threshold difference for the same frogs at six different ranges of test temperatures.

(A) BAEP threshold shift versus temperature for frogs acclimated to $21^{\circ} \mathrm{C}$. (B) Peak I latency versus intensity for $R$. pipiens acclimated to $21^{\circ} \mathrm{C}$ (from Fig. 5A). I used this peak I latency versus intensity relationship to calculated the expected BAEP threshold shift between frogs acclimated at 14 and $21^{\circ} \mathrm{C}$. (C) A comparison of the expected BAEP threshold shift and the measured threshold shift between frogs acclimated at 14 and $21^{\circ} \mathrm{C}$ for seven temperature ranges (See results for explanation). 95\% confidence intervals are shown for the measured threshold shift. 
relative to ambient threshold (Figure 17, A.1 and A.2). This threshold shift was then subtracted from the standard test intensity ( $87 \mathrm{~dB}$ SPL) giving a corrected test intensity of $85 \mathrm{~dB}$ SPL. This correction was done to place us on the proper portion of the peak I latency versus intensity curve (Figure 17, B) when determining the expected threshold shift, since a frog at $21^{\circ} \mathrm{C}$ perceives the sound level of an acoustic stimulus differently than a frog at another temperature, based on BAEP threshold. Using the latency-sound level curve in figure $17 \mathrm{~B}$, I then determined the corresponding corrected peak I latency for the corrected test sound level (Figure 17, B.4 $=2.24 \mathrm{~ms}$ ). Based on the test temperature $\left(19^{\circ} \mathrm{C}\right)$, I next determined the peak I latencies for frogs acclimated to 14 and $21^{\circ} \mathrm{C}$, using the fitted curves in figure $16 \mathrm{~A}$, and estimated the difference between the two peak I latencies $(0.28 \mathrm{~ms})$. This difference was then added to the corrected peak I latency (B.4) to calculate the expected peak I latency $(B .5=2.52 \mathrm{~ms})$. Again using the latency-sound level curve, I determined the corresponding sound level $(B .6=75.0 \mathrm{~dB} \mathrm{SPL})$ necessary to create the expected peak I latency. I then subtracted B.6 from B.3 to determine the expected threshold shift $(10 \mathrm{~dB})$ based on the differences in the peak I latencies.

I compared the expected BAEP threshold shift based on the peak I latency difference between frogs acclimated to 14 and $21^{\circ} \mathrm{C}$ for six arbitrary temperature ranges, to the experimentally determined BAEP threshold shifts (Figure 17, C). The expected BAEP threshold shifts were in all cases larger than the measured threshold shifts. Thus while this method does not predict the exact threshold shift observed, it does indicate that there is sufficient acclimation-induced latency change to correlate peak I latency with threshold shift. 
The amplitudes of peaks I and IV were analyzed for both acclimation temperature groups (Figure 18, A and B). Even though there was a shift in BAEP thresholds for frogs acclimated to $21^{\circ} \mathrm{C}$ compared with frogs acclimated to $14^{\circ} \mathrm{C}$, there was no difference in the amplitudes of peaks I or IV between the two groups.

I monitored the BAEP threshold and peak I latency as the $14^{\circ} \mathrm{C}$ frogs were allowed to reacclimate to room temperature $\left(21^{\circ} \mathrm{C}\right)$. After 3-5 days, BAEP threshold and peak I latency recovered to ambient temperature levels, which would indicate that the three week time period used to acclimate frogs to either test temperature was sufficient to insure temperature acclimation.

\section{DISCUSSION}

\section{Temperature effects on the BAEP}

Since anurans are ectotherms, they are incapable of metabolically maintaining a specific body temperature which is different from their environmental temperature. The functional capabilities of the auditory system and indeed of nervous systems in general are strongly dependent on temperature (Eatock and Manley, 1981; Marsh, et al., 1984). We have seen temperature-induced functional impairments of both peripheral and central auditory systems at lower temperatures using the BAEP of Rana pipiens.

In general, the effect of temperature on the auditory system seems to be both in the inner ear and more centrally (Campbell, 1969; Werner, 1976). The characteristic frequency (CF) of tuning curves for nerve fibers leaving the sensory epithelium of the inner ear decreases as temperature decreases for the ectotherms, the tokay gecko and several species of anurans (Eatock and Manley, 1981; Hubl and Schneider, 1979a; van Dijk, et al., 1990). For 

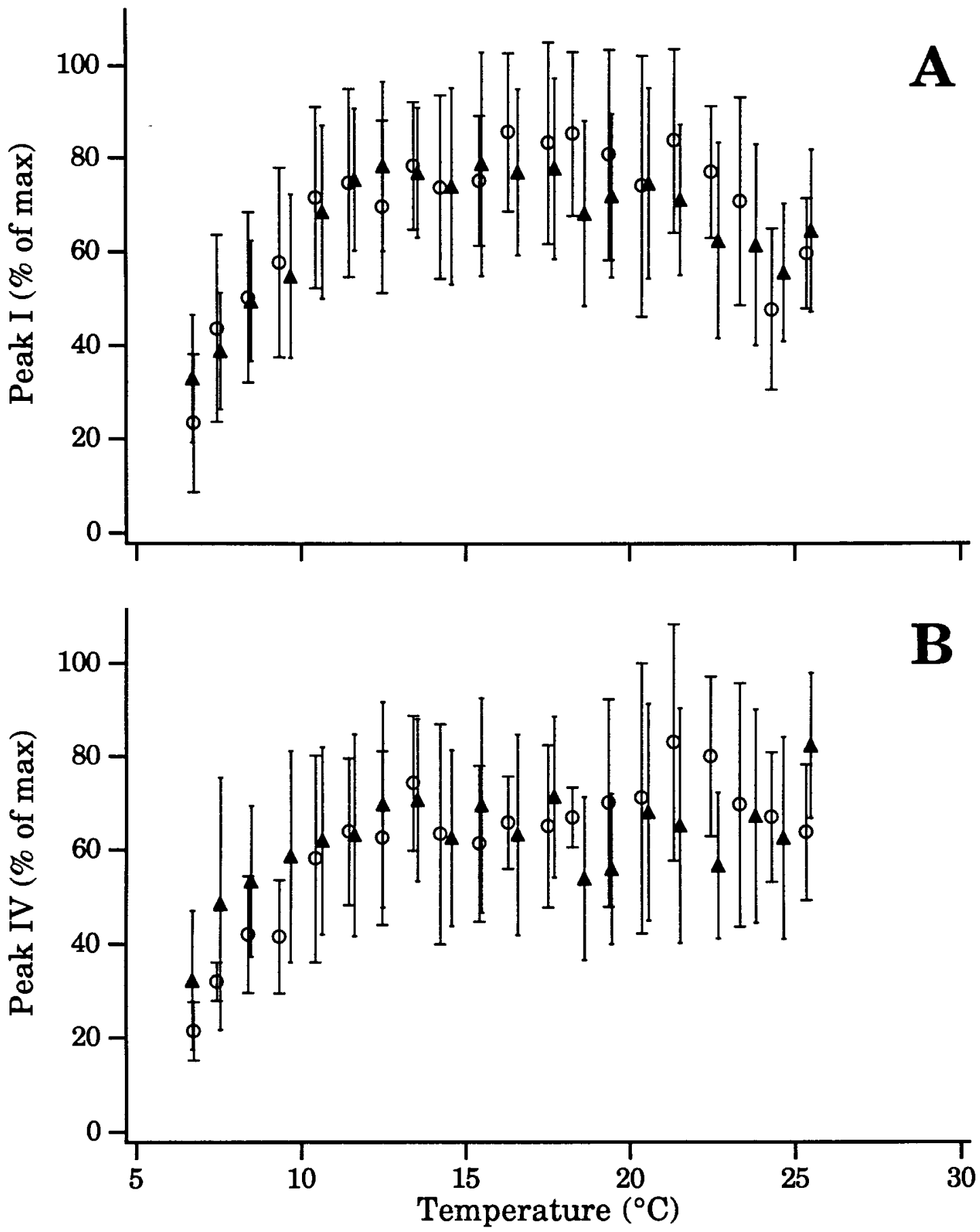

Figure 18. Peak I (A) and IV (B) amplitudes to change in temperature for $R$. pipiens acclimated to 14 and $21^{\circ} \mathrm{C}$. Values normalized to percent of maximum peak amplitude. Unfilled circles; frogs acclimated to $14^{\circ} \mathrm{C}$. Filled triangles; frogs acclimated to $21^{\circ} \mathrm{C}$. Points are means $( \pm$ s.d.). These plots followed the same plotting guidelines and expressed the same trends expressed in figure 13. 
anurans, temperature mainly effects the amphibian papilla with little effect on the basilar papilla (Mohneke and Schneider, 1979). Characteristic frequency (CF) thresholds and latencies for midbrain nuclei (TS) sensitive to frequencies derived from the amphibian papilla, increase with decreasing temperature. Since the BAEP is strongly affected by changes in temperature, this would suggest that the amphibian papilla contributes to the BAEP more than the basilar papilla.

Other animals, in particular the alligator and the human, show very little increase in the peak I latency as temperature is decreased, instead most of the latency shifts occur between peaks (Rosenblum, et al., 1985; Strain, et al., 1987). The sensory epithelium of mammals and reptiles differ from the lower vertebrates, which may result in the different responses of the peak I latencies to temperature.

The increase in interpeak latency of the BAEP in response to a decrease in temperature is most likely a result of a decrease in action potential (AP) conduction velocity and a decrease in synaptic transmission efficacy. Over a similar temperature range as was used with Rana pipiens in the present study $\left(26^{\circ} \mathrm{C}-6{ }^{\circ} \mathrm{C}\right.$ ), the AP conduction velocity for Bufo marinus decreases from $30 \mathrm{~m} / \mathrm{s}$ to $10 \mathrm{~m} / \mathrm{s}$ (Macdonald, 1981). Synaptic transmission is limited by channel conductance, vesicle migration, diffusion and receptor binding, all chemical processes expected to have temperature dependencies. The rate of chemical reactions involved in complex biological phenomena, such as muscle contraction, nerve impulse propagation, and respiration typically double every $10^{\circ} \mathrm{C}\left(\mathrm{Q}_{10}=2\right)$ (Hartenstein, 1972).

Along with decreased AP conduction velocity, a possible reason for the increase in peak I latency during cooling is the delay in signal transduction in 
the sensory epithelium of the papillae. In saccular hair cells of bull frogs, there is a longer time course for ionic flow through transduction channels located in the stereocilia under hypothermic conditions (Corey and Hudspeth, 1983). This slowing of the ionic flow could result in a delay in the AP generation in the VIIIth nerve.

Although $Q_{10}$ 's may be used to describe the temperature effects on relatively simple physiological processes (AP conduction and synaptic transmission), complex processes tend to have a nonlinear response to changes in temperature. For example, in the ectotherm, Dipsosaurus dorsalis, digestive efficiency, endurance, metabolism and relative hearing respond nonlinearly with changes in temperature (Huey, 1982).

BAEP threshold depends on peripheral and central auditory system processes, which are, evidently, sufficiently complex to result in a nonlinear response to changes in temperature. Indeed, BAEP threshold is primarily dependent on the amplitude of peak $\mathrm{IV}$, which is generated in a region of the auditory system where complex processing of the neural information is beginning to occur (Covey, et al., 1991; Feng, et al., 1991; Hall and Feng, 1991). With the increase in synaptic activity associated with integrating centers of the brain, there could be an increase in the probability of synaptic transmission dysfunction as a result of temperature decrement, which could have a profound effect on auditory sensitivity.

BAEP threshold is determined by the presence or absence of the BAEP peaks. The immediate conclusion would be that the BAEP threshold would be dependent on the amplitudes of the peaks. This does not seem to be the case, however, since we observed that BAEP threshold increased as the temperature dropped below $20^{\circ} \mathrm{C}$ while the amplitudes of peaks I and IV, in 
response to an $87 \mathrm{~dB}$ SPL acoustic stimulus, remained stable until the temperature dropped below $13^{\circ} \mathrm{C}$.

I suspect that the early shift in the BAEP threshold is a result of a breakdown in complex neural processing areas represented by peaks III and IV. Between 20 and $26^{\circ} \mathrm{C}$, all three interpeaks were equal in latency and the BAEP threshold was stable. Once the temperature of the frog had dropped below $20^{\circ} \mathrm{C}$, III-IV interpeak latency increased at a higher rate than the earlier interpeaks, and BAEP threshold began to increase. Since III-IV interpeak latency increased at a higher rate than the earlier interpeak latencies, this suggests that the signal being conducted from the peak III to IV neural generators was reduced in strength, perhaps due to loss of synchrony. If there was a reduction in the signal strength from peak III to peak $I V$, one would expect a corresponding reduction in the peak IV amplitude. We did not see a reduction in the peak IV amplitude until the temperature of the animal was decreased below $\sim 13^{\circ} \mathrm{C}$. Since peak IV amplitude saturates at stimulus sound levels above $75 \mathrm{~dB}$ SPL, and I used a test signal of $87 \mathrm{~dB}$ SPL, a reduction in the peak IV amplitude would not be apparent until the temperature was low enough to attenuate the signal getting to the peak $\mathrm{NV}$ generator below $75 \mathrm{~dB}$ SPL. Thus the apparently anomalous maintenance of peak IV amplitude between 13 and $20{ }^{\circ} \mathrm{C}$ is probably due simply to an excess of test sound level.

Recently, Attias (1991) (Attias, et al., 1991)has localized the more temperature sensitive nuclei in the auditory pathway of man using BAEPs. The peaks which corresponded to the sensitive nuclei (III and IV) decreased in latency with increasing temperature. These peaks may correspond to 
neural generators which are analogous to those peaks which are temperature sensitive in $R$. pipiens.

\section{Effects of acclimation on the BAEP}

The only difference in the BAEP response to changes in temperature for frogs acclimated to $14^{\circ} \mathrm{C}$ and to $21^{\circ} \mathrm{C}$ was in peak I latency and BAEP threshold. There was no difference between the interpeak latencies and amplitudes for either sets of frogs.

Studies have shown that nerves adapted to cooler temperatures have decreased action potential velocities over nerves acclimated to warmer temperatures (Bishop and Gordon, 1967; Macdonald, 1981). The decreased conduction velocities of the cooler nerves may be a result of the regulation of membrane fluidity. Cold-adapted nerves have higher proportions of unsaturated fatty acids which are less viscous than fatty acids comprising membrane lipids of warm-adapted animals. Decreased membrane fluidity can have an influence on electrically sensitive ion channels (Gage, 1976). Thus the increase in the BAEP threshold for $R$. pipiens acclimated to $14^{\circ} \mathrm{C}$ may be a result of decreased sensitivity of the sensory epithelium in response to decrease in the membrane fluidity which would effect voltage sensitive ion channels. The decreased sensitivity of the peripheral sensory epithelium would result in a peak I latency shift. Since prediction of the BAEP acclimation threshold shift using the peak I latency difference is not exact, most likely a combination of factors mentioned above (sensory epithelium ion channel effects, decreased action potential velocity, and loss of synchrony in more complex CNS sensory nuclei) affects threshold shift (figure 17).

Changes in the membrane fluidity would mainly contribute to acclimation effects rather than transient temperature change effects. The 
time course for regulation of unsaturated fatty acids in the membrane is too long (days) to respond to transient changes in temperature (Macdonald, 1981).

The difference in BAEP threshold due to acclimation may have a significant impact on anuran ecology. Sound pressure decreases $6 \mathrm{~dB}$ for every doubling of distance. For example, if the level of a sound is $60 \mathrm{~dB}$ SPL at a distance of $10 \mathrm{~m}$, the sound will be $54 \mathrm{~dB}$ SPL at a distance of $20 \mathrm{~m}$. Between 20 and $26^{\circ} \mathrm{C}$ body temperature, a Rana pipiens acclimated to $14{ }^{\circ} \mathrm{C}$ is 8-10 $\mathrm{dB}$ less sensitive then $R$. pipiens acclimated to $21^{\circ} \mathrm{C}$, and therefore would have to be twice as close to a sound source to have equal detection. Thus acclimation temperature would influence the spatial range in which a Rana pipiens perceives its surrounding acoustic environment. At sustained temperatures of $14^{\circ} \mathrm{C}$, then, the frog would suffer a decrease in the probability of detecting an advertisement call and an increase in the probability of becoming a prey item. Although $R$. pipiens are able to acclimate to lower temperatures, they are not as sensitive as seen in the temperature range in which the animal is functioning at peak performance.

The range of temperatures in which the anuran auditory system is the most sensitive seems to be the preferred range for activity as well, as seen in other animals (Campbell, 1969). The peak performance capacity for Rana pipiens was attained in the temperature range between 20 and $29^{\circ} \mathrm{C}$ (Putnam and Bennett, 1981). When testing the auditory sensitivity of Rana pipiens, using the BAEP, I found that the temperature range with the greatest auditory sensitivity was 20 to $26^{\circ} \mathrm{C}$, which matches the range for peak performance. Similarly, the acclimation temperature of an anuran 
would have to be in the temperature range for peak performance capacity for the greatest auditory sensitivity. 
CHAPTER III

\section{EFFECTS OF DEHYDRATION AND HYPERNATREMIA ON THE BRAINSTEM AUDITORY EVOKED POTENTIAL OF THREE SPECIES OF ANURANS}

\section{INTRODUCTION}

The effects of dehydration, hypernatremia and hyponatremia on vertebrates have been heavily studied (Dodge, et al., 1962; Sotos, et al., 1960; Star, 1990). Changes in osmolality of body fluids are associated with many clinical conditions, including Turp syndrome, Diabetes mellitus and dialysis disequilibrium syndrome (Andrew, 1991).

There have been many studies on the effects of dehydration on various aspects of anuran anatomy and physiology (Hillman, 1980; Hillman, 1987; Krakauer, 1970; Shoemaker, 1964; Thorson and Svihla, 1943; Thorson, 1955). Anurans are excellent models for studying dehydration and hypernatremia because of their susceptibility to water loss. The skin of the anuran is unable to significantly prevent water from diffusing in either direction and an anuran may cycle up to $22 \%$ of its body mass in water per day (Gatten, 1987).

The tissues and organs of the frog respond differently to dehydration. As Rana pipiens are dehydrated such that they lose $43 \%$ of their original body mass in water, the brain only loses $7.8 \%$ of its original body mass while the blood loses up to $81 \%$ (Smith and Jackson, 1931). It is thought the brain prevents great water loss by uptake of ions from the blood $\left(\mathrm{K}^{+}\right)$and from the 
cerebral spinal fluid $\left(\mathrm{Na}^{+}\right.$and $\left.\mathrm{Cl}^{-}\right)$, thus keeping the water in the cells of the brain through simple diffusion (Star, 1990).

Since frogs are unable to prevent evaporative water loss, they have developed the means to store excess water in the bladder to be recovered by diffusion as needed (Thorson, 1955). Associated with dehydration in amphibians is a corresponding increase in various electrolyte concentrations, e.g., $\mathrm{Na}+, \mathrm{K}^{+}$, and $\mathrm{Ca}^{2+}$ (Alvarado, 1972; Hillman, 1978; Hillman, 1982). A specific increase in sodium is termed hypernatremia. With an increase in the osmolality of the blood in dehydrated individuals, water will move from the bladder back into the blood stream.

There are two physiological factors which correlate with the increasing levels of dehydration (Hillman, 1980). As the frog is dehydrated, the blood volume decreases resulting in increased blood viscosity, which reduces the capability of the circulatory system to deliver oxygen to the tissues. The second is that the $\mathrm{Na}+$ levels in the plasma and the CSF increases proportionally with increased water loss (Hillman, 1988).

Frogs and toads have a remarkable tolerance to hypernatremia as a result of increased dehydration levels. Mammals can tolerate only 30 to 60 $\mathrm{mM}$ increases in plasma sodium (Andrew, 1991) before spasms, nystagmus and finally death occurs (Arieff and Guisado, 1976; Star, 1990). Anurans are able to tolerate increases in the plasma sodium from $80 \mathrm{mM}$ for Xenopus laevis (Hillman, 1980) to $200 \mathrm{mM}$ for Bufo marinus (Krakauer, 1970). These increases in plasma sodium levels are equivalent to water loss of $33.8 \%$ to $52.6 \%$ of original body mass.

Electroencephalograms (EEGs) recorded from hypernatremic mammals show no changes in brain waves until the osmolality of the body 
fluids reached lethal levels, at which point the voltage level of the EEG decreases (Sotos, et al., 1960). Increased osmolar concentrations have no effect on the resting membrane potential or on the initiation of action potentials (Andrew, 1991).

In this study, I attempted to determine if the increased levels of dehydration and hypernatremia have an effect on the brainstem auditory evoked potential (BAEP) for three species of anurans, Bufo americanus, Bufo terrestris and Rana pipiens. I used the BAEP to monitor auditory and nervous system function as dehydration and hypernatremic levels were increased. Since anurans can be exposed to increased plasma and CSF sodium concentrations relative to mammals, and the EEG for the mammals begins to show signs of stress at a sodium concentration of $200 \mathrm{mM}$, the BAEP should be able to reveal changes in the anuran nervous system, if any occur, when the sodium ion concentration increases beyond the mammalian maximum.

\section{METHODS}

\section{Dehydration}

Before testing, each frog's bladder was emptied and body mass determined to $0.1 \mathrm{~g}$. Periodically, body weight and cloacal temperature were measured to $0.1^{\circ} \mathrm{C}$ (VWR digital thermometer 500 with rectal probe). Animals were allowed to dehydrate at room temperature $\left(22-25^{\circ} \mathrm{C}\right)$ which yielded a dehydration rate of one to two percent body-weight loss per hour. At intervals of two to three hours, the animals were removed from cages, BAEPs measured at $87 \mathrm{~dB}$ SPL and $78 \mathrm{~dB}$ SPL, threshold SPL, and weight and temperature recorded. This was repeated until the animals had reached 
a "critical activity point" (CAP), the dehydration level that rendered the animal incapable of performing a righting reflex within 10 seconds (Hillman, 1980). It usually takes an anuran one second to right themselves.

\section{Hypernatremia}

When deprived of their bladder water store, one can accurately predict the plasma concentrations of most solutes in a dehydrating anuran (Shoemaker, 1964). Alternatively, the salt concentration effect of dehydration can be mimicked by saline injection. To increase plasma $\mathrm{Na}^{+}$ concentrations to dehydration percentages, frogs were loaded with $\mathrm{NaCl}$ by injecting into the dorsal lymph sac a $2 \mathrm{M}$ solution of $\mathrm{NaCl}$. The $\mathrm{NaCl}$ injected was equal to the amount necessary to increase the $\mathrm{Na}^{+}$concentration to the levels found in a dehydrating frog at a given water loss percentage. The water loss percentages selected were: $20 \%, 25 \%, 30 \%$ and $35 \%$ for $R$. pipiens and $20 \%, 30 \%, 38 \%$ and $42 \%$ for the bufonids. The $\mathrm{mM}$ amount of $\mathrm{NaCl}$ necessary was calculated by the formula $C_{f}=C_{i}\left(V_{i} / V_{f}\right)$, where $V_{i}=$ initial water content $(80 \%) \mathrm{m}, \mathrm{V}_{\mathrm{f}}=$ final water content ( $80 \%-\%$ body mass lost), $\mathrm{C}_{\mathrm{i}}=$ initial $\mathrm{Na}+$ concentration, and $\mathrm{C}_{\mathrm{f}}=$ final $\mathrm{Na}+$ concentration (Shoemaker, 1964; Hillman, 1988). After injection, the frogs were allowed to equilibrate for six hours before BAEP recording. A sample of dorsal lymph sac fluid or of blood from a catheter inserted in the abdominal vein was obtained after each equilibration period and the $\mathrm{Na}^{+}$concentration determined using flame photometry (Instrumentation Laboratory 143), to verify the salt load and corresponding water loss percentage. 


\section{RESULTS}

\section{Dehydration}

BAEPs were recorded from 12 Bufo americanus and 17 Rana pipiens that were allowed to dehydrate to a "critical activity point" (CAP) at an average rate of $1 \%$ body-weight loss per hour. The level of dehydration was determined as the percent of body-weight lost (100\% - (current bodyweight)/(original body-weight)). The average CAP was $42.6 \pm 4.18$ (95\% confidence)\% for B. americanus and $38.7 \pm 3.78 \%$ for $R$. pipiens. Some of the anurans were found to have surpassed their CAP. These animals, when BAEPs were recorded, were able to right themselves in 3 - 5 seconds, which was longer than the usual one second but shorter than the criterium for CAP. These animals were allowed to dehydrate further to reach CAP, but later analysis of the BAEPs showed that an extension of the righting reflex to 3 - 5 seconds was sufficient to indicate that CAP had been reached in these species.

Figure 19 shows the effect of dehydration on the waveform morphology of the BAEP for $R$. pipiens.. The BAEP was unaffected by the decrease in water content for the frogs up to the CAP. Once CAP was reached, the overall BAEP began to decrease in amplitude and the breadth of the individual peaks increased. The individual whose data were selected for figure 19 had been allowed to dehydrate past its CAP (as defined above).

Dehydration had a similar effect on the waveform morphology of the BAEP for B. americanus as seen for the frogs (Figure 20). The BAEP was unchanged at all dehydration percentages below the CAP. At CAP, the overall BAEP decreased in amplitude and increased in peak breadth. 

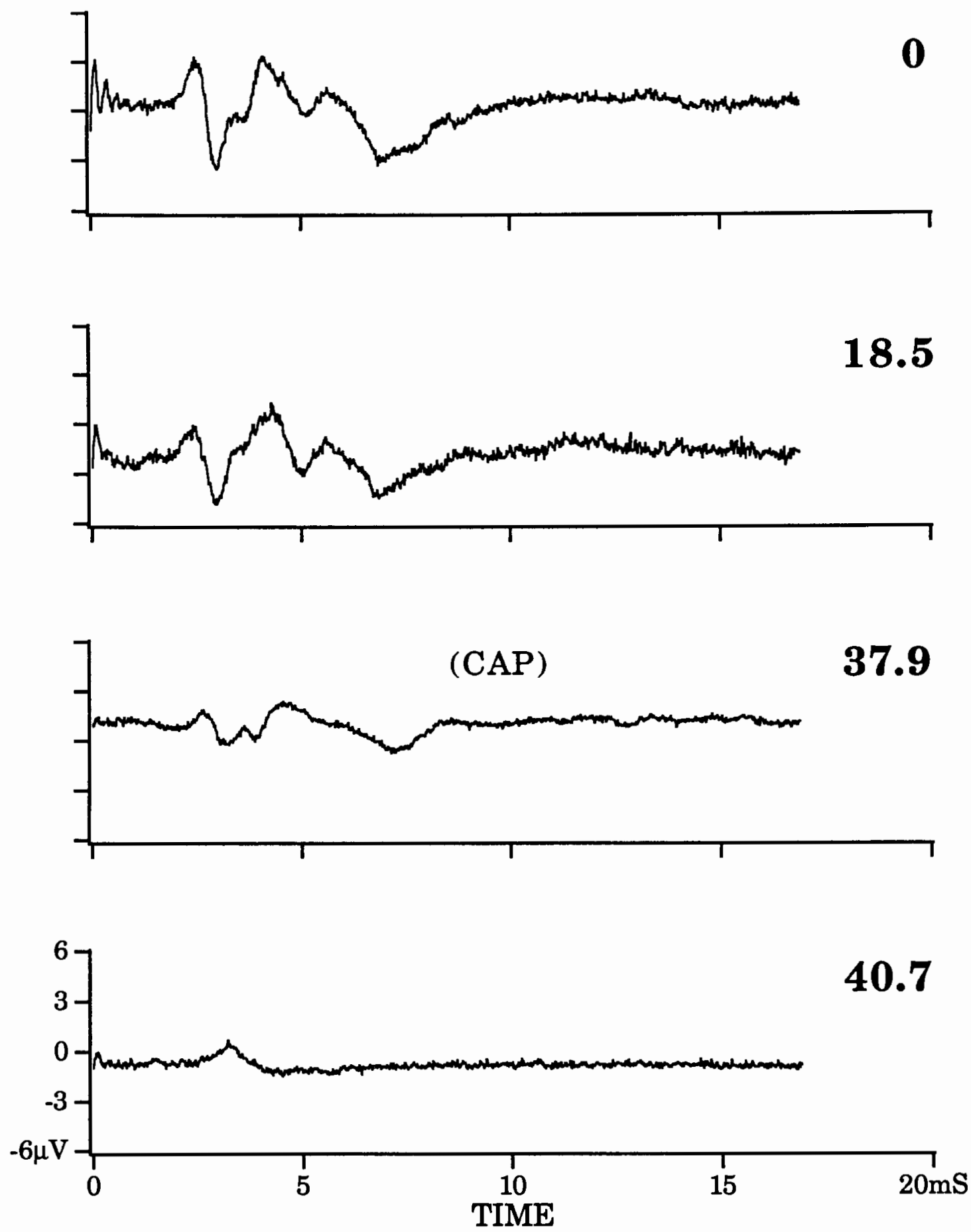

Figure 19. Effect of dehydration on the BAEP of one Rana pipiens. BAEPs were recorded at four different levels of dehydration, in response to an acoustic stimulus delivered at a sound level of $87 \mathrm{~dB}$ SPL. 
DEHYDRATION

(\% body mass loss)

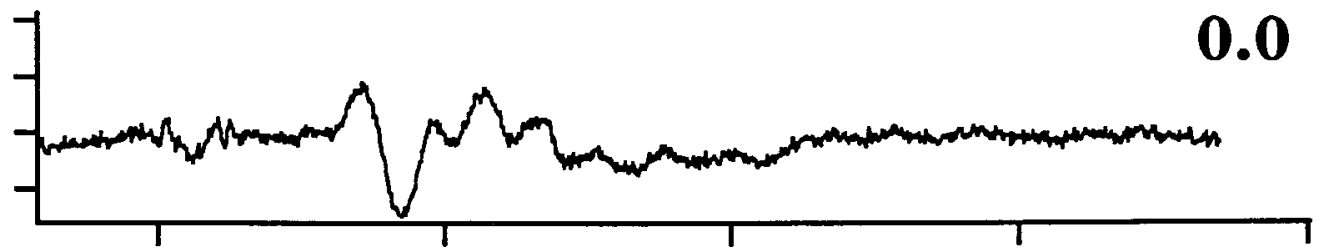

10.9
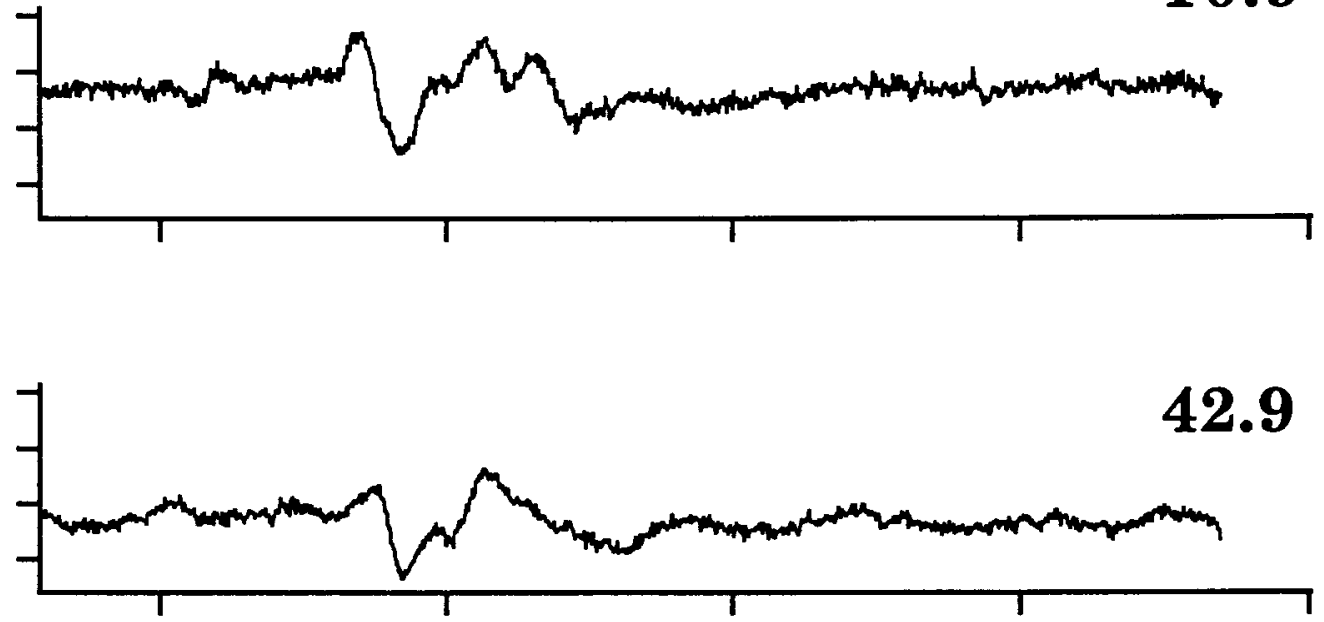

45.1
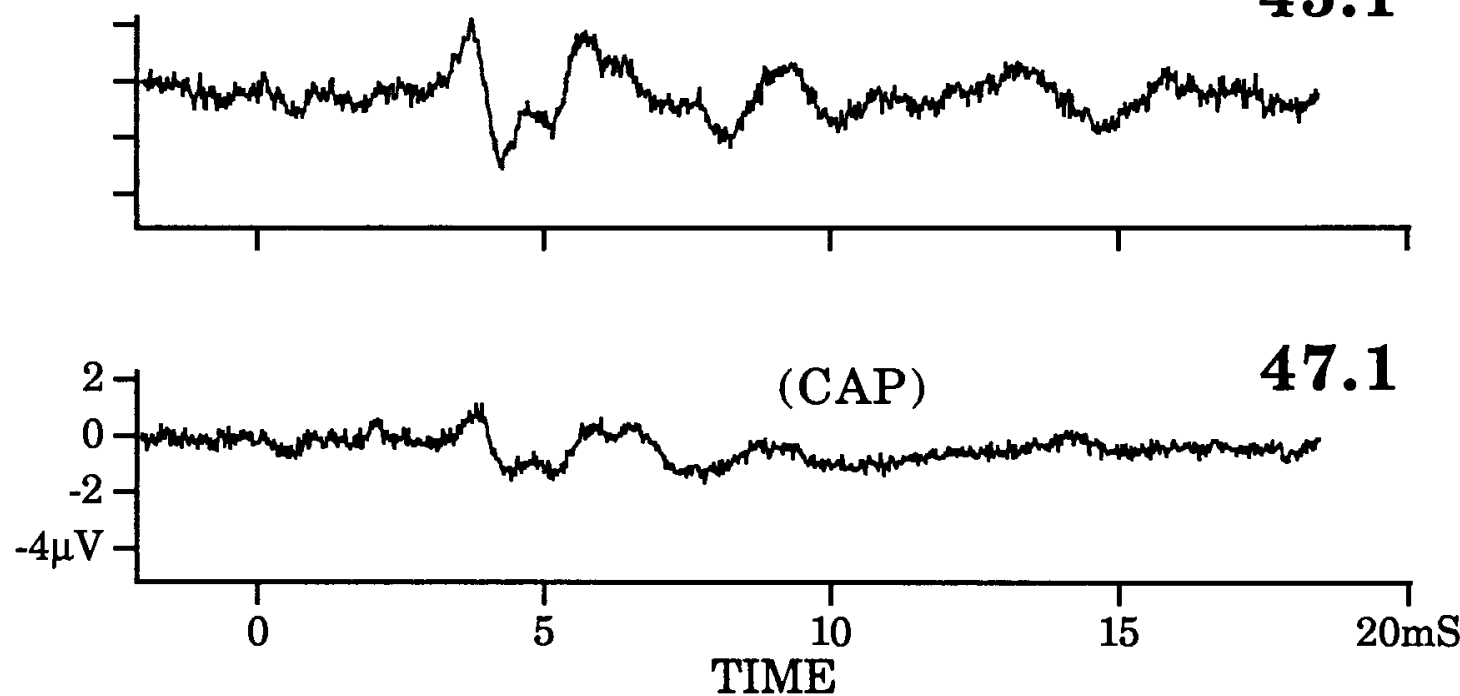

Figure 20. Effect of dehydration on the BAEP of one Bufo americanus. BAEPs were recorded at five different levels of dehydration, in response to an acoustic stimulus delivered at an sound level of $87 \mathrm{~dB}$ SPL. 
BAEP threshold was stable for both species at less-than-CAP dehydration with BAEP threshold for $R$. pipiens lower than the $B$. americanus BAEP threshold, as expected (see Chapter I) (Figure 21). Once CAP was reached, BAEP threshold for $R$. pipiens increased, while BAEP threshold for $B$. americanus remained unchanged, despite changes in waveform morphology.

For both species of amphibians, the peak I latency and interpeak latencies were unchanged at dehydration percentages less than CAP dehydration (Figure 22). The only apparent difference between the species was for I-II interpeak latency (Figure 22B). For $R$. pipiens, as the dehydration percentage was increased past the CAP, peak I and the three interpeak latencies all increased markedly (Figure 22A-D). For $B$. americanus, once the dehydration percentage surpassed the CAP, only the III-IV interpeak latency had significantly increased (Mann-Whitney U test, $\mathrm{p}<.01$ ) (Figure 22D). The other latency measurements were unchanged.

The mean amplitudes of peaks I and IV for $R$. pipiens and $B$. americanus responded differently to the increase in dehydration percentage (Figure 23). For $R$. pipiens, the mean amplitudes of peaks I and IV remained stable and greater than the amplitude of peaks I and IV for B. americanus during dehydration levels below CAP (Figure 23A and B). Once CAP was reached, both peak amplitudes for $R$. pipiens decreased markedly to the peak amplitudes seen in B. americanus.. For B. americanus, both peak amplitudes remained stable throughout all dehydration percentages.

No improvement in error could be realized by normalizing data to percent of maximum amplitude, thus a plot of percent of maximum is not given for either species. Because of this large error throughout the entire 

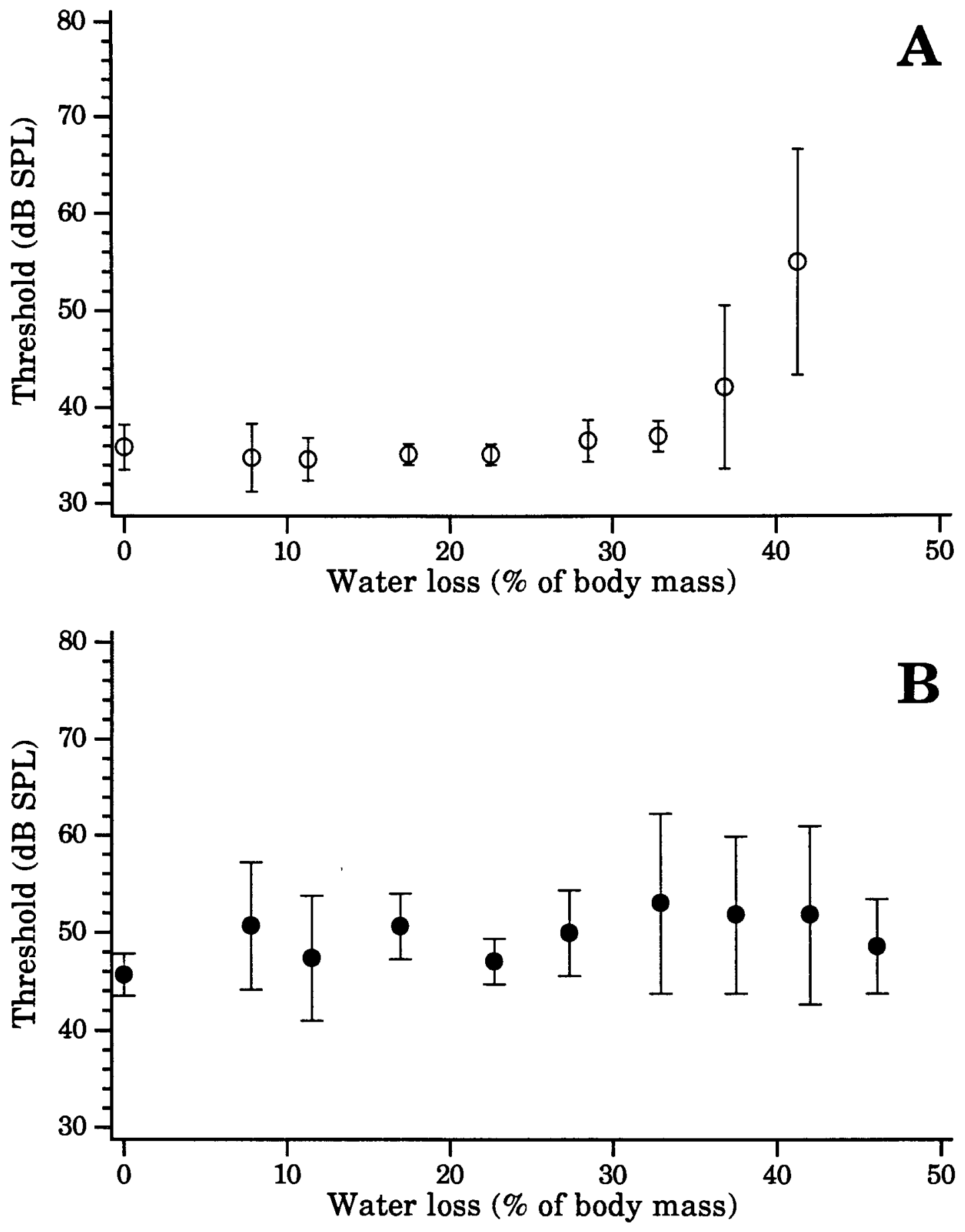

Figure 21. Mean BAEP threshold and 95\% confidence intervals in response to changes in dehydration percentage for Rana pipiens (A) and Bufo americanus (B). 

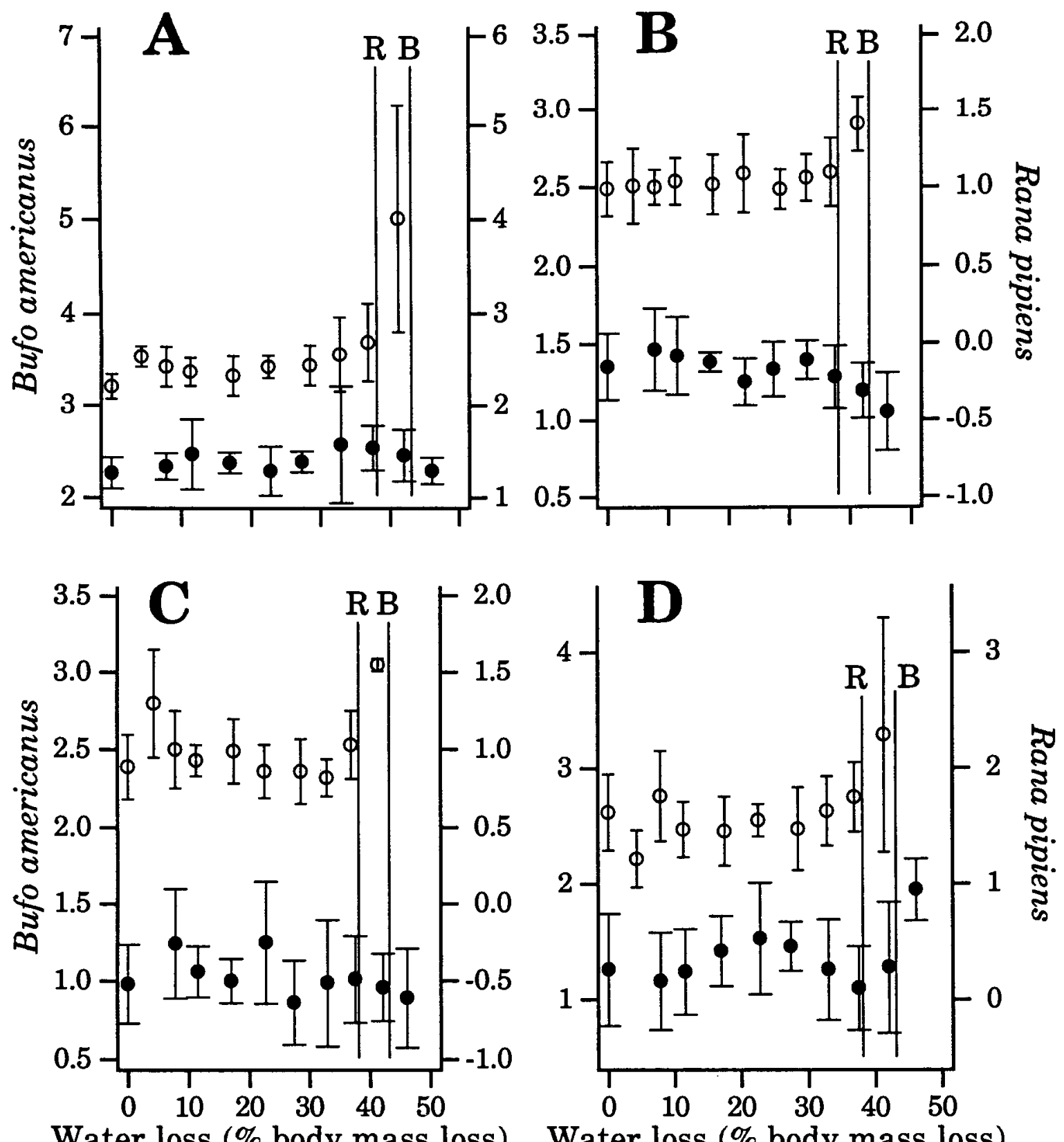

Water loss (\% body mass loss) Water loss (\% body mass loss)

Figure 22. A comparison of peak I latency and I-II, II-III and III-IV interpeak latencies to increased water loss for Rana pipiens and Bufo americanus. Unfilled circles; Rana pipiens. Filled circles; Bufo americanus. Points are means ( $\pm 95 \%$ confidence intervals). (A) Peak I latencies for both species in response to increased water loss. (B) I-II interpeak latencies for both species in response to increased water loss. (C) II-III interpeak latencies for both species in response to increased water loss. (D) III-IV interpeak latencies for both species in response to increased water loss. Note: Mean CAPs are labeled for each species ( $\mathrm{R}=$ Rana pipiens and $\mathrm{B}=$ Bufo americanus $)$. 

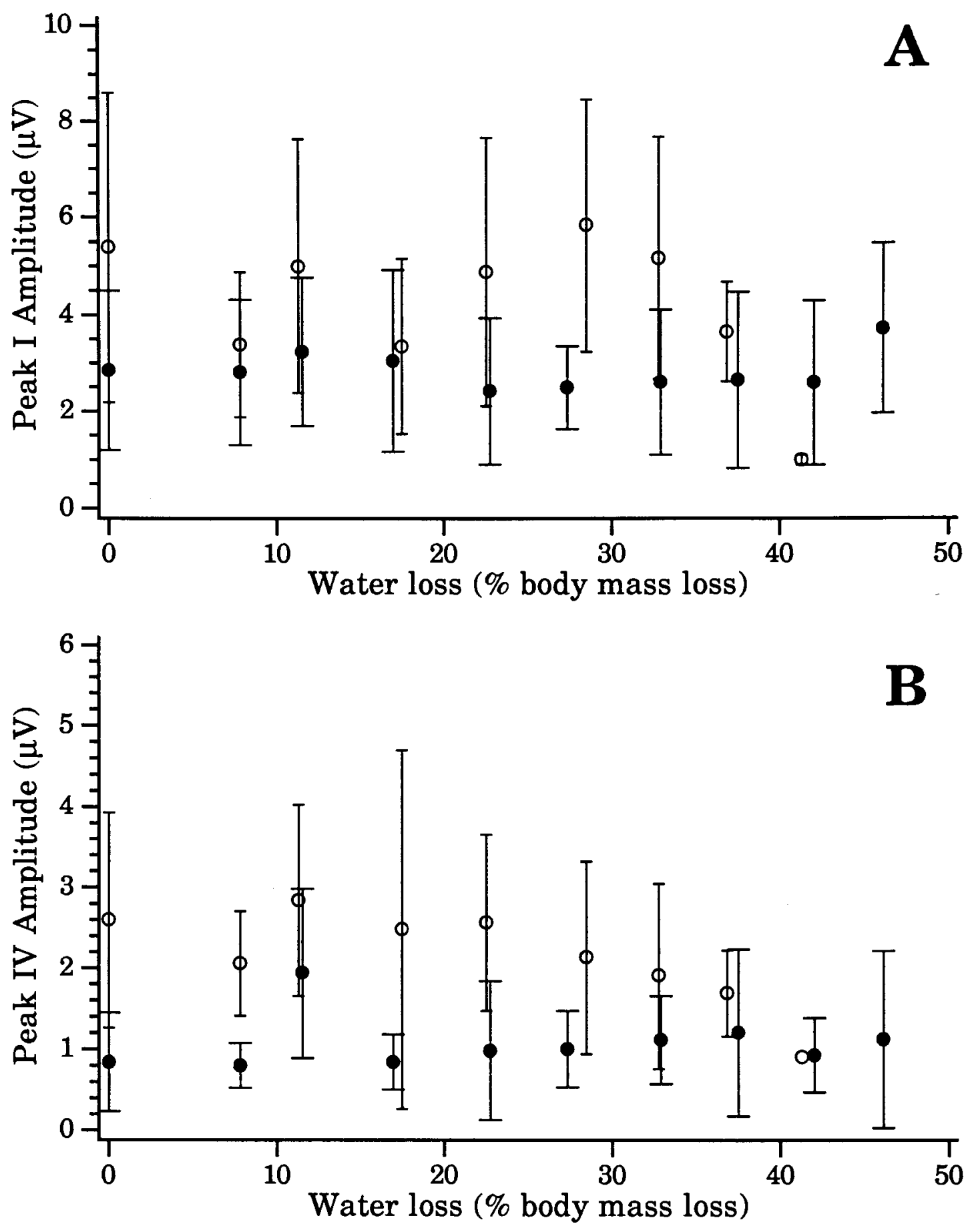

Figure 23. A comparison of the peak I (A) and IV (B) mean amplitudes for Rana pipiens and Bufo americanus to increases in the dehydration percentage. Unfilled circles Rana pipiens. Filled circles; Bufo americanus. Points are means $( \pm 95 \%$ confidence intervals) 
dehydration range, there does not seem to be a dehydration percentage in which either peaks will most likely be at their maximum for either species.

\section{Hypernatremia}

BAEPs were recorded from 4 Bufo americanus, 11 Bufo terrestris and 13 Rana pipiens that were injected with $2 \mathrm{M} \mathrm{NaCl}$ at the rate described in the methods, to increase the plasma sodium ion concentration (SL). Only three $B$. americanus, three $B$. terrestris and one $R$. pipiens reached their CAP.

For $R$. pipiens and B. americanus, the increase in salt concentration had no effect on the waveform morphology at values lower than CAP hypernatremia for either anuran. Once CAP was reached, the BAEP was reduced in amplitude in response to the critical sodium levels for all species tested.

The latencies of the peripheral peaks (I and II) changed with increasing SL, while the more central interpeak latencies (III and IV) remain unchanged. Peak I and I-II interpeak latencies initially decreased with increased SL of $20 \%$, but increased in latency with further increase in SL. This initial decrease could be a result of a increase in the body temperature of the animals during equilibration.

Peak I and IV amplitudes for the bufonids were unchanged in response to increase SL. Conversely, peak I and IV amplitudes decreased with increasing SL for $R$. pipiens. As a result, BAEP threshold was unchanged for the bufonids and increased for $R$. pipiens as the SL was increased.

All BAEP parameters measured exhibited a large range of variability. The trends described are general at best and further experiments are necessary before conclusions may be reached. 


\section{DISCUSSION}

For both the ranids and the bufonids, BAEPs were unaffected by increased levels of dehydration until the CAP was reached. This is in agreement with other studies, measuring EEGs in mammals (Arieff and Guisado, 1976; Dodge, et al., 1962). It is interesting that although the basic functioning of the nervous system is the same in all vertebrates, the CAP or tolerance to dehydration/hypernatremia is different. This would suggest that the failure of the nervous system at lethal levels of dehydration/ hypernatremia is not solely a function of the increased sodium concentration in the nervous system, but is a result of the hypertonic effects throughout many physiological systems. Changes in other systems will, of course impact nervous system function, e.g., decreased fluid volume in the circulatory system (Arieff, et al., 1979; Hillman, 1980).

The differential effects of dehydration on the BAEP are consistent with the ecology of $R$. pipiens and B. americanus. $R$. pipiens is usually found in or near wet surroundings (Tracy, 1976), while $B$. americanus are more terrestrial. Since B. americanus has an increased CAP relative to $R$. pipiens, it should be able to tolerate the decreased availability of water found in a more terrestrial environment better than $R$. pipiens.

There were very few animals which reached CAP for the hypernatremia study. This may be because of the rate of increase for sodium concentration. For all species, I attempted to increase the sodium concentration from $110 \mathrm{mM}$ to $147 \mathrm{mM}$, which is a $20 \%$ dehydration equivalent, in one step allowing equilibration over six hours. Less than half of the $R$. pipiens but all of the bufonids survived this initial increase. Other studies have reported high mortality rates when the sodium ion 
concentration is increased too quickly (Arieff and Guisado, 1976; Sotos, et al., 1960). At least the differential species mortality which I observed is certainly consistent with the notion that $B$. americanus and $B$. terrestris are more tolerant to hypernatremia than $R$. pipiens (Hillman, 1980).

All of the results from this study are preliminary and need to be repeated with more animals and with more gradual saline injection. Furthermore, using larger animals such as Bufo marinus and Rana catesbeiana would make it easier to draw fluid samples for measurement of $\mathrm{Na}^{+}$concentration and to maintain fluid volume. 


\section{CHAPTER IV}

\section{GENERAL DISCUSSION}

The BAEP is an excellent technique which allows long term monitoring of the anuran auditory system. One can use this technique to record a number of BAEPs from a single individual over many days with little threshold or latency measurement error (Table II).

There are a number of studies which could take advantage of this technique. An example would be a follow-up on the temperature experiments completed in this study. It would involve repeating the temperature experiment using a toad of similar size to Rana pipiens to compare the effects of temperature on a frog BAEP versus a toad BAEP. Toads respond differently to changes in temperature compared to frogs and operate at peak efficiency at a different temperature range (Putnam and Bennett, 1981) . These differences may be detected using the BAEP.

There are some changes in my BAEP recording technique which could reduce the signal-to-noise ratio in future studies. Currently, the anuran is connected to the common input of the differential amplifier via the copper manifold on which the anuran is positioned. To increase the effectiveness of the common mode rejection and decrease the contact variability with the ground connection, I would replace the reference electrode, placed in the dorsal lymphatic sac, with the common electrode and attach the reference electrode elsewhere on the cranium of the frog. The exact position of the 
reference electrode would need to be determined, so that all peaks of the BAEP are prominent in the waveform.

In conclusion, the BAEP is a helpful tool in the analysis of the auditory function in the anurans. 


\section{REFERENCES}

Achor, L. J. and A. Starr (1980) Auditory brain stem responses in the cat. I. intracranial and extracranial recordings. Electoencephalography and clinical neurophysiology 48:154-173.

Alvarado, R. H. (1972) The effects of dehydration on water and electrolytes in ambystoma tigrinum. Physiol. Zool. 45:43-53.

Andrew, R. D. (1991) Seizure and acute osmotic change: clinical and neurophysiological aspects. Journal of the Neurological Sciences 101:718.

Arieff, A. I. and R. Guisado (1976) Effects on the central nervous system of hypernatremic and hyponatremic states. Kidney International 10:104116.

Arieff, A. I., R. Guisado and V. C. Lazarowitz (1979) Pathophysiology of Hyperosmolar States. In: Disturbances in Body Fluid Osmolality, Andreoli, T. E., J. J. Grantham and F. C. R. Jr. (Ed.), American Physiological Society, Bethesda, 227-250.

Attias, J., et al. (1991) The effect of moderate heat stress on auditory brain stem evoked response in man. J. Therm. Biol. 16:249-253.

Bekesy, G. v. (1960) Experiments in Hearing. McGraw-Hill Book Company, New York.

Bishop, L. G. and M. S. Gordon (1967) Thermal adaptation of metabolism in anuran amphibians. In: Molecular Mechanisms of Temperature Adaptation, American Association for the Advancement of Science, 263-280.

Blair, W. F. (1958) Mating call in the speciation of anuran amphibians. The American Naturalist 92:27-50.

Borg, E. and L. Lofqvist (1982) Auditory brainstem response (ABR) to rarefaction and condensation clicks in normal and abnormal ears. Scand. Audiol. 11:227-235.

Brzoska, J., W. Walkowiak and H. Schneider (1977) Acoustic communication in the grass frog (Rana t. temporaria L.): calls, auditory thresholds and behavioral responses. J Comp Physiol A 118:173-186. 
Campbell, H. W. (1969) The effects of temperature on the auditory sensitivity of lizards. Physiol. Zool. 42:183-210.

Capranica, R. R. (1976) Morphology and physiology of the auditory system. In: Frog Neurobiology, Llinas, R. and W. Precht (Ed.), SpringerVerlag, Berlin, 551-575.

Corey, D. P. and A. J. Hudspeth (1983) Kinetics of the receptor current in the bullfrog saccular hair cells. J. Neurosci. 3:962-976.

Corso, J. F. (1963) Bone conducted thresholds for sonic and ultrasonic frequencies. J. Acoust. Soc. Am. 35:1738-43.

Corwin, J. T., T. H. Bullock and J. Schweitzer (1982) The auditory brain stem response in five vertebrate classes. Electoencephalography and clinical neurophysiology 54:629-641.

Counter, S. A. (1985) Brain-stem evoked potentials and noise effects in seagulls. Comparative Biochemistry 81A:837-845.

Covey, E., M. Vater and J. H. Casseday (1991) Binaural properties of single units in the superior olivary complex of the mustached bat. Journal of Neurophysiology 66:1080-1094.

Dodge, P. R., et al. (1962) Neurophysiologic disturbances in hypertonic dehydration. Trans. Am. Neurol. Assoc. 87:33-36.

Don, M., A. R. Allen and A. Starr (1977) Effect of click rate on the latency of auditory brain stem responses in humans. Ann. Otol. 86:186-195.

Don, M. and J. J. Eggermont (1978) Analysis of the click-evoked brainstem potentials in man using high-pass noise masking. J. Acoust. Soc. Am. 63:1084-1092.

Durrant, J. D. (1988) The Short Latency Auditory Evoked Potentials. American Speech-Language-Hearing Association, June, 1987.

Eatock, R. A. and G. A. Manley (1981) Auditory Nerve fibre activity in the tokay gecko. II. temperature effect on tuning. J Comp Physiol A 142:219-226.

Elberling, C. and M. Don (1987) Threshold characteristics of the human auditory brain stem response. Journal of the Acoustical Society of America 81:115-121. 
Feng, A. S. (1991) Differential innervation patterns of three divisions of frog auditory midbrain (Torus semicircularis). Journal of Comparative Neurology 306:613-630.

Feng, A. S., J. C. Hall and S. Siddique (1991) Coding of temporal parameters of complex sounds by frog auditory nerve fibers. Journal of Neurophysiology 65:424-445.

Feng, A. S., P. M. Narins and R. R. Capranica (1975) Three populations of primary auditory fibers in the bullfrog (Rana catesbeiana): their peripheral origins and frequency sensitivities. J. Comp. Physiol. 100:221-229.

Freeman, S., H. Sohmer and S. Silver (1991) The effect of stimulus repetition rate on the diagnostic efficacy of the auditory nerve-brain-stem evoked response. Electoencephalography and clinical neurophysiology 78:284290.

Frishkopf, L. S., R. R. Capranica and J. M. H. Goldstein (1968) Neural coding in the bullfrog's auditory system - a teleological approach. Proceedings of the IEEE 56:969-980.

Fuzessery, Z. M. and A. S. Feng (1983) Frequency selectivity in the anuran medulla: excitatory and inhibitory tuning properties of single neurons in the dorsal medullary and superior olivary nuclei. J Comp Physiol A 150:107-119.

Gage, P. W. (1976) Generation of end-plate potentials. Physiol. Rev. 56:177247.

Gatten, R. E. J. (1987) Activity metabolism of anuran amphibians: tolerance to dehydration. Physiol. Zool. 60:576-585.

Gerhardt, H. C. (1974) The significance of some spectral features in mating call recognition in the green treefrog (Hyla cinerea). J. Exp. Biol 61:229-241.

Gerhardt, H. C. (1978) Temperature coupling in the vocal communication system of the gray tree frog, Hyla versicolor. Sci. 199:992-994.

Gerhardt, H. C. and J. A. Doherty (1988) Acoustic communication in the gray treefrog, Hyla versicolor: evolutionary and neurobiological implications. J Comp Physiol A 162:261-278.

Gerhardt, H. C. and K. M. Mudry (1980) Temperature effects on frequency preferences and mating call frequencies in the green treefrog, $H y l a$ cinerea (Anura: Hylidae). Journal of Comparative Physiology. A 137:16. 
Glattke, T. J. (1983) Short-Latency Auditory Evoked Potentials:

Fundamental Bases and Clinical Applications. PRO-ED, Austin.

Gorga, M. P., P. J. Abbas and D. W. Worthington (1985) Stimulus Calibration in ABR Measurements. In: The Auditory Brainstem Response, Jacobson, J. T. (Ed.), College-Hill Press, Boston, 49-52.

Hall, J. C. and A. S. Feng (1991) Temporal processing in the dorsal medullary nucleus of the northern leopard frog (Rana pipiens pipiens). Journal of Neurophysiology 66:955-973.

Hartenstein, R. (1972) Principles of Physiology. Van Nostrand Reinhold, New York.

Hillery, C. M. and P. M. Narins (1984) Neurophysiological evidence for a traveling wave in the amphibian inner ear. Sci. 225:1037-1039.

Hillman, S. S. (1978) Some effects of dehydration on internal distributions of water and solutes in Xenopus laevis. Comp. Biochem. Physiol. 61A:303-307.

Hillman, S. S. (1980) Physiological correlates of differential dehydration tolerance in anuran amphibians. Copeia 1980:125-129.

Hillman, S. S. (1982) The effects of in vivo and in vitro hyperosmolality on skeletal muscle performance in the amphibians Rana pipiens and Scaphiopus couchii. Comp. Biochem. Physiol. 73A:709-712.

Hillman, S. S. (1987) Dehydration effects on cardiovascular and metabolic capacity in two amphibians. Physiol. Zool. 60:608-613.

Hillman, S. S. (1988) Dehydrational effects on brain and cerebrospinal fluid electrolytes in two amphibians. Physiol. Zool. 61:254-259.

Hubl, L., R. Mohneke and H. Schneider (1977) Temperature dependence of auditory thresholds in two central european anurans, Bombina variegata (L.) and Rana ridibunda ridibunda Pall. (amphibia), and its relation to calling. Behavioural Processes 2:305-314.

Hubl, L. and H. Schneider (1979a) Temperature and auditory thresholds: bioacoustic studies of the frogs Rana r. ridibunda, Hyla a. arborea and Hyla a savignyi (Anura, Amphibia). J Comp Physiol A 130:17-27.

Hubl, L. and H. Schneider (1979b) Temperature and auditory thresholds: bioacoustic studies of the frogs Rana r. ridibunda, Hyla a. arborea and Hyla a. savignyi (Anura, Amphibia). J Comp Physiol A 130:17-27. 
Huey, R. B. (1982) Temperature, physiology, and the ecology of reptiles. In: Biology of the Reptilia, Vol. 12, Physiological Ecology. Pough, F. H. (Ed.), Academic Press, London, 26-90.

Jacobson, J. T. (1985) An Overview of the Auditory Brainstem Response. In: The Auditory Brainstem Response, Jacobson, J. T. (Ed.), College-Hill Press, Boston, 3-12.

Krakauer, T. (1970) Tolerance limits of the toad, Bufo marinus, in south Florida. Comp. Biochem. Physiol. 33:15-26.

Kuffler, S. W., J. G. Nichols and A. R. Martin (1984) From Neuron to Brain. Sinauer Associates, Sunderland, MA.

Lasky, R. E. and E. Yang (1986) Methods for determining auditory evoked brain-stem response thresholds in human newborns. Electoencephalography and clinical neurophysiology 65:276-281.

Lewis, E. R. (1984) On the frog amphibian papilla. Scanning Electron Microscopy 4:1899-1919.

Lewis, E. R., et al. (1982a) Inner ear: dye injection reveals peripheral origins of specific sensitivities. Sci 215:1641-1643.

Lewis, E. R., E. L. Leverenz and H. Koyama (1982b) The tonotopic organization of the bullfrog amphibian papilla, and auditory organ lacking a basilar membrane. J. Comp. Physiol. 145:437-445.

Lombard, E. and I. R. Straughan (1974) Functional aspects of anuran middle ear structures. J. Exp. Biol. 61:71-93.

Lombard, R. E., R. R. Fay and Y. L. Werner (1982) Underwater hearing in the frog, Rana catesbeiana. J. Exp. Biol. 91:57-71.

Macdonald, J. A. (1981) Temperature compensation in the peripheral nervous system: antarctic vs. temperate poikilotherms. J Comp Physiol A 142:411-418.

Marsh, R. R., H. Yamane and W. P. Potsic (1984) Auditory brain-stem response and temperature: relationship in guinea pig. Electoencephalography and clinical neurophysiology 57:289-293.

Megela-Simmons, A. (1988) Masking patterns in the bullfrog (Rana catesbeiana). I: behavioral effects. Journal of the Acoustical Society of America 83:1087-1092. 
Megela-Simmons, A., C. F. Moss and K. M. Daniel (1985) Behavioral audiograms of the bullfrog (Rana catesbeiana) and the green tree frog (Hyla cinerea). Journal of the Acoustical Society of America 78:12361244.

Mohneke, R. and H. Schneider (1979) Effect of temperature upon auditory thresholds in two anuran species, Bombina v. variegata and Alytes $o$. obstetricans (Amphibia, Discoglossidae). J Comp Physiol A 130:9-16.

Moller, A. R. and P. J. Janetta (1985) Neural Generators of the Auditory Brainstem Response. In: The Auditory Brainstem Response, Jacobson, J. T. (Ed.), College-Hill Press, Boston, 13-31.

Moller, A. R., P. Jannetta and M. B. Moller (1982) Intracranially recorded auditory nerve response in man. Archives of Otolaryngology 108:77-82.

Moore, J. K. (1987) The human auditory brain stem: a comparative view. Hearing Research 29:1-32.

Penna, M., et al. (1990) Midbrain auditory sensitivity in toads of the genus Bufo (Amphibia - Bufonidae) with different vocal repertoires. J Comp Physiol A 167:673-681.

Pettigrew, A. G., M. Anson and S. H. Chung (1981) Hearing in the frog: a neurophysiological study of the auditory response in the midbrain. Proc. R. Soc. Lond. B 212:430-457.

Putnam, R. W. and A. F. Bennett (1981) Thermal dependence of behavioural performance of anuran amphibians. Anim. Behav. 29:502-509.

Ronken, D. A. (1990) Basic properties of auditory-nerve responses from a 'simple' ear: the basilar papilla of the frog. Hearing Research 47:6382.

Rose, G. J., E. A. Brenowitz and R. R. Capranica (1985) Species specificity and temperature dependency of temporal processing by the auditory midbrain of two species of treefrogs. J Comp Physiol A 157:763-769.

Rosenblum, S. M., R. A. Ruth and T. J. Gal (1985) Brain stem auditory evoked potential monitoring during profound hypothermia and circulatory arrest. Ann Otol Rhinol Laryngol 94:

Schiotz, A. (1973) Evolution of anuran mating calls ecological aspects. In: Evolutionary Biology of the Anurans, Vial, J. L. (Ed.), Univ. Missouri Press, 311-319. 
Schwartz, D. M. and G. A. Berry (1985) Normative Aspects of the ABR. In: The Auditory Brainstem Response, Jacobson, J. T. (Ed.), College-Hill Press, Boston, 65-97.

Seaman, R. L. (1991) Method to record evoked potentials from the frog eighth nerve. Hearing Research 51:301-306.

Shoemaker, V. H. (1964) The effects of dehydration on electrolyte concentrations in a toad, Bufo marinus. Comp. Biochem. Physiol. 13:261-271.

Smith, V. D. E. and C. M. Jackson (1931) The changes during desiccation and rehydration in the body and orgas of the leopard frog (Rana pipiens). Biol. Bull. 60:80-93.

Sohmer, H., S. Freeman and M. Schmuel (1989) ABR threshold is a function of blood oxygen level. Hearing Research 40:87-92.

Sotos, J. F., et al. (1960) Studies in experimental hypertonicity I. pathogenesis of the clinical syndrome, biochemical abnormalities and cause of death. Pediatrics 26:925-938.

Star, R. A. (1990) Southwestern internal medicine conference: hyperosmolar states. Am. J. Med. Sci. 300:402-412.

Strain, G. M., et al. (1987) Brain-stem auditory evoked potentials in the alligator. effects of temperature and hypoxia. Electoencephalography and clinical neurophysiology 67:68-76.

Straughan, I. A. (1973) Evolution of anuran mating calls bioacoustical aspects. In: Evolutionary Biology of the Anurans, Vol. Vial, J. L. (Ed.), Univ. Missouri Press, 321-327.

Thorson, T. and A. Svihla (1943) Correlation of the habitats of amphibians with their ability to survive the loss of body water. Ecology 24:374381.

Thorson, T. B. (1955) The relationship of water economy to terrestrialism in amphibians. Ecology 36:100-116.

Tracy, C. R. (1976) A model of the dynamic exchanges of water and energy between a terrestrial amphibian and its environment. Ecological Monographs 46:293-326.

van Dijk, P., E. R. Lewis and H. P. Wit (1990) Temperature effects on auditory nerve fiber response in the american bullfrog. Hearing Research 44:231-240. 
van Olphen, A. F., M. Rodenburg and C. Verwey (1979) Influence of the stimulus repetition rate on brain-stem-evoked responses in man. Audiology 18:388-394.

van Stokkum, I. H. M. and C. C. A. M. Gielen (1989) A model for the peripheral auditory nervous system of the grassfrog. Hearing Research 41:71-86.

Werner, Y. L. (1976) Optimal temperatures for inner-ear performance in gekkonid lizards. J. Exp. Zool. 195:319-352.

Wilczynski, W. and R. R. Capranica (1984) The auditory system of anuran amphibians. Progress in Neurobiology 22:1-38.

Wilczynski, W., H. H. Zakon and E. A. Brenowitz (1984) Acoustic communication in spring peepers: call characteristics and neurophysiological aspects. Journal of Comparative Physiology. A 155:577-584.

Yost, W. A. and D. W. Nielsen (1985) Fundamentals of Hearing: An Introduction. CBS College Publishing, New York.

Zakon, H. H. (1983) Reorganization of connectivity in amphibian central auditory system following VIIIth nerve regeneration: time course. 49:1410-1427.

Zelick, R. and P. M. Narins (1985) Temporary threshold shift, adaptation, and recovery characteristics of frog auditory nerve fibers. Hearing Research 17:161-176.

Zweifel, R. G. (1968) Effects of temperature, body size, and hybridization on mating calls of toads, Bufo a. americanus and Bufo woodhousii fowleri. Copeia 269-285. 